\title{
Potentiation of Divergent Medial Amygdala Pathways Drives Experience-Dependent Aggression Escalation
}

\author{
${ }^{\circledR}$ Jacob C. Nordman, ${ }^{1,2}{ }^{\oplus}$ Xiaoyu Ma, ${ }^{1}{ }^{\circledR}$ Qinhua Gu, ${ }^{1}$ Michael Potegal, ${ }^{3}{ }^{\circledR} \mathrm{He}$ Li, ${ }^{4}{ }^{\circledR}$ Alexxai V. Kravitz, ${ }^{5}$ and \\ (-) Zheng Li ${ }^{1}$ \\ ${ }^{1}$ Section on Synapse Development and Plasticity, National Institute of Mental Health, National Institutes of Health, Bethesda, Maryland 20892, \\ ${ }^{2}$ National Institute of General Medical Sciences, National Institutes of Health, Bethesda, Maryland 20892, ${ }^{3}$ Program in Occupational Therapy, \\ Center for Neurobehavioral Development, University of Minnesota, Minneapolis, Minnesota 55455, ${ }^{4}$ Department of Psychiatry, Uniformed Services \\ University, Bethesda, Maryland 20892, and ${ }^{5}$ Eating and Addiction Section, Diabetes, Endocrinology, and Obesity Branch, National Institute of \\ Diabetes and Digestive and Kidney Diseases, National Institutes of Health, Bethesda, Maryland 20892
}

Heightened aggression can be serious concerns for the individual and society at large and are symptoms of many psychiatric illnesses, such as post-traumatic stress disorder. The circuit and synaptic mechanisms underlying experience-induced aggression increase, however, are poorly understood. Here we find that prior attack experience leading to an increase in aggressive behavior, known as aggression priming, activates neurons within the posterior ventral segment of the medial amygdala (MeApv). Optogenetic stimulation of MeApv using a synaptic depression protocol suppresses aggression priming, whereas high-frequency stimulation enhances aggression, mimicking attack experience. Interrogation of the underlying neural circuitry revealed that the MeApv mediates aggression priming via synaptic connections with the ventromedial hypothalamus (VmH) and bed nucleus of the stria terminalis (BNST). These pathways undergo NMDAR-dependent synaptic potentiation after attack. Furthermore, we find that the MeApv-VmH synapses selectively control attack duration, whereas the MeApv-BNST synapses modulate attack frequency, both with no effect on social behavior. Synaptic potentiation of the MeApv-VmH and MeApv-BNST pathways contributes to increased aggression induced by traumatic stress, and weakening synaptic transmission at these synapses blocks the effect of traumatic stress on aggression. These results reveal a circuit and synaptic basis for aggression modulation by experience that can be potentially leveraged toward clinical interventions.

Key words: aggression; attack experience; medial amygdala; synaptic plasticity; traumatic stress

Significance Statement

Heightened aggression can have devastating social consequences and may be associated with psychiatric disorders, such as post-traumatic stress disorder. The circuit and synaptic mechanisms underlying experience-induced aggression escalation, however, are poorly understood. Here we identify two aggression pathways between the posterior ventral segment of the medial amygdala and its downstream synaptic partners, the ventromedial hypothalamus and bed nucleus of the stria terminalis that undergo synaptic potentiation after attack and traumatic stress to enhance aggression. Notably, weakening synaptic transmission in these circuits blocks aggression priming, naturally occurring aggression, and traumatic stress-induced aggression increase. These results illustrate a circuit and synaptic basis of aggression modulation by experience, which can be potentially targeted for clinical interventions.

Received Feb. 16, 2020; revised Mar. 25, 2020; accepted Apr. 14, 2020

Author contributions: J.C.N., X.M., H.L., A.V.K., and Z.L. designed research; J.C.N. and Q.G. performed research; J.C.N., X.M., and Q.G. analyzed data; J.C.N. wrote the first draft of the paper; J.C.N., M.P., H.L., A.V.K., and Z.L. edited the paper; J.C.N. and Z.L. wrote the manuscript.

This work was supported by National Institute of Mental Health Intramural Research Program 1Z1AMH002881 to Z.L., and National Institute of General Medical Sciences Postdoctoral Research Associate Training Program to J.C.N. We thank Daniel Letchford, Lindsay Ejoh, Princess Miranda, and Winnie Gao for analysis of behavioral and immunohistochemical data.

The authors declare no competing financial interests.

Correspondence should be addressed to Zheng Li at lizheng2@mail.nih.gov.

https://doi.org/10.1523/JNEUROSCI.0370-20.2020

Copyright $(2020$ the authors

\section{Introduction}

Aggression is an adaptive behavior intended to protect one from harm, conserve resources, and establish dominance. Maladaptive aggressive behavior, however, can have unintended, destructive consequences. High aggressive arousal and recurrent aggressive outbursts occur in some psychiatric patients, and are included in the diagnostic criteria for a number of psychiatric conditions, including conduct disorder, intermittent explosive disorder, and post-traumatic stress disorder (PTSD) (American Psychiatric Association, 2013).

In taxa from insects to mammals, aggressive behavior is strongly influenced by social and sexual experience. For example, 
a single attack on, or even just a brief exposure to, a samesex conspecific primes a short-term escalation in aggression in rodents termed "aggression priming" (Largerspetz, 1969; Tellegen and Horn, 1972; Potegal and Popken, 1985; Potegal, 1992; Miczek et al., 2013). Prior winning experience enhances aggression and probability of winning future contests, and single housing and copulation can transform sexually naive, nonaggressive males into aggressive ones (Valzelli, 1973; Flannelly et al., 1982; Potegal, 1992; Hsu et al., 2006; Stevenson and Rillich, 2012; Miczek et al., 2013; Hoopfer, 2016). These observations suggest that experience induces physiological changes in the brain to modulate aggressive behavior. Despite the significant impact of experience on aggression, however, little is known about what brain regions, neural circuits, and physiological processes mediate experience-dependent escalation of aggressive behavior.

The medial amygdala (MeA) is a key node in the neuroanatomical pathway of aggression (Nelson and Trainor, 2007). The $\mathrm{MeA}$ is one of three main subdivisions of the amygdala, located within the anterior temporal lobe and conserved between rodents and humans. In humans, the MeA is a target for successful neurosurgical interventions to treat intractable escalated aggression (Mpakopoulou et al., 2008). In rodents, immunostaining of c-Fos, a marker for neuronal activation (Morgan et al., 1987), shows that the MeA is activated during social behaviors, such as investigation, mating, and aggression (Erskine, 1993; Fernandez-Fewell and Meredith, 1994; Kollack-Walker and Newman, 1995, 1997; Heeb and Yahr, 1996; Choi et al., 2005; Lin et al., 2011; Kim et al., 2015).

The MeA can be parcellated into anteroventral, anterodorsal, posteroventral (MeApv), and posterodorsal (MeApd) parts, all of which are activated by conspecific fighting (Kollack-Walker and Newman, 1995; Lin et al., 2011). These subnuclei have different projection patterns and cell type compositions (Canteras et al., 1995). For example, projections from the MeApd to the ventromedial hypothalamic nucleus $(\mathrm{VmH})$, another key node in aggression, are largely GABAergic and segregated from those of the MeApv, which are mostly glutamatergic (Canteras et al., 1995; Choi et al., 2005). The functional dissection of the MeA in social behaviors, such as aggression, has recently been facilitated by genetic and optogenetic tools for cell type- and region-specific manipulation of neural activities. This line of research shows functional heterogeneity among different MeA cell types in aggressive behavior. Activation of GABAergic neurons in the MeApd and MeApv triggers attack and is required for ongoing aggressive behavior (Hong et al., 2014; Padilla et al., 2016). MeApv neurons containing the dopamine receptor Type 1 are a heterogeneous population containing both GABAergic and glutamatergic neurons that project onto the $\mathrm{VmH}$ and BNST to regulate aggression toward an intruder (Miller et al., 2019). GABAergic neurons expressing the neuropeptide Y Type 1 receptor in the MeApv, which receive input from $\mathrm{VmH}$ neurons and subsequently project onto the BNST, modulate territorial aggression during starvation (Padilla et al., 2016). Chemogenetic inhibition of aromatase-expressing neurons in the MeApd inhibits aggressive behavior (Unger et al., 2015). Optogenetic activation of glutamatergic neurons in the MeApd suppresses social interaction, including attack and sexual behavior (Hong et al., 2014). Despite these recent studies, it is unclear whether the $\mathrm{MeA}$ is involved in experience-dependent aggression escalation.

$\mathrm{MeA}$ neurons project to several areas that are activated by intermale aggression, such as the $\mathrm{VmH}$ and BNST, with the posterior segment of the MeA being the best characterized (Gomez and Newman, 1992; Canteras et al., 1995; Kollack-Walker and
Newman, 1995; Coolen and Wood, 1998; Haller et al., 2006; Nelson and Trainor, 2007; Pardo-Bellver et al., 2012; Golden et al., 2016). Neurons within the posterior MeA project to the ventrolateral ( $\mathrm{VmHvl})$, central, and dorsomedial regions of the $\mathrm{VmH}$ and the lateral and medial regions of the BNST (Canteras et al., 1995; Pardo-Bellver et al., 2012). Within the VmH, axons of the MeApd are more densely found in the $\mathrm{VmHvl}$, whereas axons of the MeApv are more evenly distributed throughout. Although the posterior MeA axons are found in both the lateral and medial regions of the BNST, they are most densely found in the medial BNST.

The $\mathrm{VmH}$ is the most thoroughly studied target area of the $\mathrm{MeA}$ in aggression regulation (Kruk, 2014). Electrical or optogenetic activation of the VmHvl induces attack, whereas optogenetic inhibition of this area suppresses attack (Kruk et al., 1983; Lammers et al., 1988; Lin et al., 2011; C. F. Yang et al., 2013; Lee et al., 2014). A subset of VmHvl neurons exhibit a maximum increase in firing rate during intermale attack and couples their firing activities to the imminence and intensity of future attacks (Falkner et al., 2014). Social experience shapes the response of estrogen receptor 1-expressing $\mathrm{VmHvl}$ neurons to conspecific sex and defense against an aggressive conspecific and the ability of progesterone receptor expressing $\mathrm{VmHvl}$ neurons to regulate offensive attack behavior against males (Remedios et al., 2017; T. Yang et al., 2017; Pan et al., 2020). Additionally, the VmHvl mediates foot shock-provoked attack behavior in socially isolated mice (Chang et al., 2015, 2018; Chang and Gean, 2019). In contrast to the $\mathrm{VmHvl}$, the dorsomedial region of the $\mathrm{VmH}$ has been associated with social fear (Silva et al., 2013; L. Wang et al., 2015).

The BNST is also activated by attack (Haller et al., 2006; Lin et al., 2011; Bayless et al., 2019). Activation of the posterior segment of the medial BNST increases social and attack behavior through the metabotropic glutamate receptor Type 7 (MasugiTokita et al., 2016). Aromatase-expressing neurons in the principle component of the medial BNST are required for intermale aggression (Bayless et al., 2019). Interestingly, repeated winning of fighting contests increases androgen receptor expression in the medial anterior aspect of the BNST (Fuxjager et al., 2010), suggesting a potential role for the medial BNST in experiencedependent aggression enhancement. A thorough examination of this possibility is warranted.

Aggression is commonly evaluated from attack behavior, which is comprised of a sequence of events. In rodents, it is preceded by approach and investigation (e.g., sniffing), followed by agonistic behaviors (e.g., lateral threat, chasing, biting and boxing) (Blanchard and Blanchard, 1977; Simon and Gandelman, 1981). It is unclear whether different stages of attack are individually controlled by separate brain regions or collectively by the same ones.

In this study, we examined the role of the MeApv in experience-dependent modification of aggression. We found that potentiation of synapses between MeApv neurons and their two target regions, the $\mathrm{VmH}$ and BNST, underlies aggression escalation induced by prior attack behavior and traumatic stress. Moreover, the MeApv-BNST and MeApv-VmH circuits influence different stages of attack: the MeApv-BNST synapses regulate attack initiation, whereas the MeApv-VmH synapses mainly act on the maintenance of attack. These findings indicate that the MeApv-BNST and MeApv-VmH circuits undergo agonistic and traumatic experience-dependent plasticity, and that these circuits and synaptic potentiation may be targeted to alleviate post-traumatic aggression escalation. 


\section{Methods and Materials \\ Animals}

All animal protocols were approved by the Animal Care and Use Committee. C57BL/6 mice were purchased from Charles River Laboratories and $v G l u t 2^{+}$mice were purchased from Jackson Laboratory and housed under a $12 \mathrm{~h}$ light (9:00 P.M. to 9:00 A.M.)/ dark (9:00 A.M. to 9:00 P.M.) cycle with ad libitum access to water and food. For experiments testing spontaneous aggressive behavior and involving foot shock, mice were individually housed for 3 weeks before testing. For experiments involving 5-100 Hz optogenetic stimulation, mice were group housed with 4 per cage before surgery and 2 per cage with a divider in between after surgery. The smaller, submissive target mice were group-housed with littermates. Male mice were used for all experiments.

\section{Surgery}

Six- to 7 -week-old C57BL/6 or $v$ Glut $2^{+}$male mice were anaesthetized with isoflurane (3\% for induction and $1 \%$ for maintenance) and then placed onto a stereotaxic frame (David Kopf Instruments). Bilateral craniotomy was made, and $500 \mathrm{nl}$ virus was injected into the MeApv (AP: $-1.50 \mathrm{~mm}$; ML, $\pm 2.1 \mathrm{~mm}$; DV, $-5.15 \mathrm{~mm}$ ) using a $5 \mu \mathrm{l}$ gas-tight Hamilton syringe (33gauge, beveled needle) at a rate of $0.1 \mu \mathrm{l} / \mathrm{min}$. After injection, the needle was left in place for an additional $5 \mathrm{~min}$ and then slowly withdrawn. After viral injection, ferrule-terminated optical fibers ( $100 \mu \mathrm{m}$ in diameter, ThorLabs) were placed $100 \mu \mathrm{m}$ above the viral injection site at the MeApv or at a midline position above the VmH (AP: 1.5 ; ML: $0 \mathrm{~mm}$; DV: -5.6 ), BNST (AP: $0.14 \mathrm{~mm}$; ML: $0 \mathrm{~mm}$; DV: $-3.8 \mathrm{~mm}$ ), medial preoptic nucleus (MPO) (AP: $0.26 \mathrm{~mm}$; ML: $0 \mathrm{~mm}$; DV: $-4.6 \mathrm{~mm}$ ), or lateral septum (LS) (AP: $0.38 \mathrm{~mm}$; ML: $0 \mathrm{~mm}$; DV: $-2.2 \mathrm{~mm}$ ). Optical fibers were secured to the skull using Metabond (Parkell), stainless-steel screws (PlasticsOne), and dental cement (DuraLay). Mice recovered for 3 weeks before MeApv stimulation or 8 weeks before stimulation of MeApv axons at the MeApv projection sites. For behavior experiments, $17.2 \%$ of operated animals were excluded from analysis due to viral mistargeting.

For optrode implantation, the optrode was lowered into the $\mathrm{VmH}$ or BNST at $100 \mu \mathrm{m}$ deeper than the above coordinates 6 weeks after viral injection. The craniotomy was sealed with bone wax. Two stainless-steel screws were inserted into the skull over the cerebellum and olfactory bulb to be used as ground and reference. The microdrive attached to an optrode was protected by a grounded copper mesh to block the external electrical noise. For in vivo recording experiments, $33.1 \%$ of operated animals were excluded from analysis due to either viral or optrode mistargeting.

\section{Behavioral tests and data analysis}

Aggression test. Mice were housed in groups of 2 with a perforated divider between them to test for the effect of high-frequency photostimulation (HFPS) on aggression, or individually housed for testing primed or naturally occurring aggression and for the traumatic stress experiment (Rau et al., 2005). All behavioral experiments took place during the dark cycle of the day. On the day of testing, mice were transferred to a behavioral room and allowed to acclimate for at least $1 \mathrm{~h}$. For aggression testing, mice were placed into a high-walled, novel cage and left to acclimate for an additional $20 \mathrm{~min}$. Younger, group-housed target males (conspecific) were placed into the same cage with the experimental mice, and the 2 were allowed to freely interact for 10 min. Animal behavior was captured with a video camera. If excessive tissue damage occurred, the test was prematurely terminated and not analyzed. Mice that exhibited total attack time $>40 \%$ of the test period, or individually housed mice that exhibited total attack time $<3 \%$ of the test period, during the baseline aggression test were eliminated from further analysis (Hong et al., 2014). Videos of behavioral tests were reviewed and hand-scored by a researcher blind to the experimental conditions. Aggressive behaviors, such as chasing, boxing, pinning, and wrestling, were identified as reported (Blanchard and Blanchard, 1977; Lin et al., 2011; Koolhaas et al., 2013; Hong et al., 2014; Golden et al., 2016). Nonaggressive social behavior was defined as any nonaggressive interactions, excluding mating, in which experimental mice were within $1 \mathrm{~cm}$ of the conspecific and displayed characteristic signs of nonaggressive social behavior, such as anogenital sniffing, investigation, and flank rubbing (Mackintosh and Grant, 1963; Olivier, 1981). Self-grooming was identified by paw-licking, facial, and body grooming (Hong et al., 2014).

Sociability test. Sociability test was modified from existing protocols (Silverman et al., 2010). In brief, mice were transferred to the behavioral testing room and allowed to acclimate for at least $1 \mathrm{~h}$. During testing, mice were placed into a $49 \times 49 \mathrm{~cm}$ arena with two inverted wire cups: one empty and the other containing an unfamiliar conspecific. Subject mice were allowed to freely investigate the arena for $30 \mathrm{~min}$. All experiments were conducted under light with a luminescence level of 20 lux at the bottom of the arena (Kaidanovich-Beilin et al., 2011). Social interaction was analyzed using TopScan software (CleverSys) and scored as the ratio of time spent within $5 \mathrm{~cm}$ of the cup containing the animal over the empty cup.

Open field test. Mice were transferred to the behavioral testing room at least $1 \mathrm{~h}$ before testing and then placed into a $49 \times 49 \mathrm{~cm}$ open field arena for $30 \mathrm{~min}$ to freely roam. Distance traveled in the arena was analyzed using TopScan software (CleverSys).

Foot shock, contextual fear memory test, and fear memory extinction. Two-month-old male C57BL/6 mice were individually housed for 3 weeks in a reverse light cycle as described above. Foot shock protocol, which is a commonly used procedure to induce traumatic stress in mice, was adapted from Rau et al. (2005). On day 1, mice were taken to a behavior room and allowed to acclimate for $1 \mathrm{~h}$ before being placed into a fear conditioning chamber illuminated with white light (Context A) within a sound-attenuating cubicle (Med Associates). After a 3 min exploration period, 15 electric shocks $(0.4 \mathrm{~mA}, 1 \mathrm{~s}$ in duration) were administered through an electrified grate at random intervals of 240-480 s over the course of $90 \mathrm{~min}$. Control mice did not receive foot shock. Mice were transferred from their home cage by gently picking the animal up by the base of the tail, placing them into a soup cup, and then placing them inside the fear conditioning chamber. Mice were returned to their home cages using the same method.

For the contextual fear memory test, at $1 \mathrm{~d}$ after foot shock in Context A, mice were placed into the fear conditioning box modified with white plastic walls, no ambient light, and a background odor of $1 \%$ acetic acid (Context B). Mice were left to freely roam within the chamber for $192 \mathrm{~s}$ before a single $1 \mathrm{~s}$, $0.4 \mathrm{~mA}$ shock was delivered via the electrified grate, and were removed from the chamber at $32 \mathrm{~s}$ after foot shock. On the following day, mice were placed into Context B for $8 \mathrm{~min}$ and $32 \mathrm{~s}$ and monitored for freezing behavior. For fear memory extinction, mice were placed into Context $\mathrm{A}$ for $15 \mathrm{~min}$ for 4 consecutive days starting $1 \mathrm{~d}$ after foot shock in Context A. Freezing behavior was defined as an absence of all movement, excluding respiration, and was analyzed with Video Freeze software (Med Associates). 
In vivo optogenetic stimulation

Optogenetic stimulation was performed via an optical fiber (ferrule fiber, ThorLabs) connected through a zirconia split sleeve and patch cord to a $473 \mathrm{~nm}$ laser (Coherent) or a $561 \mathrm{~nm}$ laser (CrystaLaser) under the control of an Optogenetics TTL Pulse Generator (Doric Lenses). Mice expressing ChR2 were stimulated using 1-3 $\mathrm{mW} 473 \mathrm{~nm}$ light at the designated frequencies, pulse durations, and number of trains. Mice expressing ArchT were delivered a 1-3 mW continuous 1 s $561 \mathrm{~nm}$ light pulse.

\section{Electrophysiology}

Slice physiology. Mice were decapitated following anesthesia with isoflurane. The brain was removed and placed in $\mathrm{N}$-methylD-glucamine (NMDG)-based cutting buffer bubbled with 95\% $\mathrm{O}_{2} / 5 \% \mathrm{CO}_{2}$, composed of the following (in $\mathrm{mM}$ ): 93 NMDG, 2.5 $\mathrm{KCl}, 1.2 \mathrm{NaH}_{2} \mathrm{PO}_{4}, 30 \mathrm{NaHCO}_{3}, 20$ HEPES, 25 glucose, 5 sodium ascorbate, 2 thiourea, 3 sodium pyruvate, $10 \mathrm{MgSO}_{4}$, and $0.5 \mathrm{CaCl}_{2}$. After cutting, slices were recovered in NMDG-based buffer for no more than $15 \mathrm{~min}$ at $32^{\circ} \mathrm{C}$ and then transferred to a submerged type recording chamber.

For whole-cell patch-clamp recordings, slices were perfused with ACSF composed of the following (in $\mathrm{mM}$ ): $124 \mathrm{NaCl}, 2.5$ $\mathrm{KCl}, 2 \mathrm{CaCl}_{2}, 2 \mathrm{MgSO}_{4}, 1.2 \mathrm{NaH}_{2} \mathrm{PO}_{4}, 24 \mathrm{NaHCO}_{3}, 5 \mathrm{HEPES}$, and 25 D-glucose and was bubbled with 95\% $\mathrm{O}_{2} / 5 \% \mathrm{CO}_{2}$. All recordings were made at $28^{\circ} \mathrm{C}-30^{\circ} \mathrm{C}$. A glass electrode (6-8 $\mathrm{M} \Omega$ ) was filled with a potassium gluconate-based solution composed of the following (in $\mathrm{mM}$ ): 130 potassium gluconate, $10 \mathrm{Na}$-gluconate, 10 HEPES, 10 phosphocreatine, $4 \mathrm{Mg}$-ATP, $0.3 \mathrm{Na}_{2}$-GTP, and $4 \mathrm{NaCl}$ ). Electrical signals were collected with a MutiClamp 700B Amplifier (Molecular Devices) and digitized at $10 \mathrm{kHz}$ with Digidata 1440 using the Pclamp 10.2 software. After break-ins, only neurons with a resting membrane potential $<-55 \mathrm{mV}$ were used for further analyses. Step currents were injected for induction of action potentials or hyperpolarization. To record lightinduced EPSCs, cells were held at $-70 \mathrm{mv}$ in voltage-clamp mode; 473 or $561 \mathrm{~nm}$ light pulses were generated from a laser (Coherent, Crystalser) and delivered to the slice through an optical fiber (200 $\mu \mathrm{m}$ in diameter). The laser was controlled by Master-8 Pulse Stimulator (A.M.P.I.).

Optrode fabrication. A microdrive was assembled from 3Dprinted pieces, screws, and nuts, and then attached to a nanominiature connector (Omnetics) with epoxy glue. The optrode was constructed with $16 \quad 30$ - $\mu$ m-diameter tungsten wires (California Fine Wire) surrounding a 100- $\mu \mathrm{m}$-diameter optical fiber. One end of the tungsten wire extended $\sim 300 \mu \mathrm{m}$ beyond the tip of the optical fiber, and the other end was wired to pins of a nano-miniature connector. Impedance of each channel in the optrode was measured (usually $\sim 100 \mathrm{k} \Omega$ at $1 \mathrm{kHz}$ ) after construction.

In vivo electrophysiological recording in awake, freely moving mice. The nano-miniature connector on the microdrive was plugged into an amplifier (RHD2132, Intan Technologies). The amplifier was connected to an RHD2000 USB interface board (Intan Technologies) through a motorized commutator (Tucker Davis Technology). Electrical signals were filtered to obtain signals between 1 and $7500 \mathrm{~Hz}$, sampled and digitized at $30 \mathrm{kHz}$ by the amplifier, and recorded by RHD2000 Interface software (Intan Technologies). The $473 \mathrm{~nm}$ laser (Coherent) was controlled by a USB-6212 Bus-Powered DAQ Device (National Instruments) in Labview (National Instruments) virtual instruments. After a 2 week recovery period, mice implanted with optrodes were placed into high-walled novel cages inside a grounded Faraday cage and allowed to acclimate for $10 \mathrm{~min}$ before recording. Video recordings of animal behaviors were obtained via a ceiling-mounted acA1300-200uc USB 3.0 camera (Basler) at 30 frames per second simultaneously with electrophysiological recording. Video and electrophysiological recordings were synchronized using Master-8 Pulse Stimulator (A.M.P.I.), which generated and sent an electrical signal for each light pulse and video frame to the RHD2000 recording software (Intan Technologies). Local field potentials (LFPs) were evoked by delivering 1-3 mW, $1 \mathrm{~ms}$ light pulses at $0.05 \mathrm{~Hz}$ through the implanted optical fiber. Laser power was adjusted to elicit fEPSPs with a clear early and late component. Optically evoked fEPSPs were analyzed by obtaining the slope derived by fitting the rising phase of the late component of the fEPSP (excluding the bottom and top 10\%) with linear regression as described previously (Xiong et al., 2015; Zhou et al., 2017). In vivo electrophysiological data were analyzed with custom-written MATLAB scripts. Only mice with baseline fEPSPs not significantly different from each other as determined by repeated-measures ANOVA test, verified opsin expression, and correct targeting of optrodes were included in further analysis.

Production of GFP virus. HEK-293T cells were cultured on 15 $\mathrm{cm}$ plates coated with $0.1 \%$ gelatin in DMEM supplemented with 10\% FBS (Thermo Fisher Scientific). When the cell reached $90 \%$ confluence, the medium was changed at $2 \mathrm{~h}$ before transfection. For transfection of each $15 \mathrm{~cm}$ plate, $10 \mu \mathrm{g}$ pRRlsin.CMV:eGFP, $7.5 \mu \mathrm{g}$ psPAX2, $2.5 \mu \mathrm{g}$ pMD2G, and $1 \mu \mathrm{g}$ pAdVantage packaging vector were added to $2 \mathrm{ml}$ water containing $260 \mu \mathrm{l} \mathrm{CaCl}_{2}(2 \mathrm{M})$, and then mixed with $2 \mathrm{ml} 2 \times$ HBSS (50 mM HEPES, $280 \mathrm{~mm}$ $\mathrm{NaCl}, 1.5 \mathrm{~mm} \mathrm{Na}_{2} \mathrm{HPO}_{4}, \mathrm{pH}$ 7.05). After incubation at room temperature for $2 \mathrm{~min}$, the mixture was added to the culture plate dropwise. The medium was replaced with $15 \mathrm{ml}$ UltraCULTURE medium (Lonza Bioscience, $1 \mathrm{~mm}$ sodium pyruvate, $0.075 \%$ sodium bicarbonate, $1 \times$ glutamine) at $16 \mathrm{~h}$ after transfection. The medium was removed $48 \mathrm{~h}$ after transfection and kept at $4^{\circ} \mathrm{C} ; 15$ $\mathrm{ml}$ fresh UltraCULTURE medium was added to the plate and collected $72 \mathrm{~h}$ after transfection. The media collected at the two times were combined, filtered with $0.45 \mu \mathrm{m}$ filter bottles, and centrifuged at 25,000 rpm for $90 \mathrm{~min}$ at $4^{\circ} \mathrm{C}$ (Beckman, SW28 rotor). The supernatant was removed, and the pellet containing the virus was dissolved by incubation with $100 \mu \mathrm{l} 1 \times$ HBSS overnight at $4^{\circ} \mathrm{C}$. For further purification of virus, the viral suspension was placed on the top of $1.5 \mathrm{ml} 20 \%$ sucrose (in $1 \times$ HBSS) and centrifuged at 21,000 rpm for $2 \mathrm{~h}$ at $4^{\circ} \mathrm{C}$ (Beckman, SW55 rotor). The pellet was incubated with $100 \mu \mathrm{l} 1 \times$ HBSS overnight at $4^{\circ} \mathrm{C}$, aliquoted, and stored at $-80^{\circ} \mathrm{C}$. The titer of purified virus was determined by transducing HEK-293T cells with a series of dilutions. All viruses used for in vivo injection had a titer of $10^{9}$ $10^{10} \mathrm{IU} / \mathrm{ml}$.

\section{Immunohistochemistry}

Mice were transcardially perfused with $4 \%$ PFA in PBS solution. Brains were removed and postfixed at $4^{\circ} \mathrm{C}$ overnight, then cryoprotected overnight in $15 \%$ sucrose (in PBS) followed by $30 \%$ sucrose in PBS. Brains were cut into $30-\mu \mathrm{m}$-thick sections using a cryostat (CM3050 S, Leica Microsystems), then either mounted onto silanized slides (KD Medical) or stored in PBS as floating sections for immunohistochemistry or confirming the location of viral injection and implantation. For immunohistochemistry, free-floating brain sections were heated to $80^{\circ} \mathrm{C}$ for $30 \mathrm{~min}$ in citrate buffer for antigen retrieval (Jiao et al., 1999) and then blocked with $10 \%$ goat serum and 1\% BSA in PBS-T (PBS with $0.03 \%$ Triton $\mathrm{X}-100$ ) for $3 \mathrm{~h}$ at room temperature. Sections were 
then stained for primary antibodies overnight at $4^{\circ} \mathrm{C}$, followed by incubation with secondary antibodies for $1 \mathrm{~h}$ at room temperature. Sections were mounted to slides with Vectashield HardSet Antifade Mounting Medium containing DAPI (Vector Laboratories).

\section{Image acquisition and image analysis}

Brain slices were imaged with a multislide fluorescent microscope (Axio Scan, Carl Zeiss) with a $10 \times$ (NA 0.45) objective to locate the areas with fluorescence signals, and then a laser scanning confocal microscope (LSM510 and LSM700, Carl Zeiss) with a $40 \times$ (NA 1.3 oil immersion) objective for high-magnification imaging in the ROI. The location of optrodes was determined from the electrode tract left in the brain tissue. In some images, boundaries can be seen between two adjacent areas due to uneven illumination between tiles. $z$-stack confocal images were collapsed and analyzed with ImageJ by researcher blind to the experimental conditions. c-Fos-positive cells were identified using the Analyze Particles function of Image $\mathrm{J}$ and validated as cells by their overlap with DAPI. Cells colabeled for c-Fos, DAPI, and $\mathrm{CaMKII} \alpha$, calbindin, calretinin, or somatostatin were manually counted by a researcher blind to the experimental conditions.

\section{Statistical analysis}

All data were presented as individual data points or expressed as mean \pm SEM. SigmaPlot software was used for statistical analysis. To compare aggression Test 1 to aggression Test 2 in priming experiments, paired Student's $t$ test was used. To compare two groups, Student's $t$ test was used if the data were normally distributed with equal variance, and Mann-Whitney $U$ test was used if the data did not satisfy both the normality and equivariance tests. To compare three or more groups, one-way ANOVA was used and Holm-Sidak test was used for post hoc multiple comparisons to identify groups that were significantly different. To analyze three or more groups injected with two different viruses, two-way ANOVA was used and Holm-Sidak test was used for post hoc multiple comparisons to identify groups that were significantly different. To analyze in vivo electrophysiological data, repeated-measures ANOVA was used. $p<0.05$ was considered significant, and all tests were two-tailed.

\section{Results \\ Aggression priming activates CaMKII $\alpha^{+}$neurons in the MeApv}

To interrogate the mechanism of experience-dependent modification of aggressive behavior, we focused on "aggression priming," which is the enhancement of aggressive behavior by an agnostic encounter (Largerspetz, 1969; Tellegen and Horn, 1972; Potegal and Popken, 1985; Potegal, 1992). Aggression was tested in a neutral arena which facilitates the first attack and allows for the full expression of aggressive behaviors (Olivier and van Dalen, 1982; Roubertoux et al., 1999; Miczek et al., 2001). The test was started by placing a male subject mouse (C57BL/6, 8week old, individually housed for 3 weeks) into a test cage for $30 \mathrm{~min}$ before introduction of an unfamiliar, smaller male conspecific (Fig. 1A). Mice were allowed to freely interact for $10 \mathrm{~min}$, and then the conspecific was removed (Fig. 1A). Most subject mice we tested ( 15 of 22 ) attacked the conspecific during this period and are hereon referred to as aggressors (AGG), whereas mice that did not attack are referred as NON. A new conspecific was placed into the test cage $30 \mathrm{~min}$ after the first interaction session for the second aggression test (Fig. 1A).
The AGG mice exhibited distinct offensive aggressive behaviors as reported in previous studies, such as rear and lateral attacks, boxing, chasing, and wrestling (which includes pin downs and clinch attacks) (Blanchard and Blanchard, 1977, 2003a,b; Miczek and O’Donnell, 1978; Potegal et al., 1996b; de Boer et al., 2003) (Fig. 1B). During the second aggression test, AGG mice spent more time attacking and initiated the first attack with a shorter latency, indicating that aggression priming by the first session was successful (Fig. $1 C-F$ ). The nonaggressive social behaviors of AGG and NON mice were comparable during the first and the second interaction period (Fig. 1G-J).

Next, we assessed whether MeApv neurons are activated by aggression priming through analysis of c-Fos expression. To this end, the brains of the subject mice were removed $1 \mathrm{~h}$ after aggression priming for immunostaining with a c-Fos antibody along with antibodies against CaMKII $\alpha$ or markers of GABAergic neurons present in the MeA, including calbindin, calretinin, somatostatin, and paravalbumin (PV) (Butler et al., 2011; Keshavarzi et al., 2014). We chose CaMKII $\alpha$ because while it is known to be expressed by non-GABAergic neurons in many brain regions including the MeA (Benson et al., 1992; Jones et al., 1994; Liu and Jones, 1996; Wallen-Mackenzie et al., 2009; Butler et al., 2011; X. J. Wang et al., 2013), the functional role of CaMKII $\alpha$ neurons in aggression has not been explored.

While cell density of the MeApv was comparable in AGG and NON mice, more c-Fos ${ }^{+}$cells were detected in AGG mice than in NON mice (Fig. 2A-D). This is consistent with earlier reports that MeApv cells are activated by conspecific intermale aggression (Kollack-Walker and Newman, 1995; Lin et al., 2011). Within the MeApv of AGG mice, $64.3 \pm 6.3 \%$ of $^{c}-F_{0 s}{ }^{+}$cells expressed CaMKII $\alpha$ with fewer cells expressing GABAergic cell markers $(9.7 \pm 4.4 \%$ for calbindin, $4.9 \pm 1.3 \%$ for calretinin, $3.0 \pm 0.8 \%$ for somatostatin, $0 \pm 0 \%$ for PV; Fig. 2E-J). Moreover, CaMKII $\alpha$-expressing neurons of the MeApv had the greatest proportion of ${\mathrm{c}-\mathrm{Fos}^{+}}^{+}$cells $(35.1 \pm 8.9 \%$ for CaMKII $\alpha$, $12.3 \pm 6.8 \%$ for calbindin, $14.1 \pm 3.0 \%$ for calretinin, $3.3 \pm 1.3 \%$ for somatostatin, and $0 \pm 0 \%$ for PV; Fig. $2 K)$.

It has been reported that CaMKII $\alpha$ is primarily expressed by excitatory neurons in many brain regions (Benson et al., 1992; X. J. Wang et al., 2013). To test whether it applies to MeApv, we injected Cre-dependent adeno-associated virus (AAV) GFP into the MeApv of mice expressing Cre recombinase driven by the vesicular glutamate transporter Type 2 ( $v$ Glut2) promoter. $v$ Glut 2 is widely expressed by glutamatergic neurons throughout the MeApv (Choi et al., 2005; Cheong et al., 2015; Ishii et al., 2017). At 3 weeks after surgery, $>85 \%$ of $\mathrm{GFP}^{+}$cells were positive for CaMKII $\alpha$ (Fig. $2 L, M$ ). Hence, the majority of CaMKII $\alpha$ cells in the MeApv are excitatory.

Together, these results indicate that CaMKII $\alpha$ cells of the $\mathrm{MeApv}$ are activated by aggression priming.

\section{Low-frequency photostimulation (LFPS) of the MeApv abolishes aggression priming}

We next examined the role of MeApv CaMKII $\alpha$ neurons in aggression priming. It has been reported that high-frequency electrical stimulation at the MeA mimics the effect of aggression priming on aggressiveness (Potegal et al., 1996a). High-frequency stimulation can induce NMDAR-dependent synaptic potentiation in various brain regions, including the MeA (Shindou et al., 1993; Watanabe et al., 1995, 1996; Smerin et al., 2016). Therefore, it is possible that synaptic potentiation is involved in aggression priming. To test this possibility, we exposed mice to 


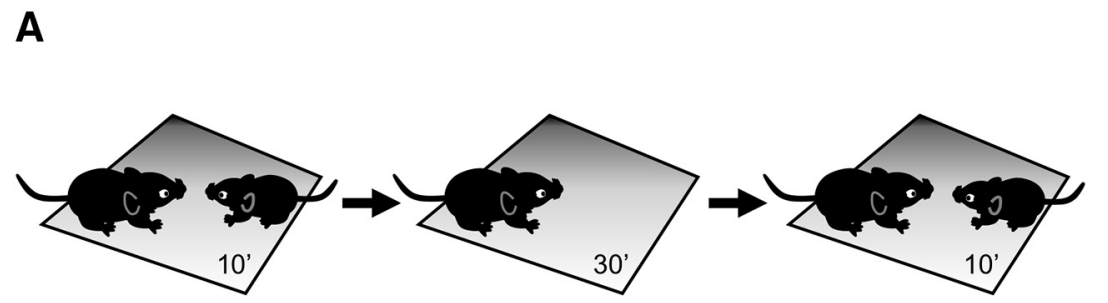

Test 1 (Aggression Priming)
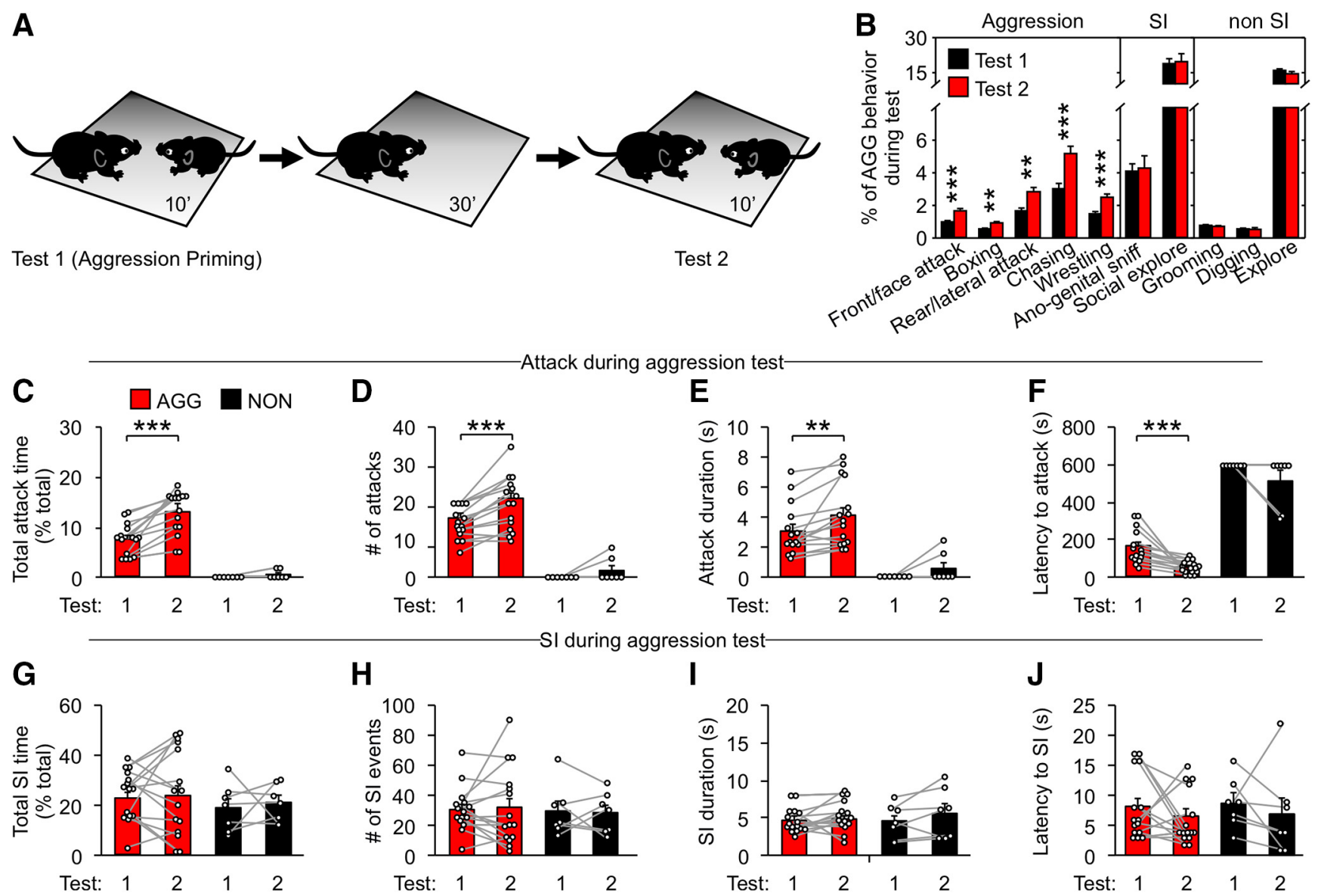

Figure 1. Characterization of aggression priming in mice. $\boldsymbol{A}$, Experimental schedule for aggression priming testing. Each mouse was used for only one round of experiment. $\boldsymbol{B}$, Percentage of stereotyped behaviors performed during first and second aggression test (SI: social interaction). Two-tailed paired Student's $t$ test was used for statistical analysis ( $n=15$ mice): front/face attack, $t_{(14)}=-4.004, p<0.001$; boxing, $t_{(14)}=-3.433, p=0.004$; rear/lateral attack, $t_{(14)}=-3.167, p=0.007$; chasing, $t_{(14)}=-4.242, p<0.001 ;$ wrestling, $t_{(14)}=-6.19, p<0.001$; anogenital sniff, $t_{(14)}=-0.261, p=0.798$; social investigation, $t_{(14)}=-0.236, p=0.817$; grooming, $t_{(14)}=1.703, p=0.111$; digging, $t_{(14)}=1.171, p=0.261$; explore, $t_{(14)}=1.144$, $p=0.272$. $(-J$, Aggressive $(\boldsymbol{C}-\boldsymbol{F})$ and nonaggressive social interaction $(\boldsymbol{G}-\boldsymbol{J})$ behavior in aggressive (AGG, $n=15)$ and nonaggressive (NON, $n=7$ ) mice during the priming experiment. Twotailed paired Student's $t$ test was used for statistical analysis. AGG attack: $\boldsymbol{C}, t_{(14)}=-6.190, p<0.001 ; \boldsymbol{D}, t_{(14)}=-6.190, p<0.001 ; \boldsymbol{E}, t_{(14)}=-3.220, p=0.006, \boldsymbol{F}, t_{(14)}=4.254$, $p<0.001$. NON attack: $\boldsymbol{C}, t_{(6)}=-1.549, p=0.172 ; \boldsymbol{D}, t_{(6)}=-1.549, p=0.172 ; \boldsymbol{E}, t_{(6)}=-1.500, p=0.184 ; \boldsymbol{F}, t_{(6)}=1.548, p=0.173$. AGG SI: $\boldsymbol{G}, t_{(14)}=-0.256, p=0.802 ; \boldsymbol{H}, t_{(14)}=$ $-0.302, p=0.767 ; \boldsymbol{I}, t_{(14)}=-0.322, p=0.752 ; J, t_{(14)}=0.955, p=0.356$. NON SI: $\boldsymbol{G}, t_{(6)}=-0.318, p=0.761 ; \boldsymbol{H}, t_{(6)}=0.197 ; p=0.850 ; \boldsymbol{I}, t_{(6)}=-1.470, p=0.192 ; J, t_{(6)}=0.660$, $p=0.534$. Data are mean \pm SEM. ${ }^{* *} p<0.01,{ }^{* * *} p<0.001$.

an optical LTD protocol (Nabavi et al., 2014; Bagot et al., 2015; Zhu et al., 2016; Kim and Cho, 2017; Zhou et al., 2017) after aggression priming. To this end, 7- to 8-week-old male mice were bilaterally injected with AAV expressing the superfast channelrhodopsin 2 variant E123A (hereon referred to as ChR2) fused with GFP or GFP alone under control of the CaMKII $\alpha$ promoter into the MeApv. At 3 weeks after surgery, ChR2 expression indicated by GFP fluorescence was readily detectable in the MeApv (Fig. 3A-E).

To examine the cell types infected by AAV, we stained brain sections of injected mice with the antibodies described above. $\mathrm{GFP}^{+}$cells did not express the GABAergic neuron markers that we tested, and the majority of $\mathrm{GFP}^{+}$cells were positive for CaMKII $\alpha$ (Fig. 3A-F). This finding is consistent with the previous report that $\mathrm{AAV}$ with the $\mathrm{CaMKII} \alpha$ promoter preferentially labels excitatory neurons (Nathanson et al., 2009).

To test whether optogenetic stimulation of ChR2 expressed in the MeApv effectively activates neurons, we recorded MeApv neurons in brain slices of mice injected with ChR2 virus using whole-cell patch-clamp. Neurons were stimulated with $473 \mathrm{~nm}$ light pulses $\left(2 \mathrm{~ms}\right.$ pulse duration, $\left.15 \mathrm{mw} / \mathrm{mm}^{2}\right)$ at various frequencies $(5-100 \mathrm{~Hz})$. Whole-cell recordings of MeApv neurons in voltage-clamp mode detected light-evoked depolarizing currents in all recorded cells at all tested frequencies (Fig. $3 G, H$ ). Hence, optogenetic stimulation of MeApv neurons induces neural depolarization.

We applied the optical LTD protocol (900 pulses of $473 \mathrm{~nm}$ light at $1 \mathrm{~Hz}$; LFPS) to the MeApv of the subject mouse immediately after aggression priming and introduced an unfamiliar conspecific $30 \mathrm{~min}$ after the onset of light stimulation (Fig. 3I). While aggression priming increased aggressive behaviors in GFP-injected mice regardless of LFPS, ChR2-injected mice stimulated with LFPS became less aggressive during the second aggression test (Fig. 3J-M). Nonaggressive social behaviors were unchanged by LFPS (Fig. $3 N-Q$ ). These results indicate that LFPS of MeApv neurons suppresses the priming effect of attack on aggression.

\section{HFPS of the MeApv mimics aggression priming}

The LFPS effect suggests that synaptic potentiation in the MeApv circuits may underlie aggression priming. To test this possibility, we first applied $100 \mathrm{~Hz}$ photostimulation, which induces LTP in vivo and alters behavior (Nabavi et al., 2014; 

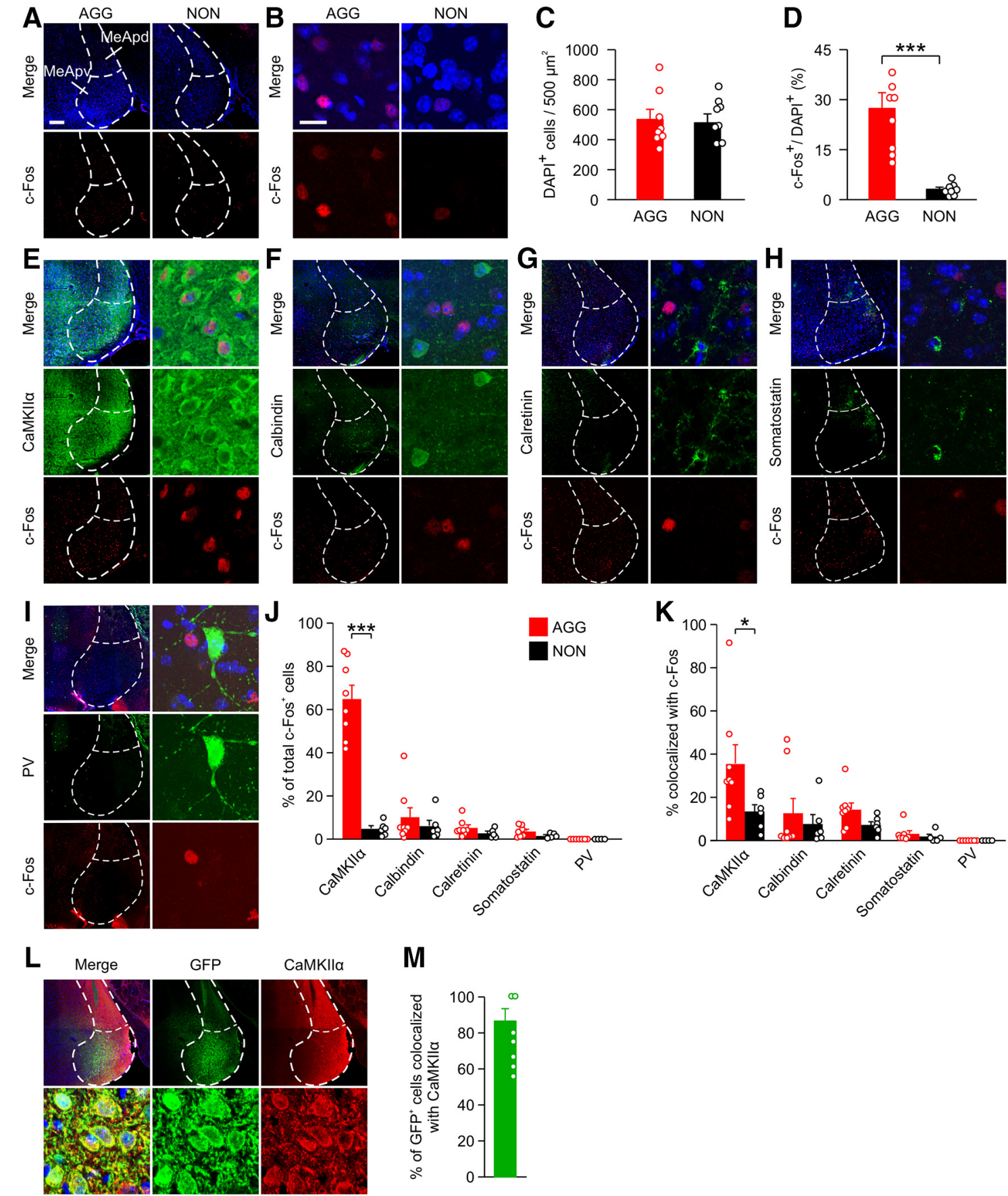

\section{M}

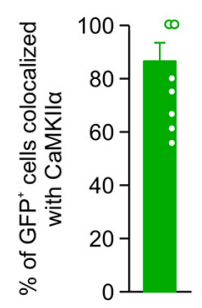

Figure 2. CaMKIII $\alpha^{+}$neurons of the MeApv are activated by aggression priming. Mice ( 9 weeks of age) were placed in the test cage and either allowed to freely interact with a submissive conspecific male for $10 \mathrm{~min}$ or left alone in the cage for the same amount of time. The mice were perfused $60 \mathrm{~min}$ after the interaction period for immunostaining. In some images, boundaries can be seen between two adjacent areas due to uneven illumination between tiles. $\boldsymbol{A}, \boldsymbol{B}$, Representative images of brain sections of $\mathrm{c}-\mathrm{Fos}^{+}$cells in the MeApv from aggressive (AGG, $n=8$ ) or nonattacking control (NON, $n=8$ ) animals after aggression priming. $\boldsymbol{C}$, Quantification of total number of DAPI ${ }^{+}$cells for $\boldsymbol{A}$ and $\boldsymbol{B}$. Two-tailed paired Student's $t$ test was used for statistical analysis: $t_{(14)}=0.0706, p=0.945$. D, Quantification of $c$-Fos ${ }^{+}$cells for $\boldsymbol{A}$ and $\boldsymbol{B}$. Mann-Whitney test was used for statistical analysis of Test 1 to Test $2: U=0.000, p<0.001$. $\boldsymbol{E}-\boldsymbol{I}, \mathbf{R e p r e s e n t a t i v e}$ images of brain sections from AGG mice costained for c-Fos and DAPI along with CaMKIll $\alpha(\boldsymbol{E})$, calretinin $(\boldsymbol{F})$, calbindin $(\boldsymbol{G})$, somatostatin $(\boldsymbol{H})$, or PV $(\boldsymbol{I})$. $\boldsymbol{J}, \boldsymbol{K}$, Quantification of $c-$ Fos ${ }^{+}$cells for each cell type from AGG $(n=8)$ or NON mice $(n=6)$ after aggression priming. \% of c-Fos ${ }^{+}$cells that colocalize with the indicated antibodies are shown in $\boldsymbol{J}$. Mann-Whitney test was used for statistical analysis of versus NON: CaMKII $\alpha, U=0.000, p<0.001$; calretinin, $U=20.000, p=0.662$; calbindin, $t_{(11)}=1.291, p=0.223$; somatostatin, $t_{(10)}=1.453, p=0.177 ;$ PV, $U=14.000$, $p=1.000$. \% of cells positive for each antibody that colocalize with c-Fos are shown in $\boldsymbol{K}$. Mann-Whitney test was used for statistical analysis of AGG versus NON: CaMKII $\alpha, t_{(12)}=2.537$, $p=0.0261$; calretinin, $t_{(12)}=-0.944, p=0.364$; calbindin, $t_{(12)}=-1.968, p=0.0727$; somatostatin, $U=12.000, p=0.432 ; \mathrm{PV}, U=14.000, p=1.000 . L, M, v G /$ t $2^{+}$cre-line mice $(7$ weeks of age) were injected with cre-dependent GFP AAV into the MeApv and then used for immunolabeling 3 weeks later. $L$, Representative images of brain sections from GFP-injected animals stained for CaMKII $\alpha$. In some images, boundaries can be seen between two adjacent areas due to uneven illumination between tiles. $\boldsymbol{M}$, Quantification of $\boldsymbol{L}$ showing $\%$ of GFP ${ }^{+}$cells that are positive for CaMKIl $\alpha$. Only cells within the MeApv were counted. Scale bars: $A, 2 \mathrm{~mm} ; \boldsymbol{B}, 100 \mu \mathrm{m}$. Data are mean \pm SEM. ${ }^{*} p<0.05$, ${ }^{* * *} p<0.001$. 

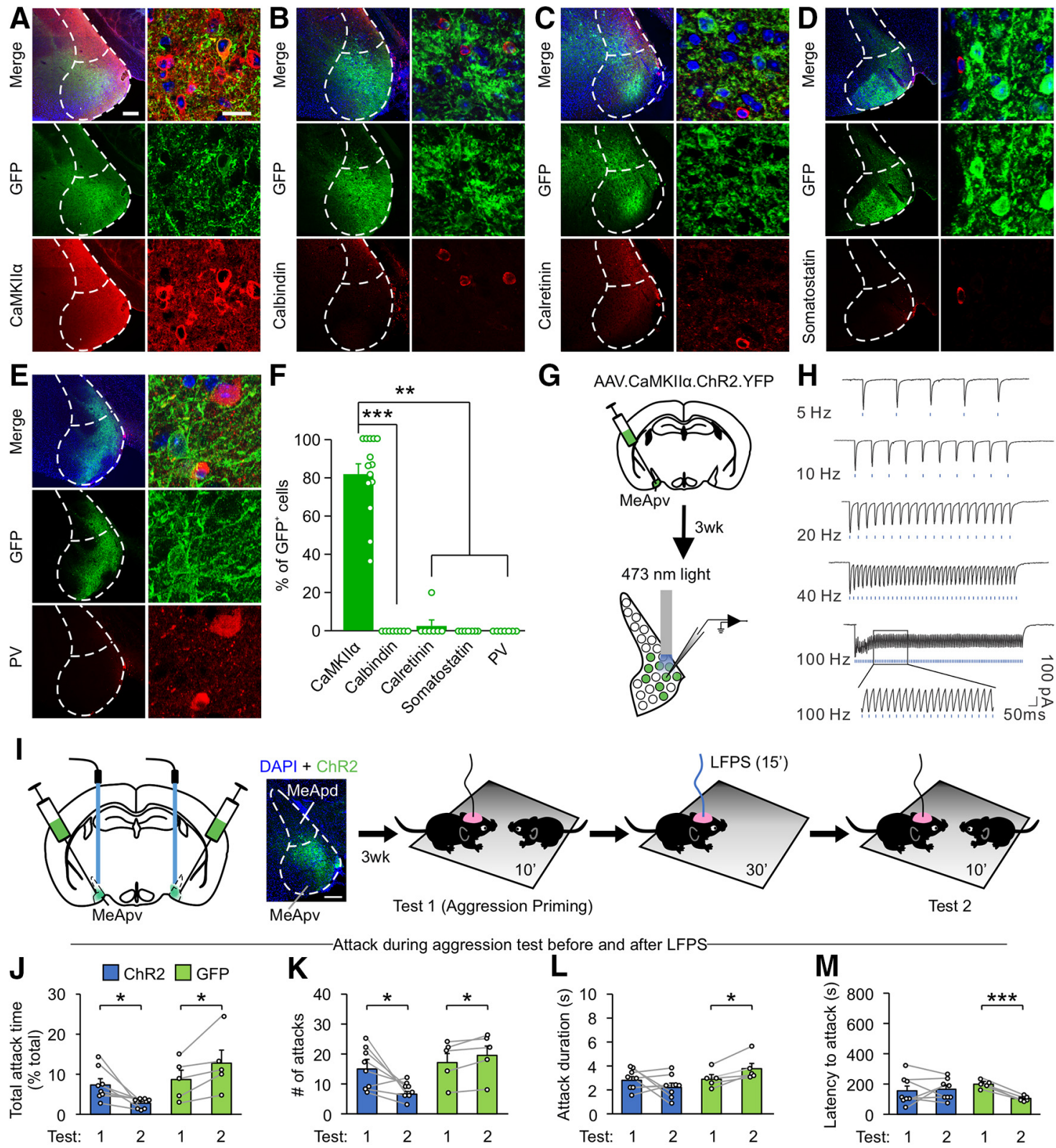

K
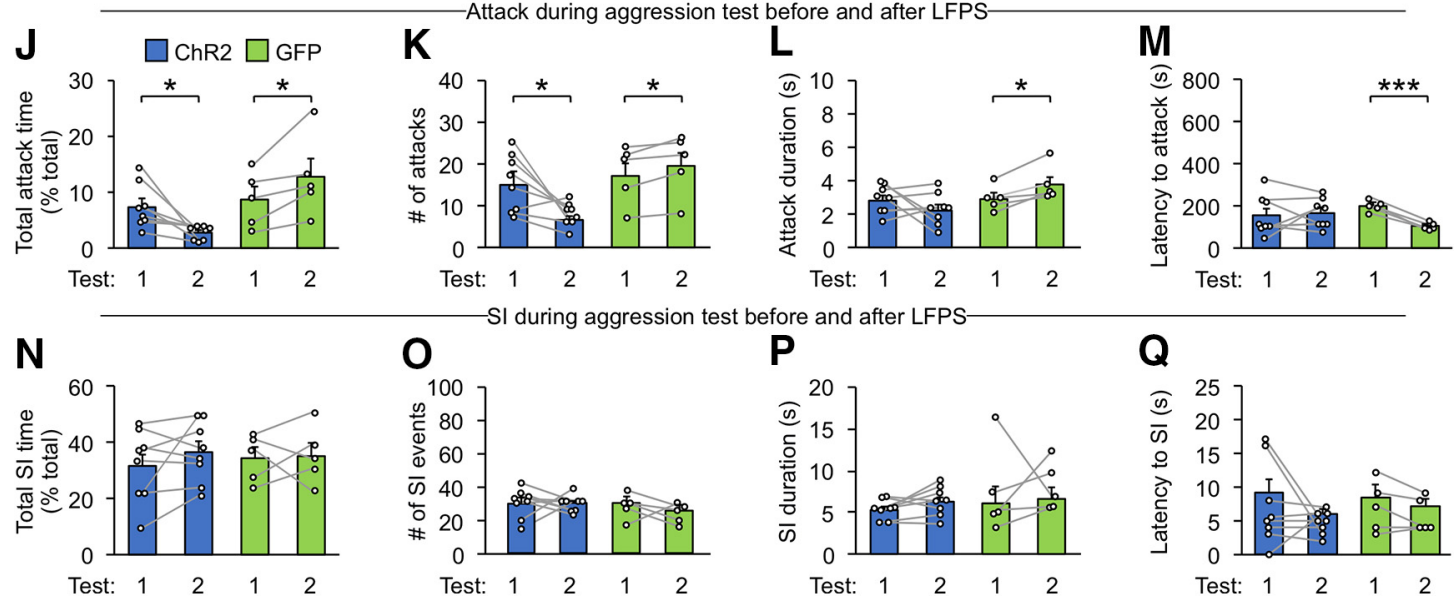

Figure 3. LFPS of CaMKII $\alpha^{+}$neurons of the MeApv suppresses aggression priming. Mice (7 weeks of age) were bilaterally injected with GFP or ChR2 AAV under control of the CaMKIll $\alpha$ promoter into the MeApv and then used for immunolabeling $(\boldsymbol{A}-\boldsymbol{F})$, slice electrophysiology $(\mathbf{G}, \boldsymbol{H})$, or aggression testing $(\boldsymbol{I}-\mathbf{Q}) \mathbf{3}$ weeks later. $\boldsymbol{A}-\boldsymbol{E}$, Representative images of brain sections from GFP-injected animals stained for CaMKII $\alpha(\boldsymbol{A}, n=14$ mice), calretinin ( $\boldsymbol{B}, n=8$ mice), calbindin ( $\boldsymbol{C}, n=7$ mice), somatostatin $(\boldsymbol{D}, n=7$ mice), or PV (E, $n=7$ mice). In some images, boundaries can be seen between two adjacent areas due to uneven illumination between tiles. Scale bars: $\boldsymbol{A}$, low-magnification images (left), $2 \mathrm{~mm}$; high-magnification images (right), $100 \mu \mathrm{m}$. $\boldsymbol{F}$, Quantification of $\boldsymbol{A}-\boldsymbol{E}$ showing $\%$ of GFP ${ }^{+}$cells that are positive for the indicated antibodies. Only cells within the MeApv were counted. Kruskal-Wallis one-way ANOVA on Ranks was used for statistical analysis of antibodies colocalized with GFP: $H 4=38.509, p<0.001$. Dunn's method was used for pairwise multiple comparisons: CaMKIll $\alpha$ versus calretinin, $p<0.001 ;$ CaMKIll $\alpha$ versus calbindin, $p=0.006$; CaMKII $\alpha$ versus somatostatin, $p=0.002$; CaMKIl $\alpha$ versus PV, $p=0.006$. All other comparisons are not statistically significant. $\boldsymbol{G}, \boldsymbol{H}$, Experimental schedule for electrophysiological recordings of optically evoked postsynaptic currents in slices containing the MeApv. MeApv neurons were stimulated with light ( $2 \mathrm{~ms}$ pulse duration, $\left.473 \mathrm{~nm}, 15 \mathrm{mw} / \mathrm{mm}^{2}\right)$ to induce EPSCS $(\boldsymbol{H})$. I, Experimental schedule for viral injection, optical fiber placement, and LFPS administration before second aggression test. Each mouse was stimulated only once. In the left diagram, the green area in the MeApv represents the viral spread. Blue stick represents the location of optical fibers. Representative image of ChR2 expression in the MeApv. Scale bar, $200 \mu \mathrm{m}$. J-Q , The effect of LFPS on primed aggressive behavior $(\boldsymbol{J}-\mathbf{M})$ and nonaggressive social interaction $(\boldsymbol{N}-\mathbf{Q})$. Two-tailed paired Student's $t$ test was used to compare aggression Test 1 to Test 2 for AGG $(n=8)$ and GFP $(n=5)$ mice. AGG attack in ChR2 mice: $\boldsymbol{J}, t_{(7)}=2.925, p=0.022 ; \boldsymbol{K}, t_{(7)}=2.934,0.022 ; \boldsymbol{L}, t_{(7)}=1.310, p=0.232 ; M, t_{(7)}=-0.365, p=0.726$. AGG attack in GFP mice: $\boldsymbol{J}, \boldsymbol{L}, t_{(4)}=-2.784, p=0.050 ; \boldsymbol{M}, t_{(4)}=-3.539, p=0.024 ; \boldsymbol{N}, t_{(4)}=-3.644, p=0.022 ; \boldsymbol{O}, t_{(4)}=10.871, p<0.001$. SI in ChR2 mice: $\boldsymbol{J}, t_{(7)}=-1.283, p=0.240 ; \boldsymbol{K}, t_{(7)}=0.089$, 

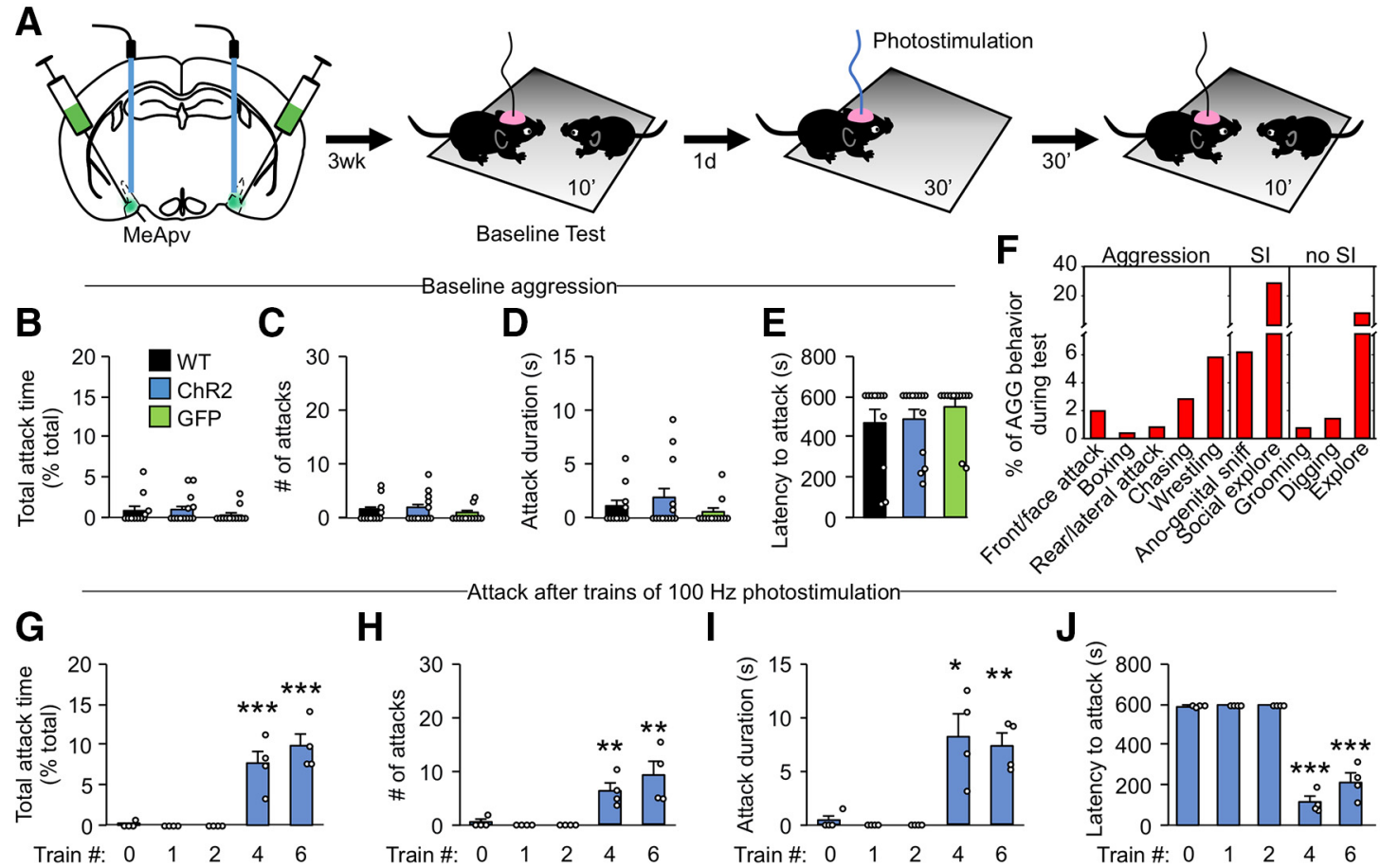

H
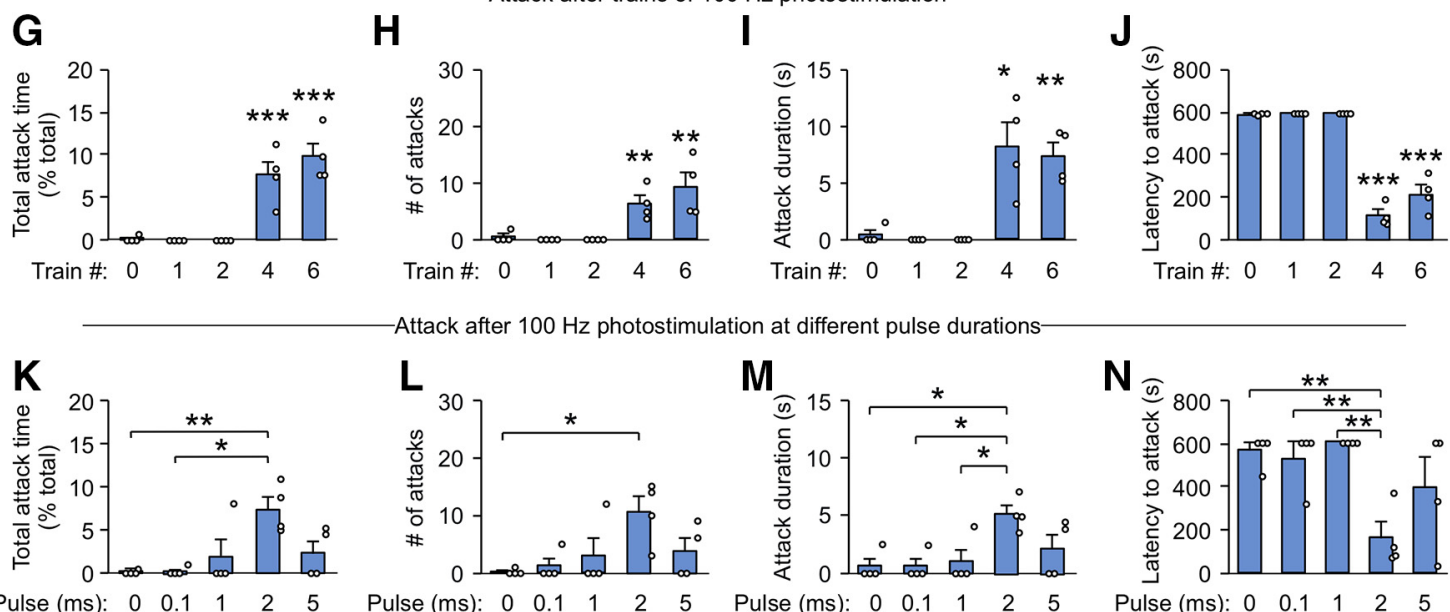

Attack after $100 \mathrm{~Hz}$ photostimulation at different pulse durations-
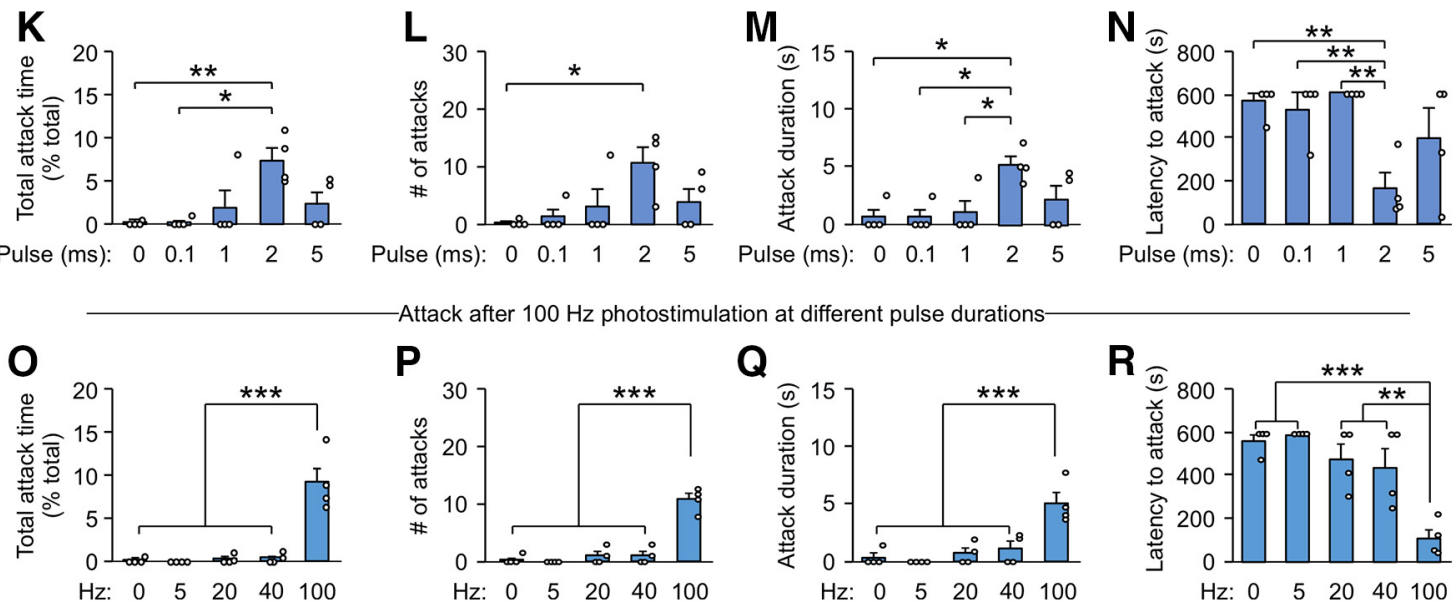

Figure 4. HFPS of MeApv neurons causes an enhancement of aggressive behavior. $A$, Experimental schedule for optical stimulation and aggression testing. Left, Green area in the MeApv represents the viral spread. Blue stick represents the location of optical fibers. $\boldsymbol{B}-\boldsymbol{E}$, Baseline aggression of noninjected and virus-injected mice. One-way ANOVA was used for statistical analysis across groups: $n=11$ WT, 13 ChR2, and 11 GFP mice; $\boldsymbol{B}, F_{(2,33)}=0.656, p=0.526 ; \boldsymbol{C}, F_{(2,33)}=0.561, p=0.576 ; \boldsymbol{D}, F_{(2,33)}=1.077, p=0.352 ; \boldsymbol{E}, F_{(2,33)}=0.639, p=0.534$. $\boldsymbol{F}$, Percentage of stereotyped behaviors performed during aggression test $30 \mathrm{~min}$ after photostimulation of the MeApv using 4 trains (50 pulses per train) of $2 \mathrm{~ms}$ light pulses at $100 \mathrm{~Hz}$ (HFPS). $\mathbf{G}-\boldsymbol{R}$, The effect of $100 \mathrm{~Hz}$ stimulation with different train numbers $(\boldsymbol{G}-\boldsymbol{J}, n=4$ mice for each group), pulse durations $(\boldsymbol{K}-\boldsymbol{N}, n=4$ mice for each group), and frequencies $(\mathbf{0}-\boldsymbol{R}, n=4$ mice for each group) on aggressive behavior. One-way ANOVA was used for statistical analysis across groups: $F_{(4,15)}=22.73$ for $\boldsymbol{G}, 10.028$ for $\boldsymbol{H}, 8.569$ for $\boldsymbol{I}, 116.496$ for $\boldsymbol{J}, 5.386$ for $\boldsymbol{K}, 3.496$ for $\boldsymbol{L}, 4.826$ for $\boldsymbol{M}, 7.178$ for $\boldsymbol{N}, 25.272$ for $\mathbf{0}, 44.897$ for $\boldsymbol{P}, 13.294$ for $\boldsymbol{Q}, 12.212$ for $\boldsymbol{R} ; p<0.001$ for $\boldsymbol{G}, p<0.001$ for $\boldsymbol{H}, p<0.001$ for $\boldsymbol{I}, p<0.001$ for $\boldsymbol{J}, p=0.007$ for $\boldsymbol{K}, p=0.033$ for $\boldsymbol{L}, p=0.011$ for $\boldsymbol{M}, p=0.002$ for $\boldsymbol{N}, p<0.001$ for $\mathbf{0}, p<0.001$ for $\boldsymbol{P}, p<0.001$ for $\boldsymbol{Q}, p<0.001$ for $\boldsymbol{R}$. Holm-Sidak correction was used for multiple comparisons between trains. Data are mean \pm SEM. ${ }^{*} p<0.05$, ${ }^{* *} p<0.01,{ }^{* *} p<0.001$.

Zhou et al., 2017), to mice injected with the ChR2 virus at the MeApv. Mice were prescreened for low baseline aggression levels $1 \mathrm{~d}$ before photostimulation. Baseline aggression was comparable in uninjected mice and mice injected with ChR2 or GFP virus (Fig. $4 A-E$ ). Four trains of $100 \mathrm{~Hz}$ light pulses (50 pulses per train, $2 \mathrm{~ms}$ pulse duration) were delivered to the injected mice,

$0.932 ; \boldsymbol{L}, t_{(7)}=-1.724, p=0.128$. SI in GFP: $J, t_{(4)}=-0.152, p=0.886 ; \boldsymbol{K}, t_{(4)}=0.617$ $p=0.571 ; L, t_{(4)}=-0.620, p=0.569 ; M, t_{(4)}=1.500, p=0.208$. Data are mean \pm SEM. ${ }^{*} p<0.05$. ${ }^{* *} p<0.01$. ${ }^{* * *} p<0.001$. and a smaller, unfamiliar conspecific male mouse was introduced into the test cage $30 \mathrm{~min}$ later (Fig. $4 \mathrm{~A}$ ). Mice stimulated with $100 \mathrm{~Hz}$ stimulation were more aggressive than sham-stimulated mice, while the two groups' baseline aggression tested $1 \mathrm{~d}$ before photostimulation was comparable (Fig. $4 B-E$ ); $100 \mathrm{~Hz}$ stimulation was most effective at $\geq 4$ trains ( 50 pulses per train) with a pulse duration of $2 \mathrm{~ms}$ (HFPS) (Fig. $4 G-N$ ). In contrast to 100 $\mathrm{Hz}$ stimulation, 5, 20, and $40 \mathrm{~Hz}$ stimulations with the same total number of pulses had no effect on aggression (Fig. 4O-R). HFPS induced offensive aggressive behaviors as aggression priming (Fig. 4F). 

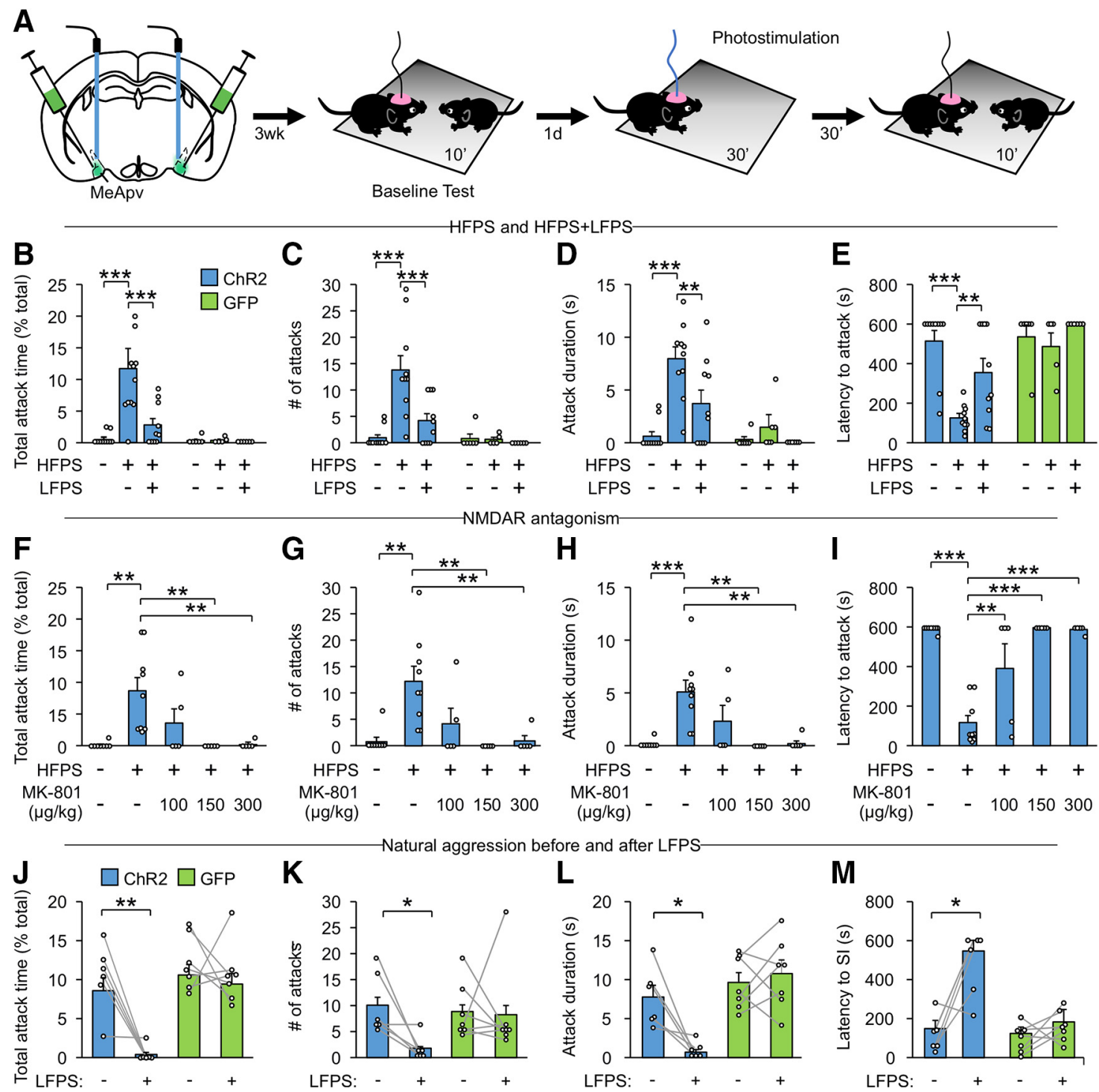

Figure 5. HFPS of MeApv neurons increases aggression through an NMDAR-dependent mechanism, and LFPS can suppress naturally occurring aggression. $A$, Experimental schedule for the effect of optical stimulation and pharmacological blockade on aggression. Left, Green area in the MeApv represents the viral spread. Blue stick represents the location of optical fibers. The effect of LFPS ( 900 pulses at $1 \mathrm{~Hz}$ ) was tested by applying LFPS immediately after HFPS, and then testing for aggression levels 30 min after the onset of HFPS. Mice were only stimulated once. $\boldsymbol{B}-\boldsymbol{E}$, HFPS primes aggression and is suppressed by LFPS delivered immediately after HFPS. Two-way ANOVA was used for statistical analysis across groups $(n=10$ ChR2 unstimulated, 10 ChR2 HFPS, 10 ChR2 HFPS + LFPS, 6 GFP unstimulated, 5 GFP HFPS, 6 GFP HFPS + LFPS mice). For (hR2 versus GFP: $F_{(1,41)}=12.839$ for $\boldsymbol{B}, 15.573$ for $\boldsymbol{C}, 17.772$ for $\boldsymbol{D}, 19.98$ for $\boldsymbol{E} ; p<0.001$ for $\boldsymbol{B}$ - . For photostimulated versus sham: $F_{(2,41)}=6.998$ for $\boldsymbol{B}, 6.737$ for $\boldsymbol{C}, 8.848$ for $\boldsymbol{D}, 7.818$ for $\boldsymbol{E} ; p=0.002$ for $\boldsymbol{B}, p=0.003$ for $\boldsymbol{C}, p<0.001$ for $\boldsymbol{D}, p<0.001$ for $\boldsymbol{E}$. For interaction between all groups: $F_{(2,41)}=6.687$ for $\boldsymbol{B}, 6.759$ for $\boldsymbol{C}, 4.442$ for $\boldsymbol{D}, 4.608$ for $\boldsymbol{E} ; p=0.003$ for $\boldsymbol{B}, p=0.003$ for $\boldsymbol{C}, p=0.018$ for $\boldsymbol{D}, p=0.016$ for $\boldsymbol{E}$. Holm-Sidak correction was used for multiple comparisons between groups. $\boldsymbol{F}-\boldsymbol{I}$, Inhibition of HFPS-induced aggression enhancement by the NMDAR blocker. Mice were administered systemic injections of MK-801 (100-300 $\mu \mathrm{g} / \mathrm{kg}) 30 \mathrm{~min}$ before HFPS and then tested for aggression $30 \mathrm{~min}$ later as above. Mice were only injected and stimulated once. One-way ANOVA was used for statistical analysis across groups ( $n=8$ unstimulated, 9 HFPS, 5 HFPS + $100 \mu \mathrm{g} / \mathrm{kg}$ MK-801, 5 HFPS + $150 \mu \mathrm{g} / \mathrm{kg}$ MK-801, 5 HFPS $+300 \mu \mathrm{g} / \mathrm{kg}$ MK-801 mice): $F_{(4,27)}=6$. R2648 for $\boldsymbol{F}, 6.683$ for $\boldsymbol{G}, 7.586$ for $\boldsymbol{H}, 21.961$ for $\boldsymbol{I} . p<0.001$ for all groups. Holm-Sidak correction was used for multiple comparisons between groups. J-M, LFPS suppresses naturally occurring aggressive behavior. Mice individually housed for 3 weeks to increase baseline aggression were delivered LFPS and $30 \mathrm{~min}$ after the onset of light were allowed to freely interact with a novel conspecific for $10 \mathrm{~min}$ ( $n=6 \mathrm{ChR2}, 7 \mathrm{GFP}$ mice). Two-tailed Student's $t$ tests were used to compare the same mouse before and after LFPS. For ChR2: $t_{(5)}=4.958$ for $\boldsymbol{J}, 2.936$ for $\boldsymbol{K}, 3.698$ for $\boldsymbol{L}, 5.136$ for $\boldsymbol{M} ; p=0.004$ for $\boldsymbol{J}, p=0.032$ for $\boldsymbol{K}, p=0.014$ for $\boldsymbol{L}, p=0.033$ for $\boldsymbol{M}$. For GFP: $t_{(7)}=0.546$ for $\boldsymbol{J}, 0.133$ for $\boldsymbol{K},-0.611$ for $\boldsymbol{L},-0.833$ for $\boldsymbol{M} ; p=0.605$ for $\boldsymbol{J}, p=0.898$ for $\boldsymbol{K}, p=0.564$ for $\boldsymbol{L}, p=0.437$ for $\boldsymbol{M}$. Data are mean \pm SEM. ${ }^{*} p<0.05$, ${ }^{* *} p<0.01,{ }^{* *} p<0.001$.

Since LFPS blocks aggression priming, we tested the effect of LFPS on HFPS-induced aggression increase (Fig. 5A). LFPS delivered immediately after HFPS reduced the effect of HFPS on attack behavior (Fig. 5B-E). Because $100 \mathrm{~Hz}$ stimulation induces NMDAR-dependent potentiation of synapses in various brain regions, including the MeA (Shindou et al., 1993; Watanabe et al., 1995, 1996; Smerin et al., 2016), we tested whether NMDARs are required for the effect of HFPS on aggression by intraperitoneally injecting the NMDAR antagonist MK-801 to virus- injected mice at $30 \mathrm{~min}$ before HFPS. MK-801 administered at 100,150 and $300 \mu \mathrm{g} / \mathrm{kg}$, doses shown to block NMDAR-dependent behavior (Zhou et al., 2017), blocked the effect of HFPS on aggression (Fig. 5F-I). Baseline aggression levels were comparable in mice administered HFPS and HFPS followed by LFPS [two-way ANOVA: related to Fig. $5 B, F_{(1,41)}=0.71, p=0.40$; related to Fig. $5 C, F_{(1,41)}=1.50, p=0.23$; related to Fig. $5 D$, $F_{(1,41)}=0.71, p=0.40$; related to Fig. $\left.5 E, F_{(1,41)}=0.02, p=0.90\right]$ or MK-801 before HFPS [one-way ANOVA: related to Fig. 5F, 

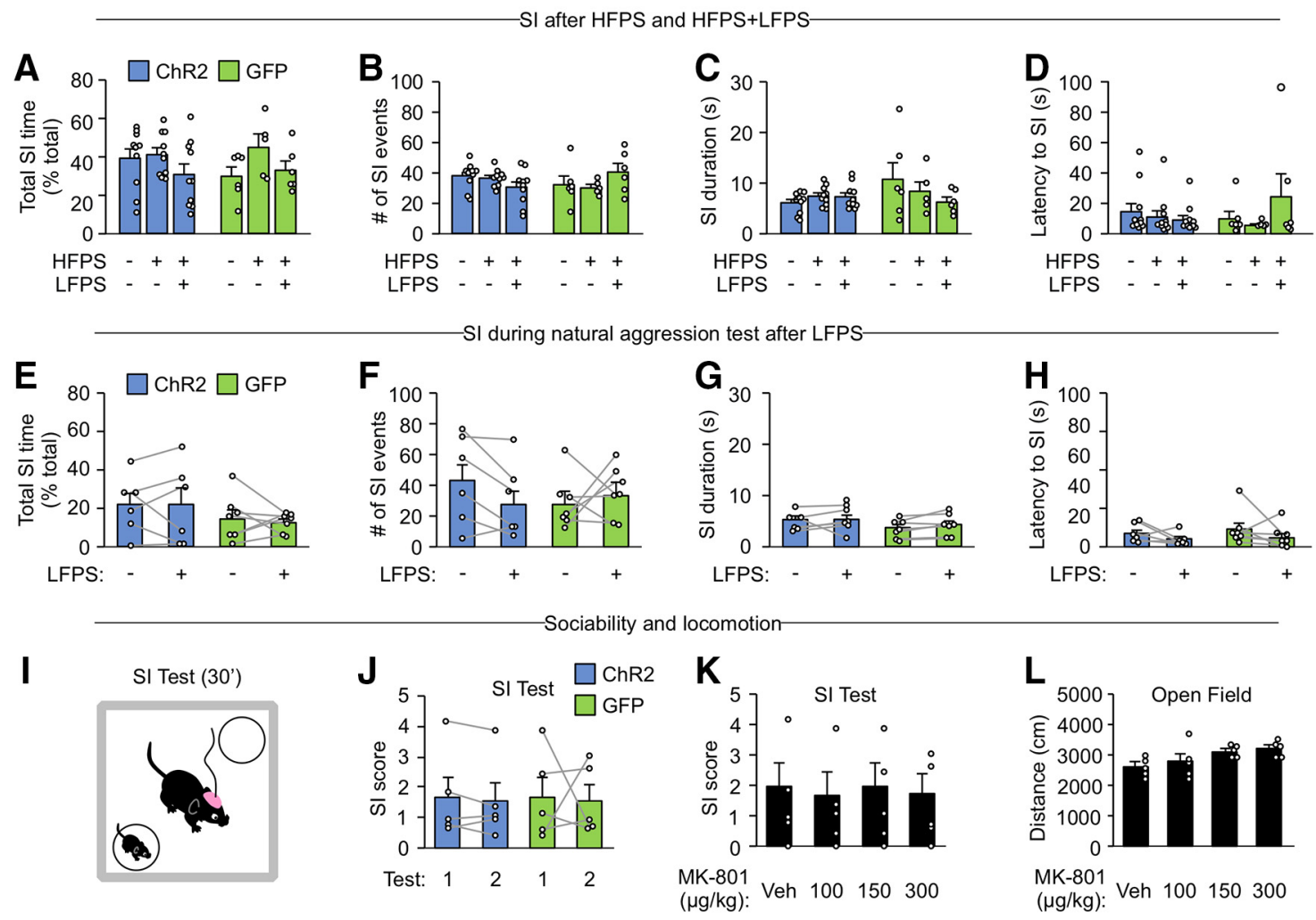

Figure 6. Photostimulation of MeApv neurons has no effect on nonaggressive social interaction. All mice were injected with ChR2 or GFP AAV 3 weeks before behavioral tests. $A-D$, Nonaggressive social interaction after HFPS or HFPS followed by LFPS ( $n=10$ ChR2 unstimulated, 10 ChR2 HFPS, 10 ChR2 HFPS + LFPS, 6 GFP unstimulated, 5 GFP HFPS, 6 GFP HFPS + LFPS mice). Two-way ANOVA was used for statistical analysis across groups. For ChR2 versus GFP: $F_{(1,41)}=0.065$ for $\boldsymbol{A}, 0.029$ for $\boldsymbol{B}, 1.731$ for $\boldsymbol{C}, 0.108$ for $\boldsymbol{D}$; $p=0.800$ for $\boldsymbol{A}, p=0.865$ for $\boldsymbol{B} ; p=0.196$ for $\boldsymbol{C} ; p=0.744$ for $\boldsymbol{D}$. For photostimulated versus sham: $F_{(2,41)}=2.277$ for $\boldsymbol{A}, 0.114$ for $\boldsymbol{B}, 0.748$ for $\boldsymbol{C}, 0.813$ for $\boldsymbol{D} ; p=0.115$ for $\boldsymbol{A}, p=0.893$ for $\boldsymbol{B}, p=0.480$ for $\boldsymbol{C}, p=0.450$ for $\boldsymbol{D}$. For interaction between all groups: $F_{(2,41)}=0.911$ for $\boldsymbol{A}, 3.008$ for $\boldsymbol{B}, 2.274$ for $\boldsymbol{C}, 1.639$ for $\boldsymbol{D} ; p=0.410$ for $\boldsymbol{A}, p=0.060$ for $\boldsymbol{B}, p=0.116$ for $\boldsymbol{C}, p=0.207$ for $\boldsymbol{D}$. Holm-Sidak correction was used for multiple comparisons between groups. $\boldsymbol{E}-\boldsymbol{H}$, Nonaggressive social interaction $1 \mathrm{~d}$ before and 30 min after LFPS ( $n=6$ ChR2 and 7 GFP mice). Two-tailed paired Student's $t$ tests were used to compare the same mouse before and after LFPS. For ChR2: $t_{(5)}=0.065$ for $\boldsymbol{E}_{,}-0.727$ for $\boldsymbol{F}$, 4.930 for $\boldsymbol{G}, 0.092$ for $\boldsymbol{H} ; p=0.976$ for $\boldsymbol{E}, p=0.446$ for $\boldsymbol{F}, p=0.154$ for $\boldsymbol{G}, p=0.970$ for $\boldsymbol{H}$. For GFP: $\boldsymbol{t}_{(7)}=0.496$ for $\boldsymbol{E},-0.903$ for $\boldsymbol{F},-1.643$ for $\boldsymbol{G},-0.196$ for $\boldsymbol{H} ; p=0.672$ for $\boldsymbol{E}, p=0.387$ for $\boldsymbol{F}, p=0.114$ for $\boldsymbol{G}, p=0.862$ for $\boldsymbol{H}$. $\boldsymbol{I}-\boldsymbol{K}$, Experimental protocol and quantification of $\mathrm{SI}$ score (the ratio of time spent exploring the cup with a mouse to that spent exploring the empty cup) during a sociability test starting at $30 \mathrm{~min}$ after HFPS $(\boldsymbol{J})$ or MK-801 injection $(\boldsymbol{K})$. J, ChR2 $(n=5)$ and GFP $(n=5)$ injected mice were tested on 2 consecutive days as with aggression testing. Two-tailed paired Student's $t$ test was used to compare test day 1 to test day 2: for (hR2, $t_{(4)}=0.934, p=0.403$; for GFP, $t_{(4)}=0.103, p=0.923 . K$, One-way ANOVA was used for statistical analysis across groups injected with vehicle $(n=4$ mice) or $100 \mu \mathrm{g} / \mathrm{kg} \mathrm{MK-801} \mathrm{(} n=4 \mathrm{mice}), 150 \mu \mathrm{g} / \mathrm{kg}$ MK801 ( $n=4$ mice), or $300 \mu \mathrm{g} / \mathrm{kg}$ MK-801 ( $n=4$ mice): $F_{(3,12)}=0.0321, p=0.992$. Holm-Sidak correction was used for multiple comparisons between groups. $L$, Analysis of the effect of MK-801 injection on distance traveled during an open field test ( $n=5$ mice for each group). One-way ANOVA was used for statistical analysis across groups: $F_{(3,16)}=2.838, p=0.071$. Holm-Sidak correction was used for multiple comparisons between groups. Data are mean \pm SEM.

$F_{(4,23)}=1.09, p=0.39$; related to Fig. $5 G, F_{(4,23)}=0.81, p=0.53$; related to Fig. $5 H, F_{(4,23)}=1.96, p=0.14$; related to Fig. 5I, $\left.F_{(4,23)}=2.58, p=0.064\right]$.

Mice with high baseline aggression may have already been primed and, therefore, are susceptible to LFPS. Indeed, prior winning experience enhances aggression and increases the probability of winning future contests (Valzelli, 1973; Flannelly et al., 1982; Potegal, 1992; Hsu et al., 2006; Stevenson and Rillich, 2012; Miczek et al., 2013; Hoopfer, 2016). To test this possibility, we applied LFPS to mice individually housed for 3 weeks to increase baseline aggression levels. LFPS delivered to the MeApv at $30 \mathrm{~min}$ before the aggression test inhibited aggressive behavior (Fig. 5J-M). These results indicate that LFPS suppresses natural aggression.

In contrast to attack behavior, nonaggressive social behaviors during the aggression test were unchanged by HFPS, HPFS+ LFPS, MK-801 injection, or natural aggression and were comparable to baseline levels (Fig. $6 A-H$ ), indicating that our optogenetic and pharmacological manipulations do not promote aggression by enhancing social interaction in general. To confirm this, we assessed sociability at $1 \mathrm{~d}$ before (Test 1) and
30 min after HFPS stimulation (Test 2) using a chamber with two wire cups: one empty and the other one containing a conspecific mouse (Fig. 6I). Mice naturally spend more time exploring the cup containing the conspecifics than the empty one, as indicated by a $>1$ social interaction score (SI, the ratio of time spent exploring the cup containing a mouse to that spent exploring the empty cup; Fig. $6 J$ ). The animal's preference for social subjects was not altered by HFPS stimulation or IP injections of MK-801 (Fig. 6J,K), indicating that NMDAR-dependent HFPS does not alter sociability. Additionally, MK-801 injection had no effect on locomotion in the open field test (Fig. 6L). These results are consistent with our finding that aggression priming has no effect on nonaggressive social interaction (Fig. $1 G-J$ ).

Together, these findings indicate that HFPS mimics aggression priming and therefore suggest a role for synaptic potentiation in the MeApv circuits in aggression priming.

The MeApv-VmH and MeApv-BNST pathways mediate the effect of HFPS on aggression

MeApv neurons project to several regions implicated in aggression, including the $\mathrm{VmH}, \mathrm{BNST}, \mathrm{MPO}$, and LS (Gomez and 

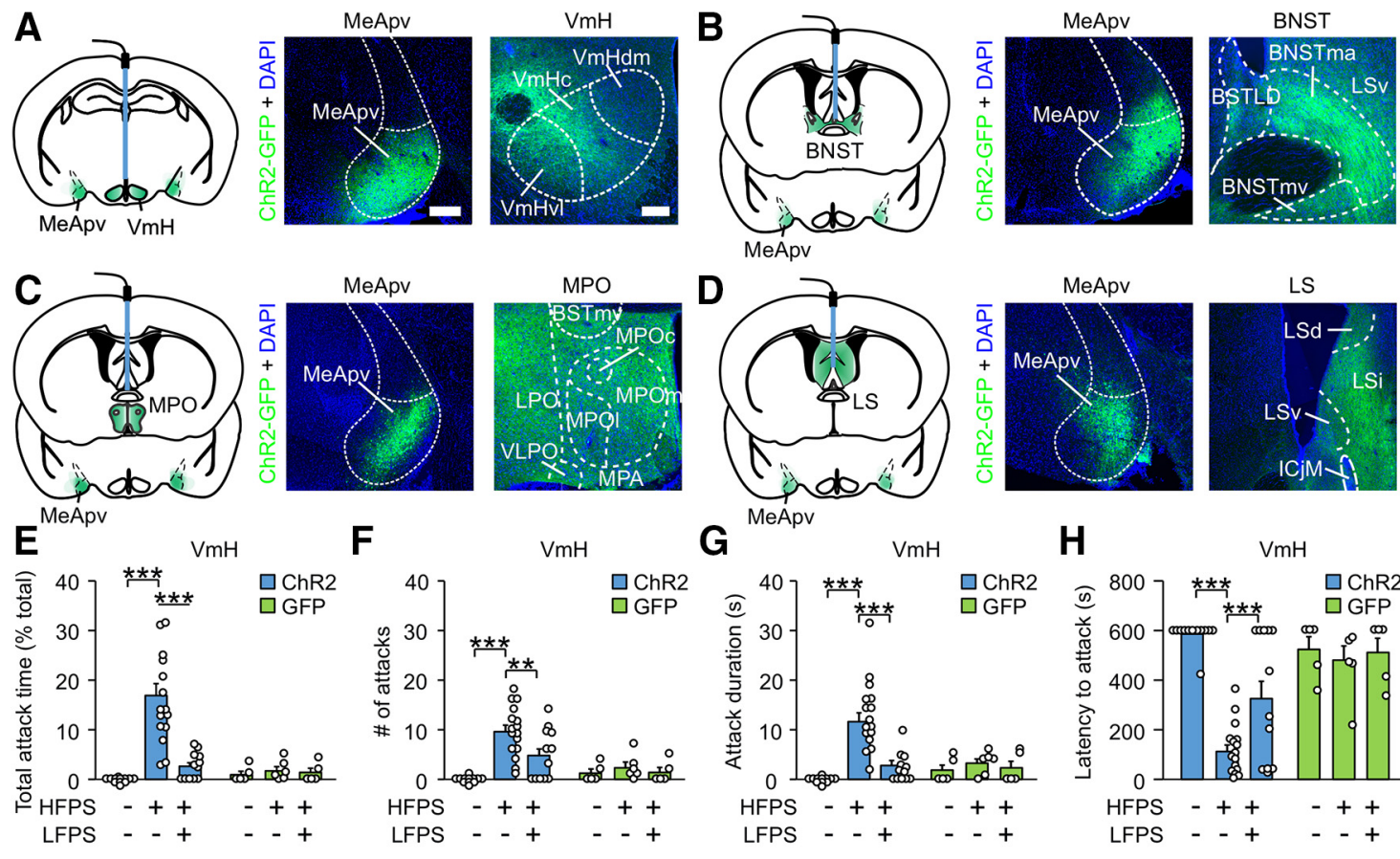

$\mathbf{F}$
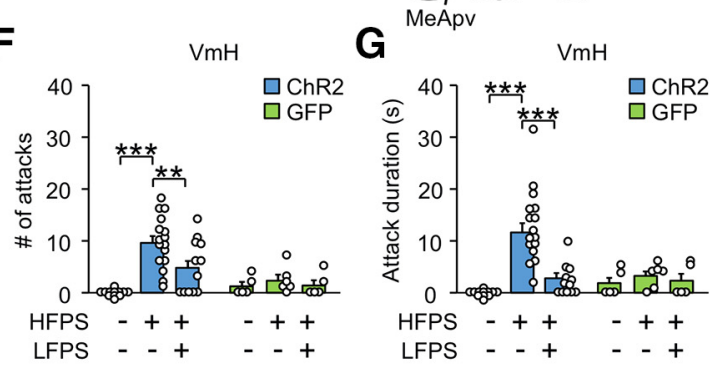

H
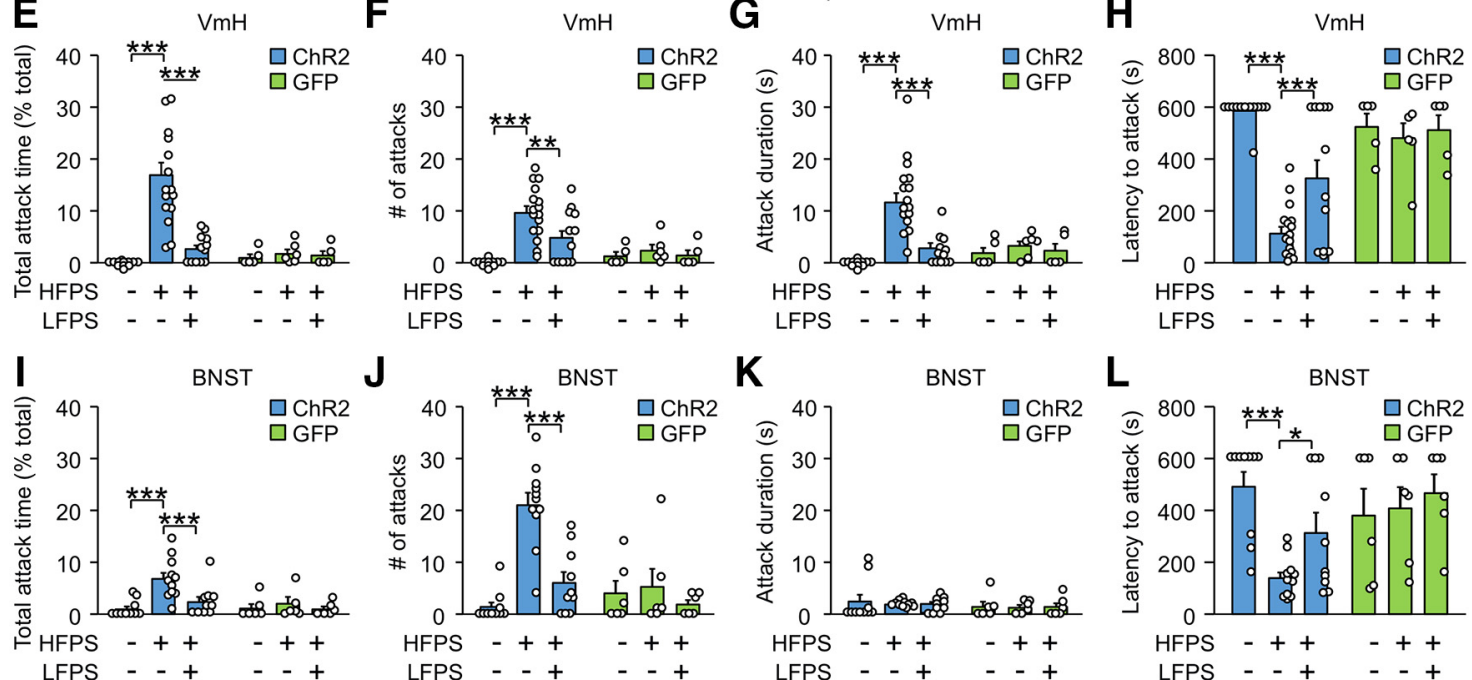

K
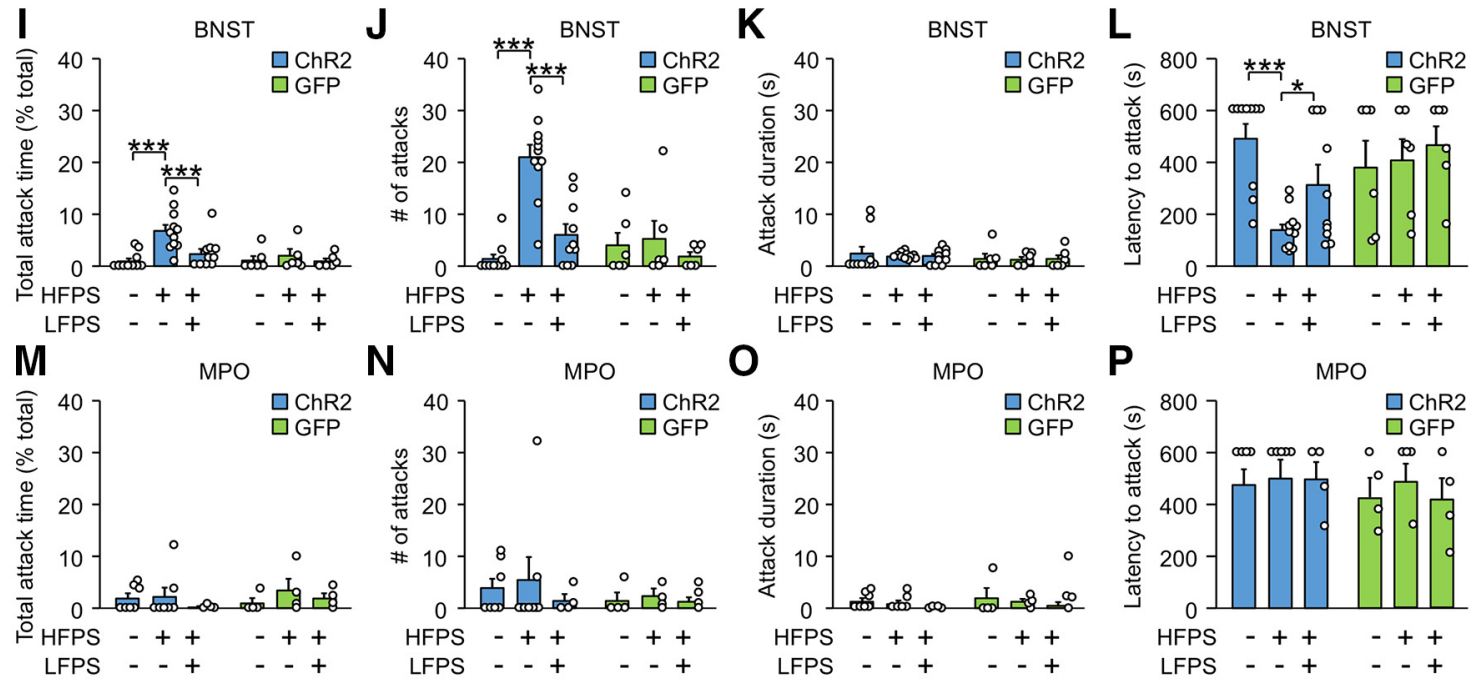

$\mathbf{N}$

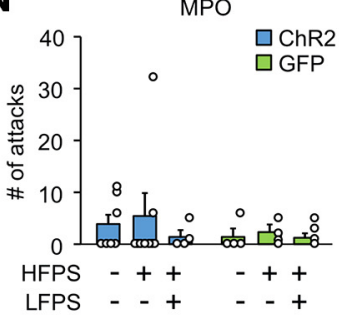

0
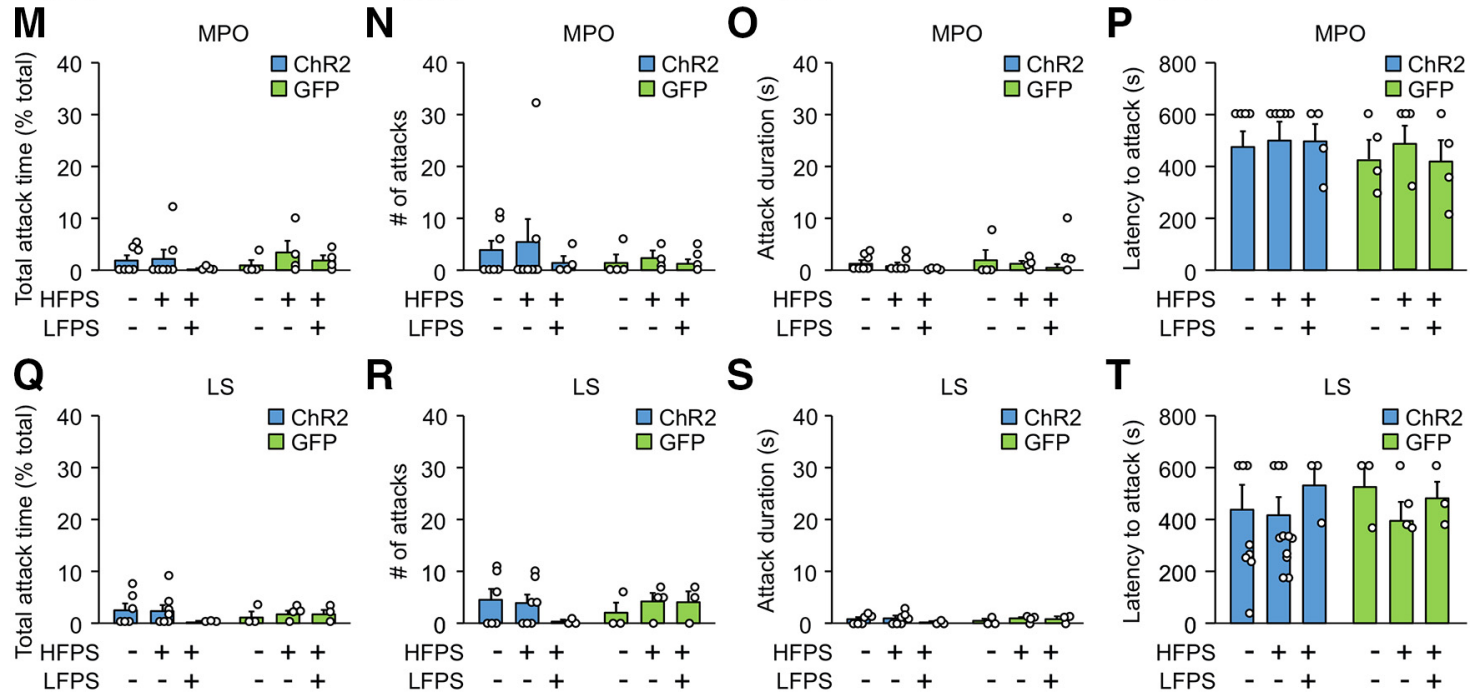

Figure 7. HFPS of projections from the MeApv to the VmH and BNST promotes aggression. Mice were pair-housed for 8 weeks after surgery, before stimulating projections from the MeApv to the VmH, BNST, MPO, or LS (HFPS alone or HFPS followed by LFPS). Optical stimulation was applied 30 min before introduction of conspecifics and each animal was only stimulated once. $\boldsymbol{A}-\boldsymbol{D}$, Illustrations for the viral spread (green areas) and optical fiber placement (blue sticks), and representative images of ChR2 expression at the MeApv and the projection areas of MeApv neurons. Scale bars: $\boldsymbol{A}(\boldsymbol{A}-\boldsymbol{D})$ left, $1 \mathrm{~mm}$; right, $100 \mu \mathrm{m}$. $\boldsymbol{E}-\boldsymbol{H}$, The effect of HFPS and HFPS + LFPS at the VmH on aggressive behavior $(n=12$ ChR2 unstimulated, 16 ChR2 HFPS, 13 ChR2 HFPS + LFPS, 5 GFP unstimulated, 6 ChR2 HFPS, 5 ChR2 HFPS + LFPS mice). Two-way ANOVA was used for statistical analysis across groups. For (hR2 versus GFP: $F_{(1,52)}=11.466$ for $\boldsymbol{E}$, 9.567 for $\boldsymbol{F}, 4.982$ for $\boldsymbol{G}, 5.043$ for $\boldsymbol{H} ; p<0.001$ for $\boldsymbol{E}, p=0.003$ for $\boldsymbol{F}, p=0.030$ for $\boldsymbol{G}, p=0.029$ for $\boldsymbol{H}$. For sham versus stimulated mice: $\boldsymbol{F}_{(2,52)}=13.311$ for $\boldsymbol{E}$, $F_{(2,52)}=9.552$ for $\boldsymbol{F}$, $F_{(2,52)}=13.627$ for $\boldsymbol{G}, F_{(2,52)}=5.333$ for $\boldsymbol{H} ; p<0.001$ for $\boldsymbol{E}, p<0.001$ for $\boldsymbol{F}, p<0.001$ for $\boldsymbol{G}, p=0.008$ for $\boldsymbol{H}$. For interaction between all groups: $F_{(2,52)}=10.851$ for $\boldsymbol{E}, 5.1$ for $\boldsymbol{F}, 7.962$ for $\boldsymbol{G}$, 13.082 for $\boldsymbol{H} ; p<0.001$ for $\boldsymbol{E}, p=0.009$ for $\boldsymbol{F}, p<0.001$ for $\boldsymbol{G}, p<0.001$ for $\boldsymbol{H}$. Holm-Sidak was used for post hoc multiple comparisons between groups. $\boldsymbol{I}-\boldsymbol{L}$, The effect of HFPS and HFPS + LFPS at the BNST on aggressive behavior ( $n=10$ ChR2 unstimulated, 11 ChR2 HFPS, 10 ChR2 HFPS + LFPS, 6 GFP unstimulated, 6 GFP HFPS, 6 GFP HFPS + LFPS mice). Two-way ANOVA was used for statistical analysis across groups. For ChR2 versus GFP: $F_{(1,43)}=6.997$ for $\boldsymbol{I}, 16.99$ for $\boldsymbol{J}, 2.414$ for $\boldsymbol{K}, 0.247$ for $\boldsymbol{L} ; p=0.011$ for $\boldsymbol{I}, p<0.001$ for $\boldsymbol{J}, p=0.128$ for $\boldsymbol{K}, p=0.622$ for $\boldsymbol{L}$. For sham versus stimulated mice: $F_{(2,43)}=5.718$ for $I, 18.861$ for $J, 0.227$ for $\boldsymbol{K}, 3.271$ for $\boldsymbol{L} ; p=0.006$ for $\boldsymbol{I}, p<0.001$ for $J, p=0.798$ for $\boldsymbol{K}, p=0.048$ for $\boldsymbol{L}$. For interaction between all groups: $F_{(2,43)}=3.798$ for $\boldsymbol{I}, 6.73$ for $\boldsymbol{J}, 0.737$ for $\boldsymbol{K}, 23.402$ for $\boldsymbol{L} ; p=0.03$ for $\boldsymbol{I}, p=0.003$ for $\boldsymbol{J}, p=0.484$ for $\boldsymbol{K}, p<0.001$ for $\boldsymbol{L}$. Holm-Sidak was used for post hoc multiple comparisons between two groups. $\boldsymbol{M - P}$, The effect of HFPS and HFPS + LFPS at the MPO on aggressive behavior ( $n=7$ ChR2 unstimulated, 7 ChR2 HFPS, 4 ChR2 HFPS + LFPS, 4 GFP unstimulated, 4 GFP HFPS, 4 GFP 
Newman, 1992; Canteras et al., 1995; Coolen and Wood, 1998; Haller et al., 2006; Nelson and Trainor, 2007; Hashikawa et al., 2016). To locate the downstream target regions of the MeApv involved in aggression priming, we tested whether HFPS of the MeApv projections to these areas can increase aggression. To this end, we injected ChR2 or GFP virus into the MeApv and implanted optical fibers in the $\mathrm{VmH}$, BNST, MPO, or LS for stimulation of MeApv axons. At 2 months after injection, viral expression in the MeApv and the terminal fields of MeApv neurons at the VmH, BNST, MPO, and LS was readily detected (Fig. $7 A-D)$. It is noted that the MeApv axons were dense in the medial portion of the BNST and in the VmHvl and central portion of the $\mathrm{VmH}$, areas implicated in aggression (Fuxjager et al., 2010; Carrillo et al., 2011; Lin et al., 2011; Lee et al., 2014; Masugi-Tokita et al., 2016).

On the test day, HFPS was delivered to the VmH, BNST, $\mathrm{MPO}$, or LS at $30 \mathrm{~min}$ before the aggression test. Baseline aggression levels were comparable for each group [two-way ANOVA: related to Fig. $7 E, F_{(1,49)}=0.01, p=0.92$; related to Fig. $7 F$, $F_{(1,49)}=0.35, p=0.56$; related to Fig. $7 G, F_{(1,49)}=0.01, p=0.92$; related to Fig. $7 H, F_{(1,49)}=0.83, p=0.37$; related to Fig. $7 I$, $F_{(1,43)}=0.01, p=0.91$; related to Fig. $7 J, F_{(1,43)}=0.05, p=0.82$; related to Fig. $7 K, F_{(1,43)}=0.01, p=0.94$; related to Fig. $7 L$, $F_{(1,43)}=0.04, p=0.84$; related to Fig. $7 M, F_{(1,44)}=1.01, p=0.33$; related to Fig. $7 N, F_{(1,44)}=0.38, p=0.55$; related to Fig. $7 O$, $F_{(1,44)}=0.26, p=0.62$; related to Fig. $7 P, F_{(1,44)}=0.34, p=0.57$; related to Fig. $7 Q, F_{(1,22)}=0.01, p=0.94$; related to Fig. $7 R$, $F_{(1,22)}=0.07, p=0.79$; related to Fig. $7 S, F_{(1,22)}=0.01, p=0.91$; related to Fig. $\left.7 T, F_{(1,22)}=0.03, p=0.86\right]$. HFPS increased aggression from baseline and unstimulated controls when applied to the VmH and BNST, but not to the MPO or LS (Fig. 7E-T). The increase was suppressed by LFPS delivered to the same region immediately after HFPS (Fig. $7 E-L$ ), indicative of the involvement of synaptic potentiation in HFPS-induced aggression escalation. HFPS and HFPS + LFPS at the VmH, BNST, MPO, or LS had no effect on nonaggressive social behaviors (Fig. 8A-T), and therefore did not alter general social interaction.

It is noted that HFPS of MeApv projections at the $\mathrm{VmH}$ increased both attack number and duration per attack, whereas stimulation of MeApv projections to the BNST had a robust effect on attack number but not attack duration (Fig. $7 F, G, J, K$ ). Consistent with this finding, total attack time was increased more by stimulation in the $\mathrm{VmH}$ than in the BNST (Fig. 7E,I).

\section{$\leftarrow$}

HFPS + LFPS mice). Two-way ANOVA was used for statistical analysis across groups. For ChR2 versus GFP: $F_{(1,24)}=0.263$ for $\boldsymbol{M}, 0.439$ for $\boldsymbol{N}, 1.506$ for $\boldsymbol{0}, 0.271$ for $\boldsymbol{P} ; \boldsymbol{p}=0.613$ for $\boldsymbol{M}, p=0.514$ for $\boldsymbol{N}, p=0.232$ for $\boldsymbol{0}, p=0.607$ for $\boldsymbol{P}$. For sham versus stimulated mice: $F_{(2,24)}=0.611$ for $\boldsymbol{M}, 0.128$ for $\boldsymbol{N}, 0.425$ for $\boldsymbol{0}, 0.476$ for $\boldsymbol{P} ; p=0.551$ for $\boldsymbol{M}, p=0.881$ for $\boldsymbol{N}$, $p=0.659$ for $\boldsymbol{0}, p=0.627$ for $\boldsymbol{P}$. For interaction between all groups: $F_{(2,24)}=0.677$ for $\boldsymbol{M}$, 0.273 for $\boldsymbol{N}, 1.306$ for $\boldsymbol{0}, 0.300$ for $\boldsymbol{P} ; \boldsymbol{p}=0.518$ for $\boldsymbol{M}, p=0.764$ for $\boldsymbol{N}, \boldsymbol{p}=0.289$ for $\boldsymbol{0}$, $p=0.744$ for $\boldsymbol{P}$. Holm-Sidak was used for post hoc multiple comparisons between two groups. Q-T, The effect of HFPS and HFPS + LFPS at the LS on aggressive behavior $(n=6$ ChR2 unstimulated, 7 ChR2 HFPS, 3 ChR2 HFPS + LFPS, 3 GFP unstimulated, 4 GFP HFPS, 3 GFP HFPS + LFPS mice). Two-way ANOVA was used for statistical analysis across groups. For ChR2 versus GFP: $F_{(1,20)}=0.108$ for $\mathbf{Q}, 0.0001$ for $\boldsymbol{R}, 0.0122$ for $S, 0.383$ for $\boldsymbol{T} ; p=0.745$ for $\mathbf{Q}, p=0.991$ for $\boldsymbol{R}, p=0.913$ for $\boldsymbol{S}, p=0.543$ for $\boldsymbol{T}$. For sham versus stimulated mice: $F_{(2,20)}=0.470$ for $\boldsymbol{Q}, 0.574$ for $\boldsymbol{R}, 0.852$ for $\boldsymbol{S}, 0.628$ for $\boldsymbol{T} ; p=0.632$ for $\boldsymbol{Q}, p=0.573$ for $\boldsymbol{R}$, $p=0.442$ for $\boldsymbol{S}, p=0.544$ for $\boldsymbol{T}$. For interaction between all groups: $F_{(2,20)}=0.725$ for $\mathbf{Q}$, 1.067 for $\boldsymbol{R}, 0.721$ for $\boldsymbol{S}, 0.476$ for $\boldsymbol{T} ; p=0.497$ for $\boldsymbol{Q}, p=0.363$ for $\boldsymbol{R}, p=0.498$ for $\boldsymbol{S}$, $p=0.628$ for $T$. Holm-Sidak was used for post hoc multiple comparisons between two groups. Data are mean \pm SEM. ${ }^{*} p<0.05,{ }^{* *} p<0.01,{ }^{* * *} p<0.001$.
To confirm that direct light stimulation of MeApv projections to the $\mathrm{VmH}$ and BNST activates $\mathrm{VmH}$ and BNST neurons, we recorded VmH and BNST neurons using whole-cell patch-clamp in brain slices from mice injected with the ChR2 virus at the MeApv (Fig. 8U,V). We recorded $20 \mathrm{VmH}$ and 24 BNST neurons that fired action potentials in response to injection of depolarizing currents and were hyperpolarized by injection of hyperpolarizing currents (Fig. $8 \mathrm{~W}, \mathrm{Y}$ ). Of them, $17 \mathrm{VmH}$ neurons and 20 BNST neurons showed light-evoked EPSPs and EPSCs in response to photostimulation (Fig. $8 X, Z$ ). Hence, photostimulation of MeApv axons locally at the $\mathrm{VmH}$ and BNST can elicit postsynaptic responses.

To determine whether MeApv projections to the $\mathrm{VmH}$ and BNST are indeed responsible for the aggression-promoting effect of HFPS at the MeApv, we inactivated the MeApv projections to the $\mathrm{VmH}$ or BNST during HFPS of the MeApv. To this end, we injected a combination of AAV-expressing ChR2E123A fused to mCherry, ArchT fused to YFP (both under control of the CaMKII $\alpha$ promoter), or GFP virus into the MeApv, and placed optical fibers into the MeApv and either the $\mathrm{VmH}$ or BNST in the same animal (Fig. 9A). The expression of ChR2-mCherry and ArchT-YFP in the MeApv projections to the $\mathrm{VmH}$ and BNST was readily detected at 8 weeks after viral injection (Fig. $9 B, C)$.

To confirm that photostimulation of ArchT inhibits neuronal activity, we used whole-cell patch-clamp to record MeApv neurons from brain slices of mice injected with ArchT virus. The $7 \mathrm{~s}$ depolarizing currents were injected into MeApv neurons expressing ArchT, and $561 \mathrm{~nm}$ light pulses were delivered during the third second of current injection (Fig. 9D). Current injection induced action potentials (Fig. 9D). This was suppressed during light stimulation and resumed after termination of light (Fig. 9D), indicating that optical stimulation of ArchT can inhibit MeApv neurons. Moreover, direct stimulation of ArchT expressed by MeApv axons in the VmH and BNST abolished spiking of $\mathrm{VmH}$ and BNST neurons induced by current injection (Fig. 9E,F). Hence, stimulation of ArchT in axons effectively inhibits synaptic transmission.

For aggression testing, ChR2 and ArchT-injected mice were tested for baseline aggression and $1 \mathrm{~d}$ later delivered HFPS at the MeApv while simultaneously inhibiting MeApv projections to the VmH or BNST using overlapping $1 \mathrm{~s}$ pulses of $561 \mathrm{~nm}$ light during each train of HFPS (Fig. 9A,G,H). Aggression was tested $30 \mathrm{~min}$ later. HFPS of the MeApv alone increased attack duration and total attack number, while decreasing the latency to first attack (Fig. 9I-P), as shown above (Fig. 5B-E). Inhibition of the MeApv-VmH projections blocked the effect of MeApv HFPS on total attack time, attack duration, and latency to the first attack (Fig. 9I,K,L), indicating that these projections are required for HFPS of the MeApv to modulate these parameters of attack. The number of attacks in the MeApv-VmH inhibition group was lower than in the MeApv HFPS group, but the difference did not reach statistical significance (Fig. 9J). This is likely because, although HFPS of the MeApv-VmH synapse does not induce attacks, it extends naturally occurring attack bouts that are otherwise too short to be detected, and inhibition of these synapses abolishes this effect. It appears that the MeApv-VmH pathway mainly modulates attack duration. Inactivating the MeApvBNST projections abolished all the effects of direct stimulation of the MeApv on aggression (Fig. 9M-P), presumably because of the blockade of attack initiation, which is controlled by the MeApv-BNST projections (Fig. 7J). Baseline aggression [oneway ANOVA: related to Fig. $9 I, F_{(3,30)}=0.36, p=0.85$; related to 

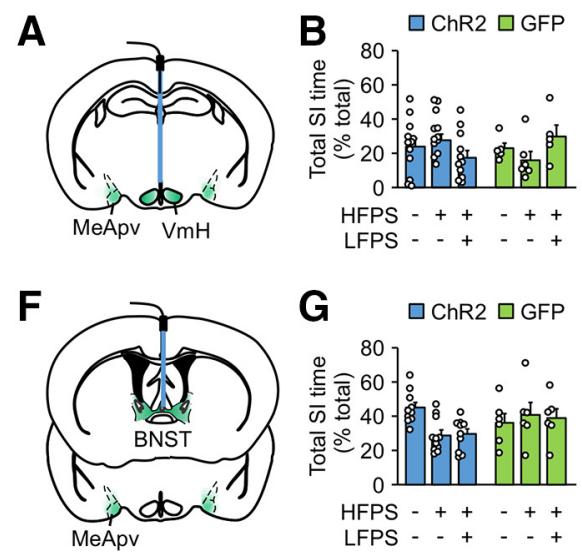

G $\quad \square \mathrm{ChR2} \square \mathrm{GFP}$
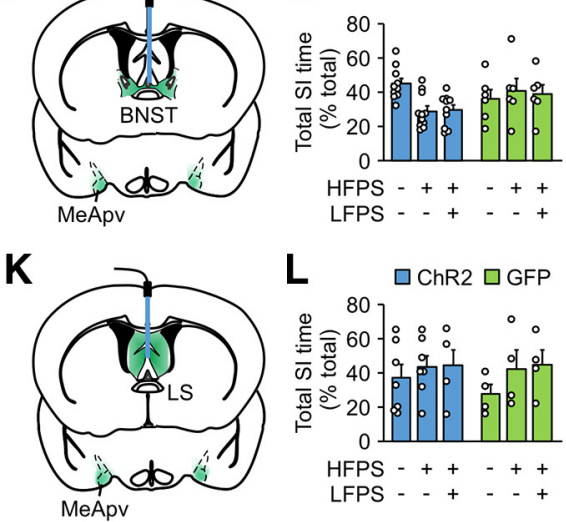

L $\quad$ C ChR2 $\square$ GFP
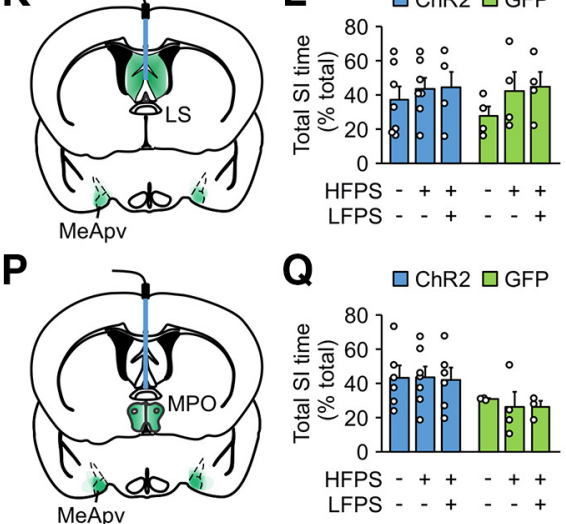

U

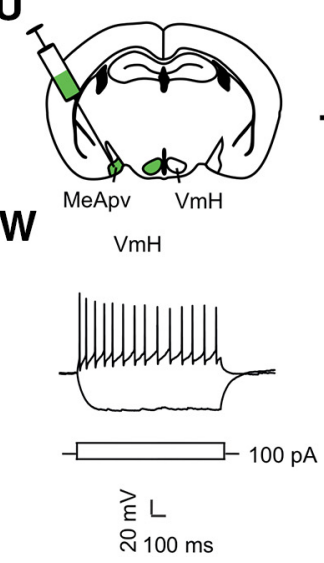

Q $\square$ ChR2 $\square$ GFP

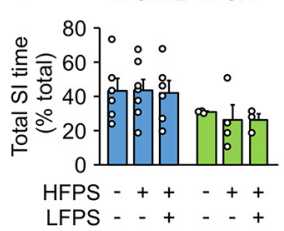

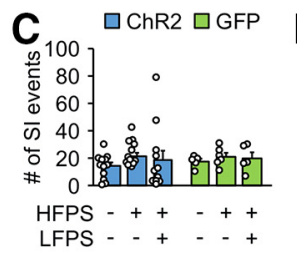

H $\quad$ ChR2 $\square$ GFP
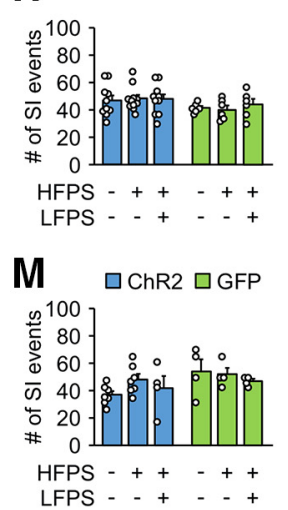

R IChR2 IGFP

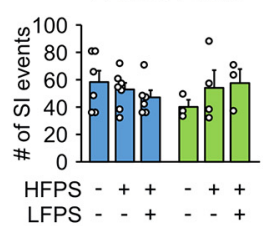

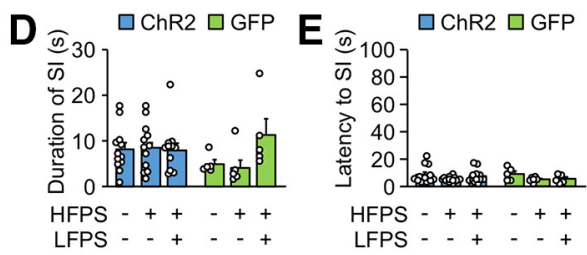
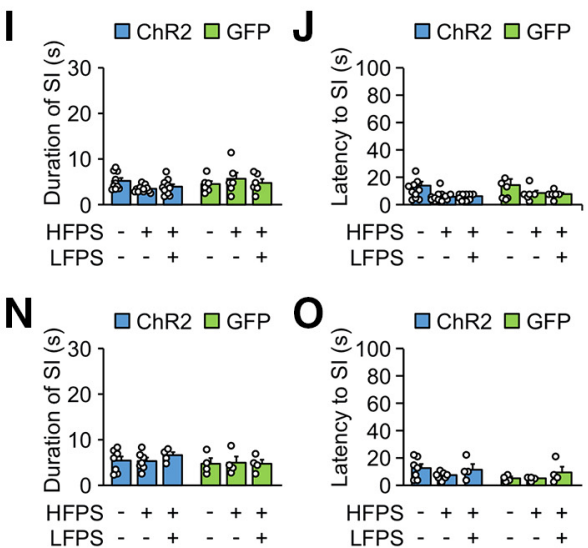

S $\square$ ChR2 $\square$ GFP $\mathbf{T}$

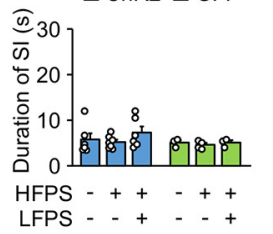

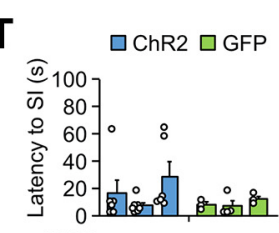

HFPS - + + + + LFPS - - + - +

\section{V}

$\mathbf{X}$

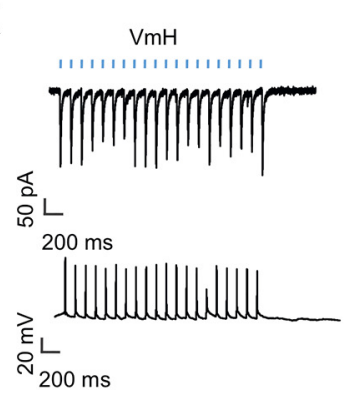

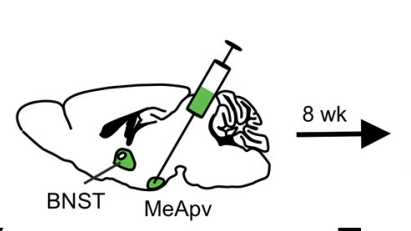

Z

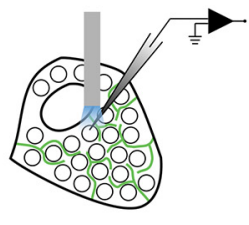

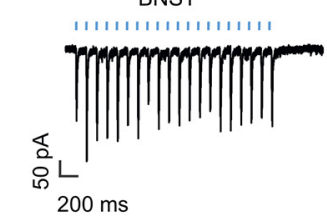

हे ᄂ

尺े $100 \mathrm{~ms}$
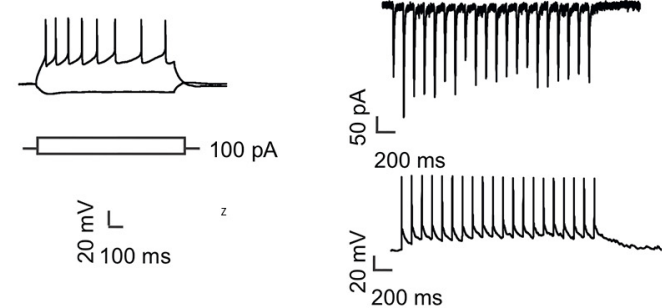

Figure 8. Optogenetic stimulation of MeApv projection areas depolarizes the VmH and BNST neurons and has no effect on nonaggressive social interaction. $\boldsymbol{A}, \boldsymbol{F}, \boldsymbol{K}, \boldsymbol{P}$, Illustrations for the viral spread (green areas) and optical fiber placement (blue sticks). $\boldsymbol{B}-\boldsymbol{E}, \mathbf{G}-\mathbf{J}, \mathbf{L}-\mathbf{O}, \mathbf{Q}-\boldsymbol{T}$, Analysis of nonaggressive social interaction during the 10 min aggression test. $\boldsymbol{B}-\boldsymbol{E}$, The effect of HFPS and HFPS + LFPS at the VmH on aggressive behavior ( $n=12$ ChR2 unstimulated, 16 ChR2 HFPS, 13 ChR2 HFPS + LFPS, 5 GFP unstimulated, 6 ChR2 HFPS, 5 ChR2 HFPS + LFPS mice). Two-way ANOVA was used for statistical analysis across groups. For ChR2 versus GFP: $F_{(1,52)}=0.270$ for $\boldsymbol{B}, 0.002$ for $\boldsymbol{C}, 1.741$ for $\boldsymbol{D}, 1.143$ for $\boldsymbol{E} ; p=0.606$ for $\boldsymbol{B}, p=0.963$ for $\boldsymbol{C}, p=0.193$ for $\boldsymbol{D}$, $p=0.290$ for $\boldsymbol{E}$. For sham versus stimulated mice: $F_{(2,52)}=0.242$ for $\boldsymbol{B}, 1.615$ for $\boldsymbol{C}, 2.610$ for $\boldsymbol{D}, 1.435$ for $\boldsymbol{E} ; p=0.786$ for $\boldsymbol{B}, p=0.209$ for $\boldsymbol{C}, p=0.083$ for $\boldsymbol{D}, p=0.247$ for $\boldsymbol{E}$. For interaction between all groups: $F_{(2,52)}=3.020$ for $\boldsymbol{B}, 0.046$ for $\boldsymbol{C}, 3.116$ for $\boldsymbol{D}, 0.309$ for $\boldsymbol{E} ; p=0.057$ for $\boldsymbol{B}, p=0.955$ for $\boldsymbol{C}, p=0.053$ for $\boldsymbol{D}, p=0.735$ for $\boldsymbol{E}$. Holm-Sidak was used for post hoc multiple comparisons between groups. $G-J$, The effect of HFPS and HFPS + LFPS at the BNST on aggressive behavior ( $n=10$ ChR2 unstimulated, 11 ChR2 HFPS, 10 ChR2 HFPS + LFPS, 6 GFP unstimulated, 6 GFP HFPS, 6 GFP HFPS + LFPS mice). Two-way ANOVA was used for statistical analysis across groups. For ChR2 versus GFP: $F_{(1,43)}=1.420$ for $\boldsymbol{G}, 3.978$ for $\boldsymbol{H}, 1.624$ for $\boldsymbol{I}, 0.780$ for $\boldsymbol{J}$; $p=0.240$ for $\boldsymbol{G}, p=0.052$ for $\boldsymbol{H}, p=0.209$ for $\boldsymbol{I}, p=0.382$ for $\boldsymbol{J}$. For sham versus stimulated mice: $F_{(2,43)}=1.407$ for $\mathbf{G}, 0.165$ for $\boldsymbol{H}, 0.298$ for $\boldsymbol{I}, 3.214$ for $\boldsymbol{J} ; p=0.256$ for $\boldsymbol{G}, p=0.848$ for $\boldsymbol{H}$, $p=0.744$ for $\boldsymbol{I}, p=0.052$ for $\boldsymbol{J}$. For interaction between all groups: $F_{(2,43)}=3.567$ for $\mathbf{G}, 0.150$ for $\boldsymbol{H}, 2.156$ for $\boldsymbol{I}, 0.133$ for $\boldsymbol{J} ; p=0.037$ for $\boldsymbol{G}, p=0.861$ for $\boldsymbol{H}, p=0.128$ for $\boldsymbol{I}, p=0.876$ for $\boldsymbol{J}$. Holm-Sidak was used for post hoc multiple comparisons between two groups. L-0, The effect of HFPS and HFPS + LFPS at the MPO on aggressive behavior ( $n=7$ ChR2 unstimulated, 7 ChR2 HFPS, 4 ChR2 HFPS + LFPS, 4 GFP unstimulated, 4 GFP HFPS, 4 GFP HFPS + LFPS mice). Two-way ANOVA was used for statistical analysis across groups. For ChR2 versus GFP: $F_{(1,24)}=0.212$ for $\boldsymbol{L}, 4.039$ for $\boldsymbol{M}, 1.288$ for $\boldsymbol{N}, 3.254$ for $\mathbf{0} ; p=0.649$ for $\boldsymbol{L}, p=0.056$ for $\boldsymbol{M}, p=0.268$ for $\boldsymbol{N}, p=0.084$ for $\boldsymbol{0}$. For sham versus stimulated mice: $F_{(2,24)}=1.158$ for $\boldsymbol{L}, 0.622$ for $\boldsymbol{M}, 0.192$ for $\boldsymbol{N}, 1.417$ for $\boldsymbol{0} ; p=0.331$ for $\boldsymbol{L}, p=0.545$ for $\boldsymbol{M}, p=0.827$ for $\boldsymbol{N}, p=0.262$ for $\boldsymbol{0}$. For interaction between all groups: $F_{(2,24)}=0.206$ for $\boldsymbol{L}, 0.995$ for $\boldsymbol{M}, 0.286$ for $\boldsymbol{N}, 0.656$ for $\mathbf{0}$; $p=0.816$ for $\boldsymbol{L}, p=0.384$ for $\boldsymbol{M}, p=0.754$ for $\boldsymbol{N}, p=0.528$ for $\mathbf{0}$. Holm-Sidak was used for post hoc multiple comparisons between two groups. Q- $\boldsymbol{T}$, The effect of HFPS and HFPS + LFPS at the LS on aggressive behavior ( $n=6$ ChR2 unstimulated, 7 ChR2 HFPS, 3 ChR2 HFPS + LFPS, 3 GFP unstimulated, 4 GFP HFPS, 3 GFP HFPS + LFPS mice). Two-way ANOVA was used for statistical analysis across groups. For ChR2 versus GFP: $F_{(1,20)}=2.578$ for $\mathbf{Q}, 0.336$ for $\boldsymbol{R}, 0.378$ for $\boldsymbol{S}, 0.591$ for $\boldsymbol{T} ; p=0.124$ for $\boldsymbol{Q}, p=0.569$ for $\boldsymbol{R}, p=0.545$ for $\boldsymbol{S}, p=0.451$ for $\boldsymbol{T}$. For sham versus stimulated mice: $F_{(2,20)}=0.155$ for $\boldsymbol{Q}, 0.471$ for $\boldsymbol{R}, 0.382$ for $S, 1.207$ for $\boldsymbol{T} ; p=0.857$ for $\boldsymbol{Q}, p=0.631$ for $\boldsymbol{R}, p=0.688$ for $\boldsymbol{S}, p=0.32$ for $\boldsymbol{T}$. For interaction between all groups: $F_{(2,20)}=0.153$ 
Fig. 9J, $F_{(3,30)}=0.69, p=0.57$; related to Fig. $9 K, F_{(3,30)}=0.09$, $p=0.96$; related to Fig. $9 L, F_{(3,30)}=0.13, p=0.94$; related to Fig. $9 M, F_{(3,33)}=0.75, p=0.53$; related to Fig. $9 N, F_{(3,33)}=0.01$, $p=1.00$; related to Fig. $9 O, F_{(3,33)}=0.84, p=0.48$; related to Fig. $\left.9 P, F_{(3,33)}=0.03, p=0.99\right]$ and nonaggressive social behaviors were unchanged during baseline testing [one-way ANOVA: related to Fig. 9Q, $F_{(3,33)}=2.72, p=0.06$; related to Fig. $9 R$, $F_{(3,33)}=0.90, p=0.46$; related to Fig. $9 S, F_{(3,33)}=0.65, p=0.59$; related to Fig. $9 T, F_{(3,31)}=0.86, p=0.47$; related to Fig. $9 U$, $F_{(3,33)}=0.30, p=0.82$; related to Fig. $9 V, F_{(3,33)}=0.53, p=0.67$; related to Fig. $9 W, F_{(3,33)}=0.23, p=0.87$; related to Fig. $9 X$, $\left.F_{(3,33)}=0.85, p=0.48\right]$ and after stimulation (Fig. 9Q-X). These results indicate that the $\mathrm{MeApv}-\mathrm{VmH}$ and MeApv-BNST pathways are necessary for HFPS of the MeApv to promote aggression.

Together, these findings indicate that HFPS of the MeApv enhances aggression through the MeApv-VmH and MeApvBNST synapses, and that the MeApv-VmH and MeApv-BNST pathways regulate different stages of attack behavior.

\section{HFPS and aggressive behavior induce potentiation of the MeApv-VmH and MeApv-BNST synapses}

To confirm that the MeApv-VmH and MeApv-BNST synapses can indeed undergo synaptic potentiation, we prepared brain slices containing $\mathrm{VmH}$ and BNST from mice injected with ChR2 virus at the MeApv and recorded EPSCs induced by light pulses with whole-cell patch-clamp. HFPS (four trains of 50, $2 \mathrm{~ms}$ pulses at $100 \mathrm{~Hz}$ ) increased EPSCs in both $\mathrm{VmH}$ and BNST neurons (Fig. 10A,B), indicating that synaptic potentiation is induced.

Next, we tested whether HFPS of the MeApv potentiates $\mathrm{MeApv}-\mathrm{VmH}$ and MeApv-BNST synapses in vivo by recording optically evoked LFPs at the VmH or BNST. To this end, we injected ChR2 virus into the MeApv and 6 weeks later implanted a microdrive-controlled optrode consisting of 16 tungsten wires and an optical fiber into the VmH or BNST. Only mice expressing the virus and with an optrode in the target region determined post hoc were included in the analysis of fEPSPs (Fig. 10C,D).

To test the effect of HFPS on synaptic strength, LFPs evoked by $473 \mathrm{~nm}$ light stimulation at $0.05 \mathrm{~Hz}$ were recorded before and after HFPS or HFPS followed by LFPS. fEPSPs were analyzed as previously demonstrated (Xiong et al., 2015; Zhou et al., 2017). HFPS increased light-evoked fEPSPs at the VmH and at the BNST (Fig. 10E,F). These effects were blocked by LFPS applied immediately after HFPS and by MK-801 (150 $\mu \mathrm{g} / \mathrm{kg}$, i.p.) injected at $30 \mathrm{~min}$ before HFPS (Fig. 10E,F). Hence, HFPS of the MeApv potentiates the MeApv-VmH and MeApv-BNST synapses via NMDARs.

To test whether these same synapses are potentiated by attack experience, we recorded LFPs evoked by photostimulation of the MeApv projections at the $\mathrm{VmH}$ or BNST before and after

\footnotetext{
$\leftarrow$

for $\boldsymbol{Q}, 0.598$ for $\boldsymbol{R}, 0.215$ for $\boldsymbol{S}, 0.437$ for $\boldsymbol{T} ; p=0.859$ for $\boldsymbol{Q}, p=0.56$ for $\boldsymbol{R}, p=0.808$ for $\boldsymbol{S}$, $p=0.652$ for $\boldsymbol{T}$. Holm-Sidak was used for post hoc multiple comparisons between two groups. $\boldsymbol{U}-\boldsymbol{Z}$, Six- to 7-week-old mice were injected with ChR2 AAV into the MeApv. Brain slices containing the VmH $(\boldsymbol{W}, X)$ or BNST $(Y, Z)$ were prepared from injected mice 8 weeks later. Neurons in brain slices were recorded with whole-cell patch-clamp. $\boldsymbol{W}$, Responses of $\mathrm{VmH}$ neurons to injection of $1 \mathrm{~s}$ depolarizing and hyperpolarizing currents. $\boldsymbol{X}$, EPSCs and EPSPs induced by $2 \mathrm{~ms}, 15 \mathrm{mw} / \mathrm{mm}^{2} 473 \mathrm{~nm}$ light pulses delivered at $10 \mathrm{~Hz}$ to $\mathrm{VmH}$ neurons. $Y$, Responses of BNST neurons to injection of $1 \mathrm{~s}$ depolarizing and hyperpolarizing currents. Z, EPSCs and EPSPs induced by $2 \mathrm{~ms}, 15 \mathrm{mw} / \mathrm{mm}^{2} 473 \mathrm{~nm}$ light pulses delivered at $10 \mathrm{~Hz}$ to BNST neurons. Data are mean \pm SEM.
}

aggression testing. To this end, virally injected and optrodeimplanted mice were individually housed to enhance aggression. On the test day, a mouse bearing an optrode was placed in a test cage and recorded for optically evoked LFPs at $0.05 \mathrm{~Hz}$ as above. After 30 min of baseline recording, an unimplanted target mouse was placed into the test cage for $10 \mathrm{~min}$. Light-evoked fEPSPs were recorded throughout and after the interaction period.

The recorded mice were divided into two groups according to whether they attacked (AGG) or not (NON) during the interaction period. fEPSPs recorded after placement of the target mouse were aligned to the onset of either the first attack (for AGG mice) or the first social interaction event (for NON mice) across mice (Fig. 10G,H). In AGG mice, fEPSP recorded at the $\mathrm{VmH}$ and BNST transiently increased after attack (Fig. 10G,H). By contrast, fEPSP recorded at the $\mathrm{VmH}$ and BNST before and after the interaction period were unchanged in NON mice (Fig. 10G, $H$ ). To assess whether LFPS, which inhibits aggression priming, has an effect on attack-induced potentiation of MeApv-VmH and MeApv-BNST synapses, we applied LFPS immediately upon removal of the target mouse. LFPS abolished attackinduced potentiation of both MeApv-VmH and MeApv-BNST synapses (Fig. 10G,H). These results indicate that attacking a conspecific induces synaptic potentiation.

Together, these findings show that both HFPS and attack behavior potentiate the MeApv-VmH and MeApv-BNST synapses.

\section{Traumatic stress enhances attack behavior through potentiation of the MeApv aggression circuit}

In addition to agonistic encounters, traumatic experience can have a significant impact on aggression in both animals and humans. In humans, traumatic experience can elevate the rate of aggressive behavior and aggression may be a symptom of PTSD (Taft et al., 2012; American Psychiatric Association, 2013). In rodents, traumatic stress induced by electric foot shock increases aggression (Pynoos et al., 1996; Olson et al., 2011). To test whether the MeApv is involved in aggression related to traumatic-stress, we adopted a protocol that uses electric foot shock to induce stress-enhanced fear learning (SEFL, a PTSD-associated behavior) in rats (Rau et al., 2005). We first tested whether this protocol can induce SEFL in mice. Male mice ( 9 weeks of age) with low baseline aggression levels were used. On the day of traumatic stress induction (day 1), mice were placed in a chamber (Context A) to receive foot shocks over the course of a 90 min session. After day 1, mice were divided into two groups to test for SEFL (Fig. 11A): one group received one shock in a new context (Context B) and were tested for freezing $1 \mathrm{~d}$ after (Context B test); the other group was tested for freezing behavior in Context A without shock for 30 min for 4 consecutive days (extinction test); 1, 10, and 15 foot shocks all increased freezing during the Context B test (nontraumatic context; Fig. 11B), indicating that fear learning is enhanced. During the $4 \mathrm{~d}$ extinction test, mice given 1 or 10 foot shocks decreased freezing more pronouncedly than those receiving 15 shocks (Fig. 11C). We next tested the effect of foot shock on aggression. Fifteen shocks were delivered to the mice as it induced stronger SEFL than 1 and 10 shocks. These mice became more aggressive after foot shock than controls that had been placed in the shock box but not shocked (Fig. 11D-G).

Since foot shock increases aggression, we assessed the effect of foot shock on the MeApv-VmH and MeApv-BNST synapses with in vivo recording. Mice (6 weeks of age, male) were injected with the ChR2 virus at the MeApv and implanted with a 16- 
A ChR2.mCherry, ArchT.YFP
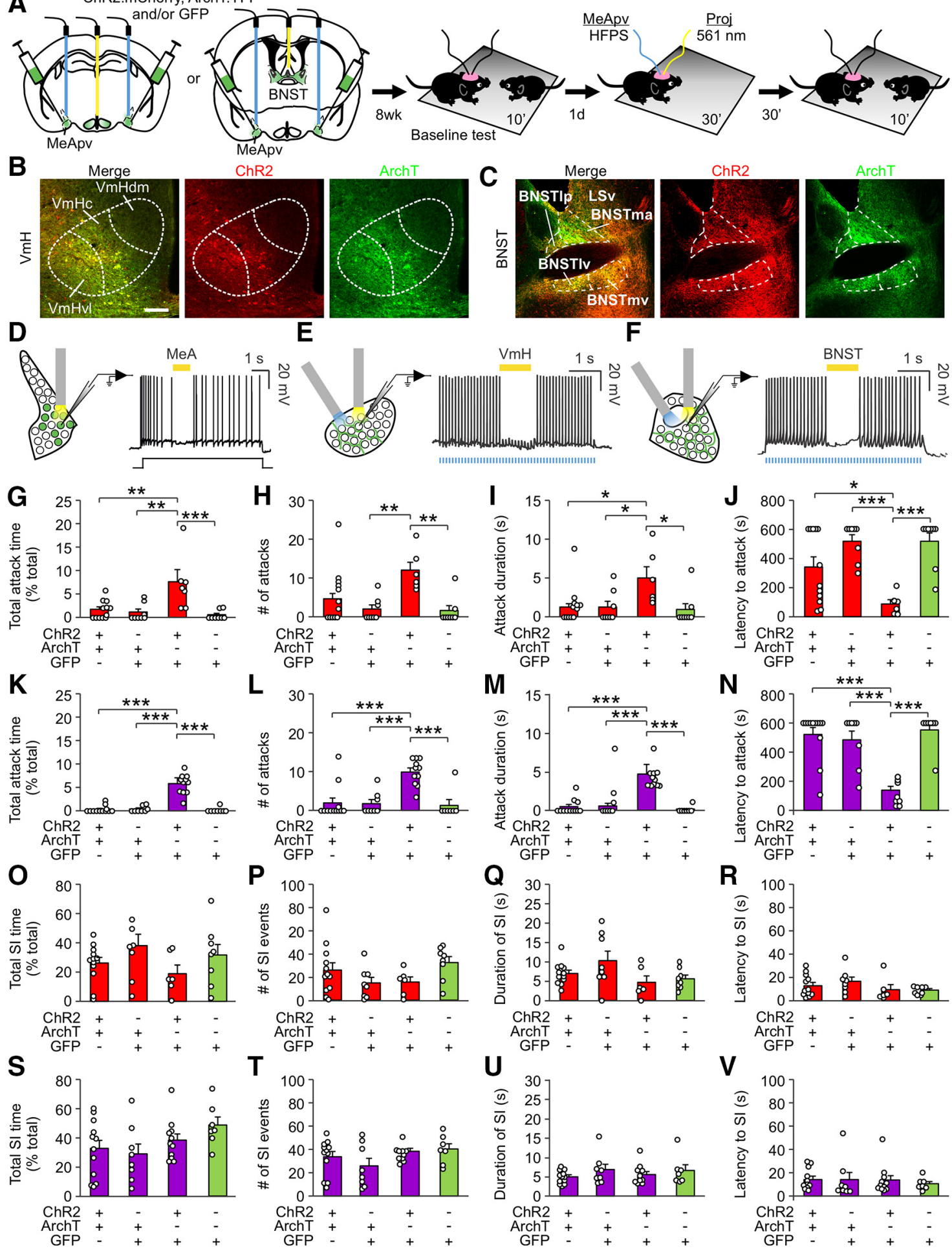

Figure 9. MeApv projections to the VmH and BNST are required for HFPS at the MeApv to enhance aggression. $A$, Experimental schedule. Six- to -7-week-old mice were injected with AAV into the MeApv and then pair-housed for 8 weeks before electrophysiological recordings $(\boldsymbol{D}-\boldsymbol{F})$ or aggression testing $(\mathbf{G}-\boldsymbol{X})$. Mice were given HFPS at the MeApv and $561 \mathrm{~nm}$ light pulses $(1 \mathrm{~s}$ per pulse, overlapping with each train of HFPS) at the VmH or BNST, 30 min before aggression testing. Left, Green area in the MeApv represents the viral spread. Blue stick represents the location of optical fibers. $\boldsymbol{B}, \boldsymbol{C}$, Representative images of ChR2E123A-mCherry and ArchT-GFP terminal expression at the VmH (B) and BNST (C). Scale bars: $\boldsymbol{B}, \boldsymbol{C}, 100 \mu \mathrm{m}$. D-F, Illustrations of sites of recordings and optical stimulation and representative traces of suppression of action potentials in MeApv, VmH, and BNST neurons. D, MeApv neurons were delivered $1 \mathrm{~s}, 15 \mathrm{mw} / \mathrm{mm}^{2} 561$ nм light pulses during current injection. $\boldsymbol{E}, \boldsymbol{F}$, EPSPs from VmH $(\boldsymbol{E})$ and BNST $(\boldsymbol{F})$ neurons were induced by $2 \mathrm{~ms}, 15 \mathrm{mw} / \mathrm{mm}^{2} 473 \mathrm{~nm}$ light pulses delivered at $10 \mathrm{~Hz}$ with an overlapping light pulse of $1 \mathrm{~s}, 15 \mathrm{mw} / \mathrm{mm}^{2}$, and $561 \mathrm{~nm}$. G-J, The effect of HFPS at the MeApv and optogenetic inhibition at VmH on aggressive behavior. One-way ANOVA was used for statistical analysis across groups: $F_{(3,30)}=7.454$ for $\boldsymbol{G}, 5.449$ for $\boldsymbol{H}, 3.780$ for $\boldsymbol{I}, 8.537$ for $\boldsymbol{J} ; p<0.001$ for $\boldsymbol{G}, p=0.004$ for $\boldsymbol{H}, p=0.021$ for $\boldsymbol{I}, p<0.001$ for $\boldsymbol{J} ; n=12$ (ChR2 + ArchT), 8 (GFP + ArchT), 6 (ChR2 + GFP), 8 (GFP) mice. Holm-Sidak was used for post hoc multiple comparisons between two groups. $\boldsymbol{K}-\boldsymbol{N}$, The effect of HFPS at the MeApv and optogenetic inhibition at BNST on aggressive behavior. One-way ANOVA was used for statistical analysis across groups: $F_{(3,34)}=22.597$ for $\boldsymbol{K}, 19.508$ for $\boldsymbol{L}, 12.582$ for $\boldsymbol{M}, 10.126$ for $\boldsymbol{N} ; p<0.001$ for $\boldsymbol{K}, p<0.001$ for $\boldsymbol{L}$, $p<0.001$ for $M, p<0.001$ for $N ; n=12$ (ChR2 + ArchT), 8 (GFP + ArchT), 11 (ChR2 + GFP), 7 (GFP) mice. Holm-Sidak was used for post hoc multiple comparisons between two groups. $\mathbf{0}-\boldsymbol{R}$, The effect of HFPS at the MeApv and optogenetic inhibition at VmH on nonaggressive social behavior. One-way ANOVA was used for statistical analysis across groups: $F_{(3,30)}=1.509$ for 
channel optrode into the VmH or BNST 6 weeks later (Fig. $11 H$ ). Light-evoked fEPSPs were recorded at the $\mathrm{VmH}$ and BNST before and after 15 foot shocks. fEPSPs increased after foot shock (Fig. 11I,J), indicating that foot shock induces potentiation of the MeApv-VmH and MeApv-BNST synapses.

Given that foot shock enhances aggression and induces potentiation of the MeApv-VmH and MeApv-BNST synapses (Fig. 11D-J), we tested whether LFPS of the MeApv circuits could curb aggression elevation after traumatic stress. We again injected mice (6 weeks of age) with ChR2 or GFP virus and implanted optical fibers into the MeApv (Fig. 12A). Injected mice received no or 15 foot shocks at 3 weeks after surgery. Foot shock-induced SEFL in injected mice was confirmed by contextual fear memory and fear memory extinction tests performed after shock (Fig. 12B,C).

To apply LFPS, mice were removed from the shock box immediately after shock, placed into novel cages, and allowed to acclimate for $10 \mathrm{~min}$ before delivery of LFPS to the MeApv. LFPS abolished the effect of foot shock on aggression in mice injected with ChR2, but not GFP virus (Fig. 12D-G). Notably, LFPS did not alter SEFL, nonaggressive social interaction behavior during the aggression test, sociability in the two-cup social interaction test, or locomotion in the open field test (Fig. $12 \mathrm{H}-\mathrm{N}$ ).

Together, these findings indicate that traumatic stress induces potentiation of the MeApv-VmH and MeApv-BNST synapses and that LFPS of the MeApv can block traumatic stress-induced increased aggression.

\section{Discussion}

Aggression is greatly influenced by experience. Prior agonistic or stressful experience can be a predictor for aggression symptoms associated with psychiatric disorders, such as PTSD and intermittent explosive disorder (Nelson and Trainor, 2007; Van Voorhees et al., 2016; Taft et al., 2017). The neural mechanism underlying experience-dependent modification of aggression, however, remains largely unclear. In this study, using a combination of optogenetic stimulation, in vivo electrophysiology, and behavioral assays, we found that the strength of synaptic transmission between the MeApv and $\mathrm{VmH}$ and BNST is increased by attack experience and traumatic stress. This synaptic potentiation underlies attack and trauma-induced aggression increase. These findings identify a role for synaptic plasticity within MeApv pathways in experience-dependent modulation of aggression.

\section{Potentiation of MeApv-VmH and MeApv-BNST synapses underlie experience-dependent modification of aggressive behavior}

Attack priming, or aggression priming, represents a form of natural, social experience-dependent modulation of aggression. We studied aggression priming in a novel arena rather than using

\section{$\leftarrow$}

$\mathbf{0}, 1.200$ for $\boldsymbol{P}, 1.992$ for $\boldsymbol{Q}, 2.403$ for $\boldsymbol{R} ; p=0.232$ for $\boldsymbol{0}, p=0.327$ for $\boldsymbol{P}, p=0.136$ for $\boldsymbol{Q}$, $p=0.087$ for $\boldsymbol{R} ; n=12$ (ChR2 + ArchT), 8 (GFP + ArchT), 6 (ChR2 + GFP), 8 (GFP) mice. Holm-Sidak was used for post hoc multiple comparisons between two groups. $\mathbf{S}-\boldsymbol{V}$, The effect of HFPS at the MeApv and optogenetic inhibition at BNST on nonaggressive social behavior. One-way ANOVA was used for statistical analysis across groups: $F_{(3,34)}=1.908$ for $S, 0.182$ for $\boldsymbol{T}, 1.682$ for $\boldsymbol{U}, 0.853$ for $\boldsymbol{V} ; p=0.147$ for $\boldsymbol{S}, p=0.908$ for $\boldsymbol{T}, p=0.189$ for $\boldsymbol{U}$, $p=0.475$ for $\boldsymbol{V} ; n=12$ (ChR2 + ArchT), 8 (GFP + ArchT), 11 (ChR2 + GFP), 7 (GFP) mice. Holm-Sidak was used for post hoc multiple comparisons between two groups. Data are mean \pm SEM. ${ }^{*} p<0.05,{ }^{* *} p<0.01,{ }^{* * *} p<0.001$. the resident intruder assay to minimize the effect of prior experience on aggression. In our "priming" study, mice that engaged in an aggressive, but not nonaggressive, social interaction with a conspecific became significantly more aggressive after the first interaction period. Mice exhibited predominantly offensive aggressive behavior, as most of the attacks are self-initiated with stereotyped behaviors associated with offensive aggression (e.g., lateral/rear attacks, chasing, and wrestling) (Blanchard and Blanchard, 1977, 2003b; Miczek and O’Donnell, 1978; Blanchard et al., 1979). Since high-frequency electrical stimulation at the MeApv mimics attack priming (Potegal et al., 1996a), synaptic potentiation may be a mechanism for the priming effect. We tested this possibility by applying LFPS, which has been widely used to reduce synaptic strength, to the MeA immediately after an aggressive encounter with a conspecific. We chose CaMKII $\alpha^{+}$ neurons because histologic analysis revealed that they are the major type of cells activated at the MeApv after "priming." LFPS at the MeApv blocked the effect of priming on aggressive behavior, supporting that synaptic potentiation is involved in aggression priming.

To directly measure synaptic potentiation, it is necessary to know the exact synapses where the potentiation occurs. We addressed this question by priming aggression with $100 \mathrm{~Hz}$ HFPS, which allows us to systematically screen MeApv target areas that may mediate aggression priming; $100 \mathrm{~Hz}$ HFPS, which has been used to successfully induce synaptic potentiation in vivo (Nabavi et al., 2014; Zhou et al., 2017), primes aggression in subject mice as attacking experience. Other frequencies we tested did not have such an effect, consistent with the earlier reports that $20 \mathrm{~Hz}$ optogenetic stimulation of glutamatergic MeA neurons does not trigger aggression and that $100 \mathrm{~Hz}$ stimulations are more effective in inducing synaptic potentiation than $40 \mathrm{~Hz}$ stimulations (Chen et al., 1996b; Hong et al., 2014). The behavioral effect of $100 \mathrm{~Hz}$ stimulation, therefore, must have arisen from the unique physiological changes it induces.

Our electrophysiological recording shows that MeApv neurons exhibit membrane depolarization locked to each stimulation. Since postsynaptic depolarization is necessary for synaptic potentiation (Golding et al., 2002; Remy and Spruston, 2007), and previous in vivo and ex vivo studies have shown that $100 \mathrm{~Hz}$ stimulation is an effective protocol for inducing synaptic potentiation (Bliss and Collingridge, 1993; Chen et al., 1996a; Nguyen and Kandel, 1997; Grover et al., 2009; Nabavi et al., 2014), the priming effect of $100 \mathrm{~Hz}$ HFPS suggests a potential role of synaptic potentiation in aggression priming. This possibility is consistent with our finding that LFPS and the NMDAR blocker MK801 suppress HFPS-induced enhancement of aggression. Nonaggressive social interaction, sociability, and locomotion were unchanged by $100 \mathrm{~Hz}$ HFPS, suggesting that the increase in aggression is not caused by alteration of general arousal or social interaction. It is noted that our findings do not exclude the possibility that other firing frequencies of MeA neurons during attack priming also induce synaptic potentiation. Indeed, since the $\mathrm{VmH}$ and BNST neurons are also activated by attacking behavior, and the pairing of low-frequency presynaptic firing with postsynaptic depolarization is an effective protocol of inducing synaptic potentiation, potentiation of the MeApv-VmH and MeApv-BNST synapses during aggression priming is likely induced by firing of MeApv neurons at lower frequencies. However, 20 and $40 \mathrm{~Hz}$ light stimulations without being paired with postsynaptic depolarization cannot induce synaptic potentiation as we demonstrated.

Having demonstrated that HFPS mimics aggression priming by attack, we optogenetically stimulated MeApv axons at various 

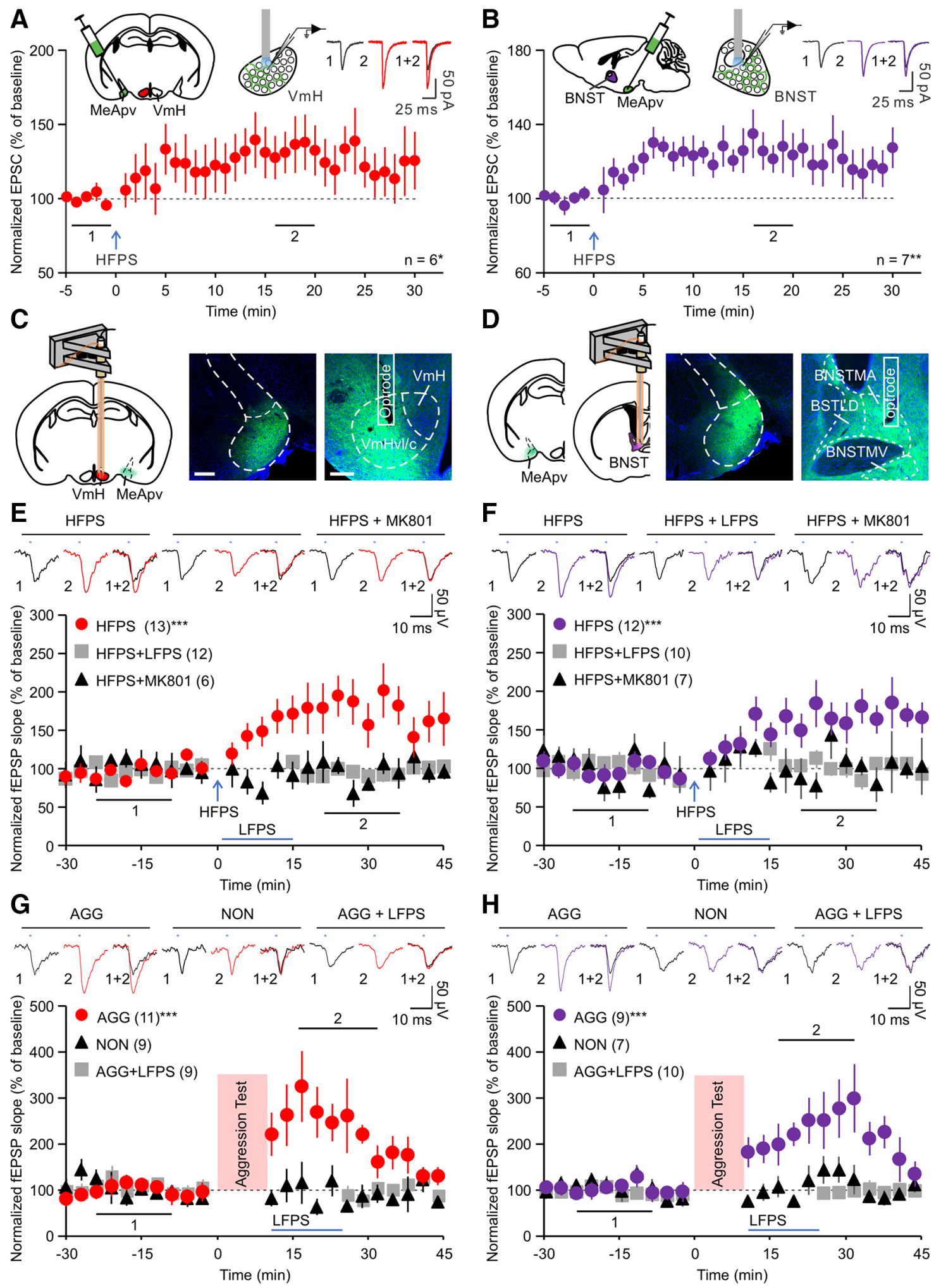

Figure 10. Attack priming and HFPS potentiate MeApv-VmH and MeApv-BNST synapses. $\boldsymbol{A}, \boldsymbol{B}$, EPSCs evoked by light pulses were recorded at the VmH $(\boldsymbol{A})$ or BNST (B) in brain slices from mice injected with virus in the MeApv; $n$ indicates the number of slices (one slice per animal). Repeated-measures ANOVA was used for statistical analysis across time points under the same experimental condition. $F_{(5,34)}=1.644, p<0.022$ for $\boldsymbol{A} ; F_{(6,34)}=2.008, p=0.002$ for $\boldsymbol{B}$. Holm-Sidak was used for post hoc multiple comparisons between two groups. $\boldsymbol{C}-\boldsymbol{H}$, ChR2 virus was injected into the MeApv, and optrodes were implanted into the VmH or BNST 6 weeks later and 2 weeks before recording. LFPs were evoked by stimulating at the VmH or BNST with 1 ms, $0.05 \mathrm{~Hz}$ light pulses before and after HFPS or a 10 min aggression test. $\boldsymbol{C}, \boldsymbol{D}$, Illustrations for the viral spread (green areas) and optrode placement (pink bars), and representative images of ChR2 expression at the MeApv (middle) and projections of MeApv neurons at the VmH (C) or BNST (D) (right). Optrode tract is indicated in images. Scale bars: $\boldsymbol{C}$, left, 2 mm; $\boldsymbol{C}$, right, $\boldsymbol{D}$, $200 \mu \mathrm{m} . \boldsymbol{E}, \boldsymbol{F}$, Normalized slopes of light-evoked fEPSPs recorded at the $\mathrm{VmH}(\boldsymbol{E})$ and BNST $(\boldsymbol{F})$ before and after light stimulation. MK-801 (150 $\mu \mathrm{g} / \mathrm{kg})$ was injected before the onset of recording. Insets, Representative traces. Each data point represents the average slope of the late component of 9 evoked fEPSPs. Repeated-measures ANOVA was used for statistical analysis across time points under the same experimental condition. For fEPSPs at the VmH: HFPS, $F_{(31,403)}=4.236, p<0.001 ;$ HFPS + LFPS, $F_{(31,341)}=0.907, p=0.614 ;$ HFPS + MK-801, $F_{(31,155)}=0.772$, $p=0.799$. Two-tailed paired Student's $t$ test was used to identify time points significantly different from baseline. For fEPSPs at the BNST: HFPS, $F_{(31,341)}=3.788, p<0.001 ;$ HFPS + LFPS, 
A

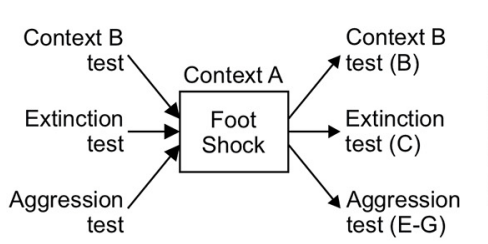

B
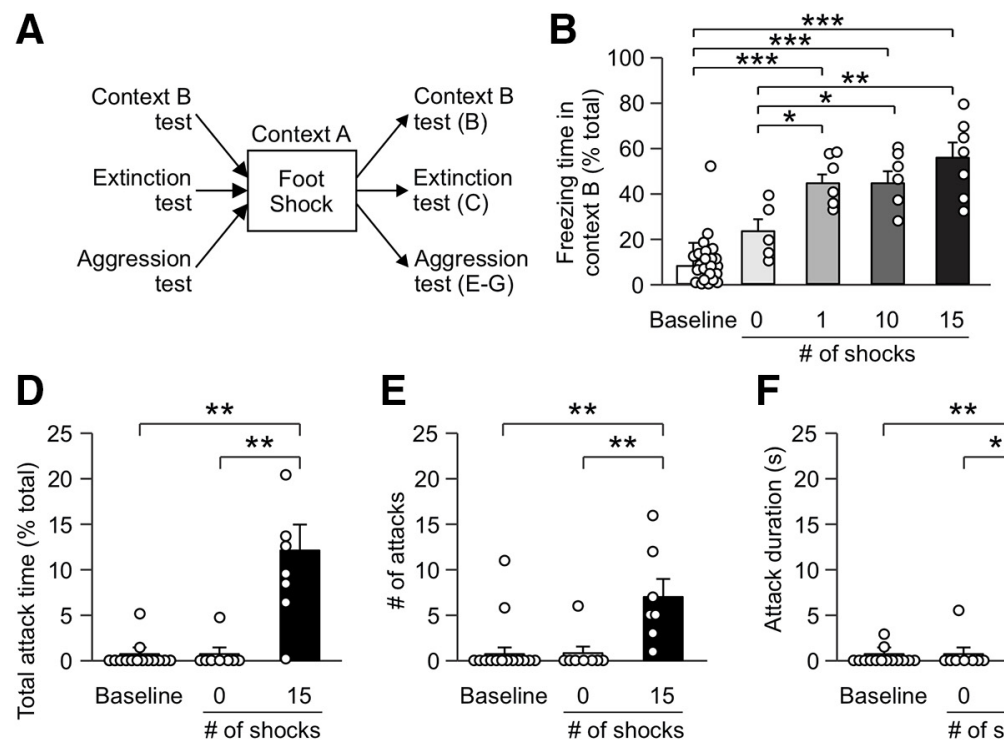

E

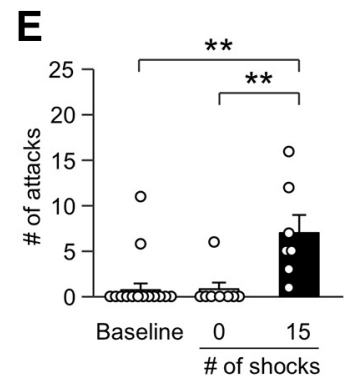

I
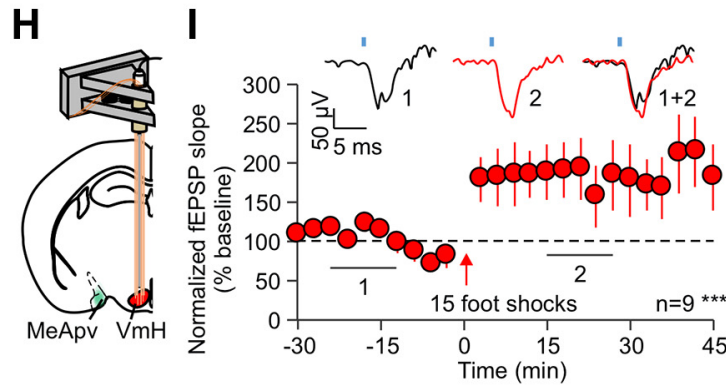

F

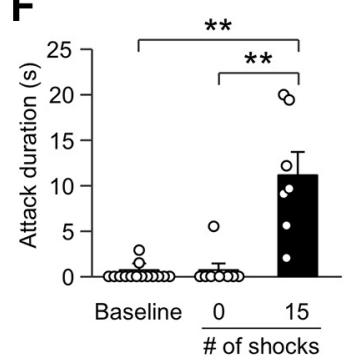

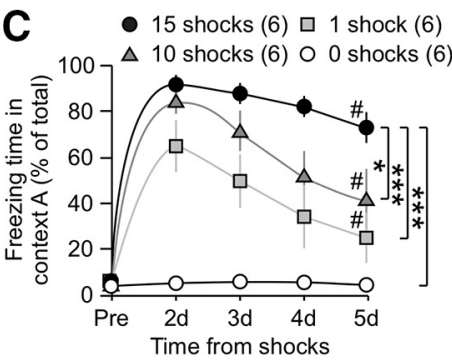

G
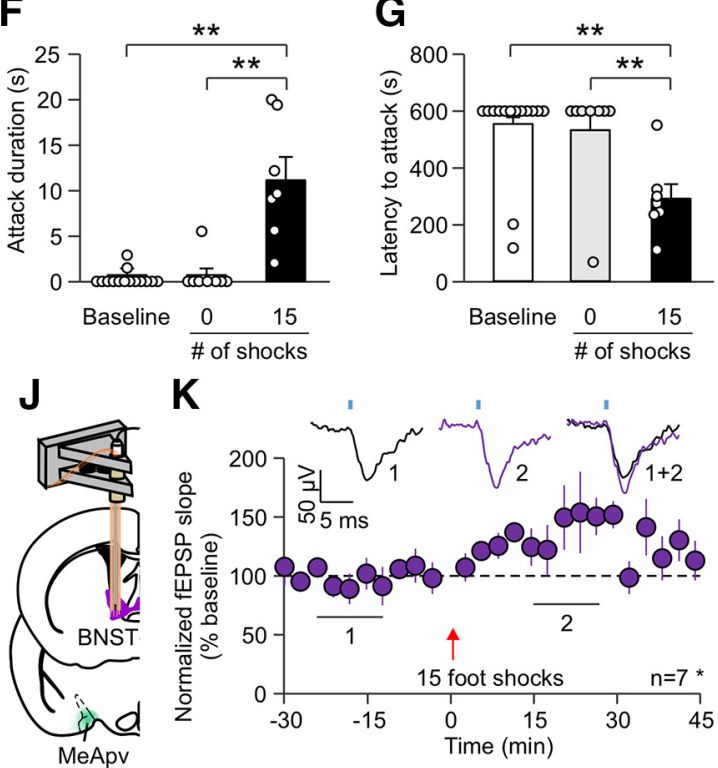

K

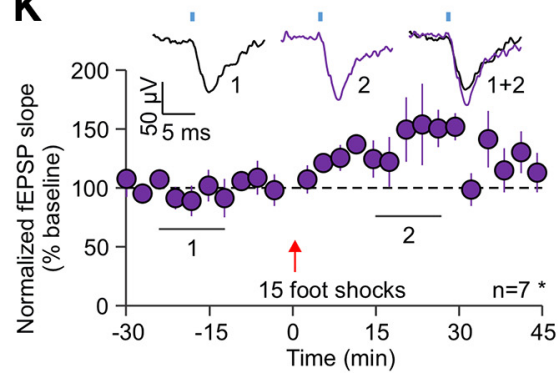

Figure 11. Traumatic stress induces attack behavior and potentiation of MeApv-VmH and MeApv-BNST synapses. $\boldsymbol{A}$, Experimental protocol for behavioral testing after foot shock. Baseline behavior was obtained before foot shock. Separate groups of mice were used for different behavior testing. $\boldsymbol{B}, \boldsymbol{C}$, Fifteen foot shocks enhance fear memory $(\boldsymbol{B})$ and induce long-lasting fear memory (C). B, Analysis of freezing behavior in Context B for mice before foot shock and after receiving no, 1, 10, or 15 foot shocks in Context A followed by 1 foot shock in Context B. Oneway ANOVA was used for statistical analysis across groups: $F_{(4,53)}=41.560, p<0.001, n=24$ (baseline shocks), 5 (0 shocks), 6 (1 shocks), 6 (10 shocks), 7 (15 shocks). Holm-Sidak was used for post hoc multiple comparisons between groups. C, Analysis of freezing behavior across days in Context A for mice before foot shock and after receiving no, 1, 10, or 15 foot shocks in Context A on day 1. One-way ANOVA was used for statistical analysis across days for each condition: $F_{(4,25)}=0.845$ for 0 shocks, 6.292 for 1 shock, 16.325 for 10 shocks, 56.447 for 15 shocks; $p=0.510$ for 0 shocks, $p<0.001$ for 1 shock, $p<0.001$ for 10 shocks, $p<0.001$ for 15 shocks; $n=6$ mice for each condition. One-way ANOVA was used for analysis of group differences on day 5: $F=14.257, p<0.001 .{ }^{\#} p<0.05$, statistical significance within group. ${ }^{*}$ Statistical significance between groups on day 5 . $\mathbf{D}-\mathbf{G}$, Analysis of aggressive behavior for mice receiving no or 15 foot shocks. One-way ANOVA was used for statistical analysis before and after no or 15 foot shocks. $\boldsymbol{H}, \boldsymbol{J}$, Illustration for the viral spread (green areas) and optrode placement (pink bars). $\boldsymbol{I}$, $\boldsymbol{K}$, Normalized slopes of light-evoked fEPSPs recorded at the VmH $(\boldsymbol{I})$ or BNST $(\boldsymbol{K})$ before and after foot shock. Each data point represents the average slope of the late component of 9 evoked fEPSPs. Repeated-measures ANOVA was used for statistical analysis across time points: $F_{(24,200)}=2.622, p<0.001(\mathrm{VmH}) ; F_{(24,150)}=1.638, p=0.04$ (BNST). Two-tailed paired Student's $t$ test was used to identify time points significantly different from baseline. Animal number is indicated in each panel in parentheses. Data are mean \pm SEM. ${ }^{*} p<0.05$. ${ }^{* *} p<0.01$. ${ }^{* * *} p<0.001$.

MeApv projection areas with HFPS or HFPS followed by LFPS. These experiments revealed that the VmH and BNST are critical structures mediating the effects of HFPS on aggression. We then conducted in vivo electrophysiological recordings to test whether

\section{$\leftarrow$}

$F_{(31,279)}=0.907, p=0.613 ;$ HFPS + MK-801, $F_{(30,180)}=0.732, p=0.843$. Two-tailed paired Student's $t$ test was used to identify time points significantly different from baseline. $\boldsymbol{G}, \boldsymbol{H}$, Normalized slopes of light-evoked fEPSPs recorded at the VmH $(\boldsymbol{G})$ or BNST $(\boldsymbol{H})$ before and after the onset of first attack in aggressive mice (AGG), or the onset of first social interaction in nonaggressive mice (NON). LFPS was administered immediately on removal of conspecific. Insets, Representative traces. Each data point represents the average slope of the late component of 9 evoked fEPSPs. Animal number is indicated in each panel in parentheses. Repeated-measures ANOVA was used for statistical analysis across time points under the same experimental condition. For fEPSPs at the VmH: AGG, $F_{(26,351)}=2.830, p<0.001$; NON, $F_{(28,319)}=0.945$, $p=0.548 ;$ AGG + LFPS, $F_{(27,140)}=0.579, p=0.951$. Two-tailed paired Student's $t$ test was used to identify time points significantly different from baseline. For fEPSPs at the BNST: AGG, $F_{(25,286)}=3.555, p<0.001 ; F_{(26,243)}=0.950, p=0.538 ;$ AGG + LFPS, $F_{(25,156)}=0.645$, $p=0.901$. Two-tailed paired Student's $t$ test was used to identify time points significantly different from baseline. Data are mean \pm SEM. ${ }^{*} p<0.05,{ }^{* *} p<0.01,{ }^{* * *} p<0.001$.
HFPS induces synaptic potentiation in these areas. Our results showed that the MeApv-VmH and MeApv-BNST synapses are indeed potentiated by HFPS at time scales similar to those observed after a natural agonistic encounter, and that the synaptic potentiation is suppressed by LFPS and MK-801. Hence, the $\mathrm{MeApv-VmH}$ and MeApv-BNST synapses are candidates of potentiating synapses for "priming." We then used in vivo electrophysiological recording to analyze the strength of MeApv-VmH and MeApv-BNST synapses before and after fighting and found that these synapses are potentiated by attack behavior and that LFPS can abolish this synaptic potentiation. It is noted that LFPS at the MeApv can also suppress naturally occurring aggression. These mice may have been primed by prior attacking experience and therefore susceptible to the LFPS effect. It cannot be ruled out, though, that the MeApv affects aggression generally in addition to primed aggression. Future studies shall address these concerns.

In all, these findings demonstrate that synaptic potentiation in the MeApv aggression circuit mediates aggression priming and identify the specific synapses that are potentiated by priming. 
A

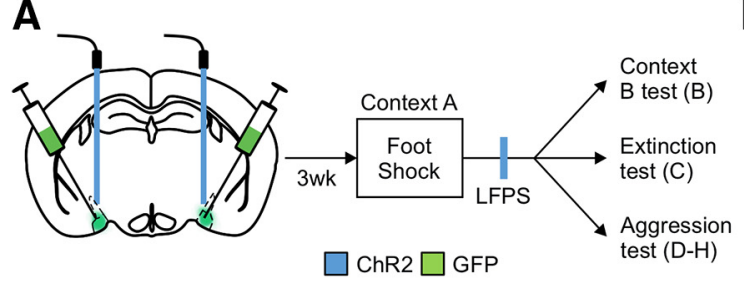

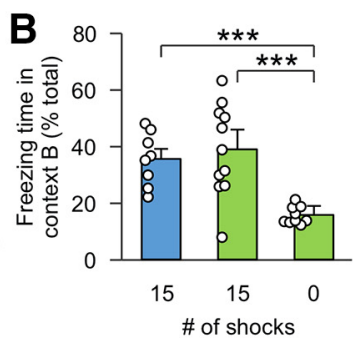
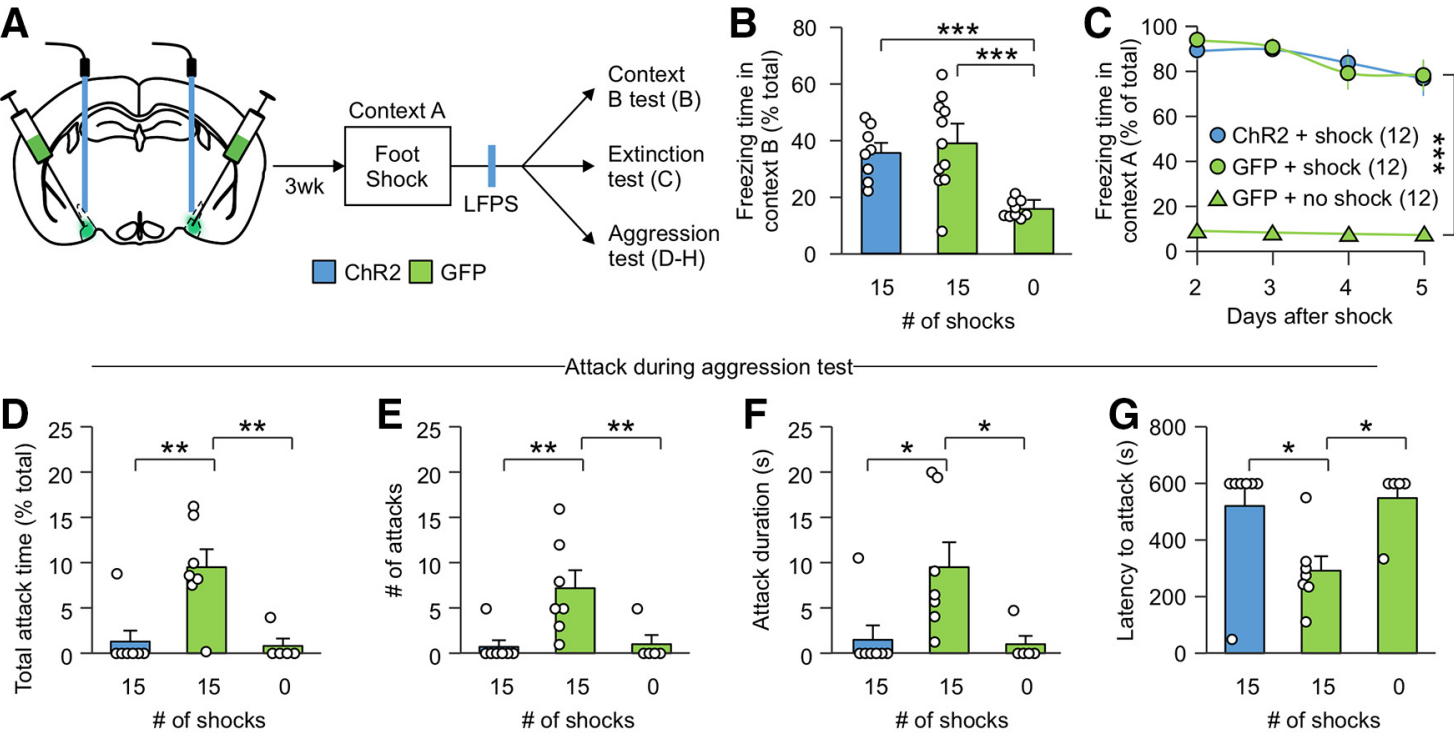

SI during aggression test
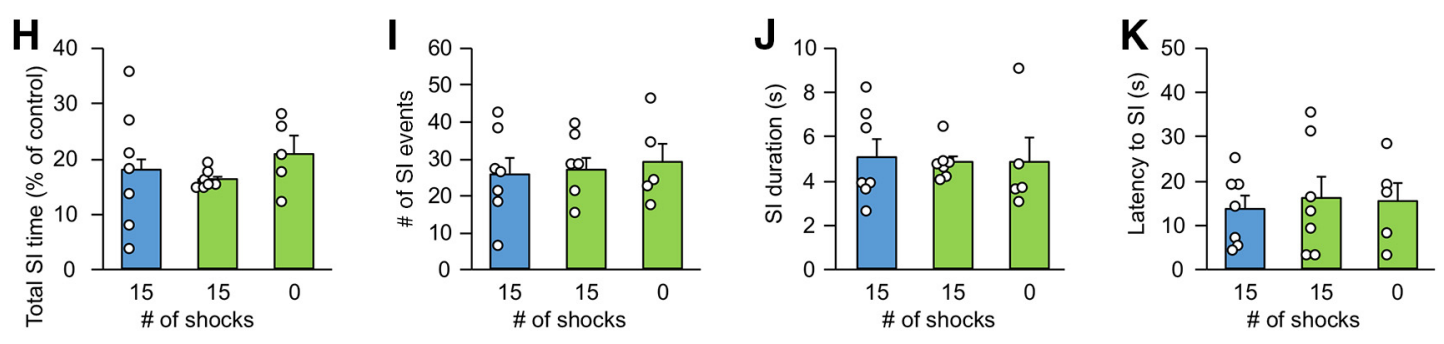

Sociability and locomotion
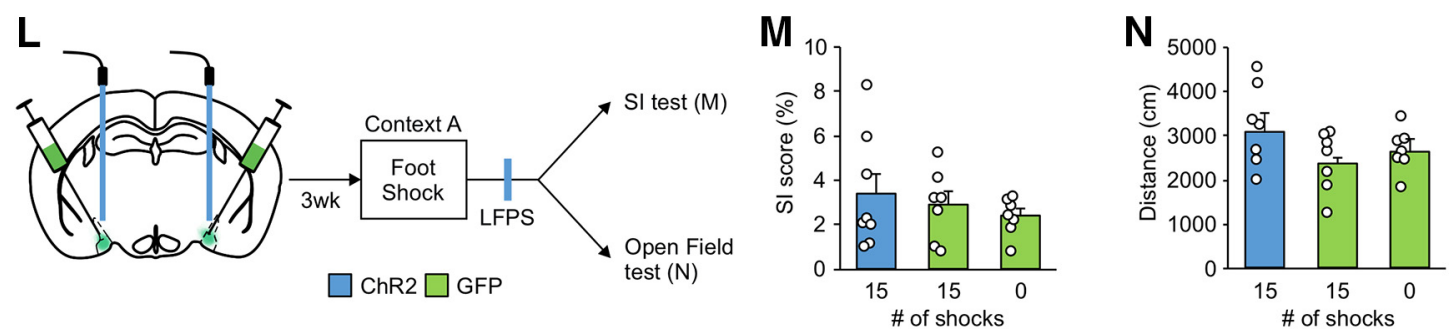

Figure 12. LFPS ameliorates traumatic stress-induced aggression elevation. $\boldsymbol{A}$, Experimental schedule for aggression testing. Left, The viral spread (green areas) and optic fiber placement (blue sticks). Mice were delivered LFPS $10 \mathrm{~min}$ after removal from fear conditioning box. Controls were left in the fear conditioning box for same amount of time without foot shocks. Separate groups of mice were used for different behavior testing. $\boldsymbol{B}$, Freezing behavior in Context $B$ ( $n=8$ ChR2 + shocks, 11 GFP + shocks, 9 GFP mice). Two-way ANOVA was used for statistical analysis across groups in Context $B$ test: $F_{(1,25)}=0.379 ; p=0.543$ (ChR2 vs GFP); $F_{(1,25)}=19.721, p<0.001$ (15 vs no shock). Holm-Sidak was used for post hoc multiple comparisons between two groups. C, Extinction of fear memory in Context A. One-way ANOVA was used for statistical analysis of freezing behavior across days: $F_{(3,44)}=1.199, p=0.321$, for $C h R 2+$ shock; $F_{(3,44)}=2.190, p=0.103$, for GFP + shock; $F_{(3,44)}=1.928, p=0.139$, for GFP + no shock. Two-way ANOVA was used for statistical analysis across groups $(n=12$ ChR2 + shocks, 12 GFP + shocks, 12 GFP mice): $F_{(1,33)}=0.0339, p=0.855$ (ChR2 vs GFP); $F_{(1,33)}=69.725, p<0.001$ (15 vs no shock); Holm-Sidak was used for post hoc multiple comparisons between two groups on day 5. $\boldsymbol{D}-\boldsymbol{K}$, Analysis of attack $(\boldsymbol{D}-\boldsymbol{G})$ and nonaggressive social behavior $(\boldsymbol{H}-\boldsymbol{K})$ during the aggression test for mice receiving LFPS to the MeApv after foot shocks $(n=7$ ChR2 + shocks, 7 GFP + shocks, 5 GFP mice). Two-way ANOVA was used for statistical analysis across groups: $(\boldsymbol{D})$ for virus, $F_{(1,16)}=13.908, p=0.002 ;$ for shocks, $F_{(1,16)}=14.956, p=0.001 ;(\boldsymbol{E})$ for virus, $F_{(1,16)}=8.455, p=0.01$; for shocks, $F_{(1,16)}=11.111, p=0.004 ;(\boldsymbol{F})$ for virus, $F_{(1,16)}=7.599, p=0.014$; for shocks, $F_{(1,16)}=7.968, p=0.012 ;(\boldsymbol{G})$ for virus, $F_{(1,16)}=7.203, p=0.016 ;$ for shocks, $F_{(1,16)}=6.986, p=0.018$. $(\boldsymbol{H})$ for virus, $F_{(1,16)}=0.238, p=0.632$; for shocks, $F_{(1,16)}=1.108, p=0.308 ;(\boldsymbol{I})$ for virus, $F_{(1,16)}=0.0494, p=0.827 ;$ for shocks, $F_{(1,16)}=0.089, p=0.770 ;(J)$ for virus, $F_{(1,16)}=0.072, p=0.792$; for shocks, $F_{(1,16)}=0.0002, p=0.989 ;(K)$ for virus, $F_{(1,16)}=0.188, p=0.671$; for shocks, $F_{(1,16)}=0.014, p=0.909$. Holm-Sidak was used for post hoc multiple comparisons between two groups. $\boldsymbol{L}$, Experimental schedule for behavioral testing after foot shock. Left, The viral spread (green areas) and optic fiber placement (blue sticks). $\boldsymbol{M}, \boldsymbol{N}$, Analysis of sociability $(\boldsymbol{M})$ and distance traveled in the open field test $(\boldsymbol{N})$ after receiving no or 15 foot shocks, followed by LFPS. Two-way ANOVA was used for statistical analysis across groups: ( $\boldsymbol{M})$ for virus, $F_{(1,19)}=0.247, p=0.625$; for shocks, $F_{(1,19)}=0.293, p=0.594 ;(\mathcal{N})$ for virus, $F_{(1,18)}=4.285, p=0.053 ;$ for shocks, $F_{(1,18)}=0.462, p=0.505 ; S I$ during aggression test, $n=8$ (ChR2 + shocks), 7 (GFP + shocks), 7 (GFP) mice; open field, $n=7$ (ChR2 + shocks), 7 (GFP + shocks), 6 (GFP) mice. Holm-Sidak was used for post hoc analysis of multiple comparisons between two groups. Data are mean \pm SEM. ${ }^{*} p<0.05,{ }^{* *} p<0.01,{ }^{* * *} p<0.001$.

\section{$\mathrm{VmH}$ and BNST regulate different stages of aggressive behavior}

Both the VmH and BNST receive projections from the MeApv and are activated in dominant mice after attacking a conspecific, as shown by c-Fos labeling (Kollack-Walker and Newman, 1995;
Gobrogge et al., 2007; Lin et al., 2011). The VmH is a critical subnucleus within the hypothalamic attack area in rodents (Kruk et al., 1983; Lammers et al., 1988). Electrical and optogenetic stimulation of the VmH can elicit attack behavior (Kruk et al., 1983; Lammers et al., 1988; Lin et al., 2011). Electrical stimulation of 
the BNST can modulate attack behavior in cats (Shaikh et al., 1986), while blocking AMPA or metabotropic glutamate receptor Type 7 receptors in the BNST reduces aggression (MasugiTokita et al., 2016). Optogenetic stimulation of projections from MeA neurons expressing the neuropeptide Y receptor type 1 to the BNST increases nudging activity as opposed to aggression (Padilla et al., 2016). Despite the evidence supporting the involvement of the $\mathrm{VmH}$ and BNST in aggressive behavior, it is unclear whether the $\mathrm{VmH}$ and BNST play redundant or distinct roles in aggression.

Our study indicates that the MeApv projections to the $\mathrm{VmH}$ and BNST drive different stages of aggressive behavior. The functional divergence of MeApv inputs to the $\mathrm{VmH}$ and BNST is supported by recent findings that these efferent pathways have dissociable effects on conflict approach and avoidance behavior (Miller et al., 2019). Here, we show that selective potentiation of the MeApv to $\mathrm{VmH}$ projection primarily prolongs individual attacks and inhibiting this pathway during HFPS of the MeApv blocks the concomitant increase in attack duration while preserving the overall number of initiated attacks. By contrast, selective potentiation of the MeApv to BNST projection increases the number of attacks with no effect on the duration of individual attacks. Although potentiating the MeApv-BNST pathway alone is not sufficient to prolong attacks, the BNST needs to be potentiated for HFPS of the MeApv to prolong attack, as shown by the finding that BNST inactivation during MeApv HFPS obliterates its effect on attack duration.

Hence, it appears that MeApv-driven aggression priming relies on potentiation of the MeApv-BNST pathway for attack initiation and the MeApv-VmH pathway to sustain an already occurring attack. It is perceivable that parsing a single attacking event into steps and regulating them through separate pathways allows for more precise temporal control of attack behavior. Moreover, it is possible that different stages of attack can be adjusted separately according to social contexts and emotional valence by integrating non-MeApv afferents to the $\mathrm{VmH}$ and BNST. Future studies will be aimed at examining how the MeApv circuit regulates other stages of attack behavior during primed aggression.

\section{Traumatic stress-induced aggression}

In addition to attack priming, our study shows that traumatic stress potentiates $\mathrm{MeApv}-\mathrm{VmH}$ and $\mathrm{MeApv}-\mathrm{BNST}$ pathways, leading to traumatic experience-induced aggression increase. A serious consequence of traumatic stress is PTSD, which has a lifetime prevalence of $\sim 6 \%-8 \%$ (Kessler et al., 2005). It can be disabling and is characterized by persistent reexperiencing of traumatic events and hyperarousal symptoms, such as recurrent angry and aggressive outbursts (McHugh et al., 2012; Smerin et al., 2016). The mechanism for persistent and intrusive memories after traumatic events is emerging from clinical and animal studies (Desmedt et al., 2015). For example, hyperactivity of the amygdala has been implicated (Koenigs and Grafman, 2009; Stevens et al., 2017). Despite the severe, destructive social problems associated with unchecked aggression, little is known about the underlying neural substrate.

Our study shows that traumatic stress can potentiate synaptic transmission between the MeApv and its synaptic partners, the $\mathrm{VmH}$ and BNST, and that weakening synaptic transmission in MeApv-mediated aggression pathways prevents traumatic stressinduced increased aggression. The MeApv-VmH and MeApvBNST synapses and signaling pathways underlying synaptic potentiation, therefore, may be potentially targeted to develop therapies to alleviate aggression symptoms associated with psychiatric disorders, such as PTSD. Intriguing possibilities include the use of deep brain stimulation to alter synaptic strength within MeApv aggression circuits in patients with PTSD who are resistant to pharmacotherapy or psychotherapy. It is noted that deep brain stimulation has been administered to the basolateral amygdala of a patient with severe PTSD to reduce nightmares (Langevin et al., 2016). Our findings suggest that the MeApv could be a target of deep brain stimulation to treat the aggression symptom associated with PTSD.

In conclusion, our study reveals that potentiation of synapses between the MeApv and the $\mathrm{VmH}$ and BNST leads to increased aggression. This process mediates increased aggression following fighting and traumatic experience. Synaptic depression of these MeApv-mediated aggression circuits, conversely, dampens primed aggression and prevents traumatic stress-induced aggression elevation. This study, therefore, identifies key synaptic mechanisms and neural circuits for experience-dependent modification of aggressive behavior.

\section{References}

American Psychiatric Association (2013) Diagnostic and statistical manual of mental disorders, Ed 5. Washington, DC: American Psychiatric Association.

Bagot RC, Parise EM, Peña CJ, Zhang HX, Maze I, Chaudhury D, Persaud B, Cachope R, Bolaños-Guzmán CA, Cheer JF, Cheer J, Deisseroth K, Han MH, Nestler EJ (2015) Ventral hippocampal afferents to the nucleus accumbens regulate susceptibility to depression. Nat Commun 6:7062.

Bayless DW, Yang T, Mason MM, Susanto AA, Lobdell A, Shah NM (2019) Limbic neurons shape sex recognition and social behavior in sexually naive males. Cell 176:1190-1205.e1120.

Benson DL, Isackson PJ, Gall CM, Jones EG (1992) Contrasting patterns in the localization of glutamic-acid decarboxylase and $\mathrm{Ca}^{2+} /$ calmodulin protein-kinase gene-expression in the rat central nervous system. Neuroscience 46:825-849.

Blanchard RJ, Blanchard DC (1977) Aggressive behavior in the rat. Behav Biol 21:197-224.

Blanchard DC, Blanchard RJ (2003a) What can animal aggression research tell us about human aggression? Horm Behav 44:171-177.

Blanchard RJ, Blanchard DC (2003b) Bringing natural behaviors into the laboratory: a tribute to Paul MacLean. Physiol Behav 79:515-524.

Blanchard RJ, O'Donnell V, Blanchard DC (1979) Attack and defensive behaviors in the albino mouse. Aggr Behav 5:341-352.

Bliss TV, Collingridge GL (1993) A synaptic model of memory: long-term potentiation in the hippocampus. Nature 361:31-39.

Butler RK, Sharko AC, Oliver EM, Brito-Vargas P, Kaigler KF, Fadel JR, Wilson MA (2011) Activation of phenotypically-distinct neuronal subpopulations of the rat amygdala following exposure to predator odor. Neuroscience 175:133-144.

Canteras NS, Simerly RB, Swanson LW (1995) Organization of projections from the medial nucleus of the amygdala: a PHAL study in the rat. J Comp Neurol 360:213-245.

Carrillo M, Ricci LA, Melloni RH (2011) Glutamate-vasopressin interactions and the neurobiology of anabolic steroid-induced offensive aggression. Neuroscience 185:85-96.

Chang CH, Gean PW (2019) The ventral hippocampus controls stress-provoked impulsive aggression through the ventromedial hypothalamus in post-weaning social isolation mice. Cell Rep 28:1195-1205.e1193.

Chang CH, Hsiao YH, Chen YW, Yu YJ, Gean PW (2015) Social isolationinduced increase in NMDA receptors in the hippocampus exacerbates emotional dysregulation in mice. Hippocampus 25:474-485.

Chang CH, Su CL, Gean PW (2018) Mechanism underlying NMDA blockade-induced inhibition of aggression in post-weaning socially isolated mice. Neuropharmacology 143:95-105.

Chen WR, Lee SH, Kato K, Spencer DD, Shepherd GM, Williamson A (1996a) Long-term modifications of synaptic efficacy in the human inferior and middle temporal cortex. Proc Natl Acad Sci USA 93:8011-8015. 
Chen WR, Lee S, Kato K, Spencer DD, Shepherd GM, Williamson A (1996b) Long-term modifications of synaptic efficacy in the human inferior and middle temporal cortex. Proc Natl Acad Sci USA 93:8011-8015.

Cheong RY, Czieselsky K, Porteous R, Herbison AE (2015) Expression of ESR1 in glutamatergic and GABAergic neurons is essential for normal puberty onset, estrogen feedback, and fertility in female mice. J Neurosci 35:14533-14543.

Choi GB, Dong HW, Murphy AJ, Valenzuela DM, Yancopoulos GD, Swanson LW, Anderson DJ (2005) Lhx6 delineates a pathway mediating innate reproductive behaviors from the amygdala to the hypothalamus. Neuron 46:647-660.

Coolen LM, Wood RI (1998) Bidirectional connections of the medial amygdaloid nucleus in the Syrian hamster brain: simultaneous anterograde and retrograde tract tracing. J Comp Neurol 399:189-209.

de Boer SF, van der Vegt BJ, Koolhaas JM (2003) Individual variation in aggression of feral rodent strains: a standard for the genetics of aggression and violence? Behav Genet 33:485-501.

Desmedt A, Marighetto A, Piazza PV (2015) Abnormal Fear Memory as a Model for Posttraumatic Stress Disorder. Biological Psychiatry 78:290297.

Erskine MS (1993) Mating-induced increases in FOS protein in preoptic area and medial amygdala of cycling female rats. Brain Res Bull 32:447-451.

Falkner AL, Dollar P, Perona P, Anderson DJ, Lin D (2014) Decoding ventromedial hypothalamic neural activity during male mouse aggression. J Neurosci 34:5971-5984.

Fernandez-Fewell GD, Meredith M (1994) c-fos expression in vomeronasal pathways of mated or pheromone-stimulated male golden hamsters: contributions from vomeronasal sensory input and expression related to mating performance. J Neurosci 14:3643-3654.

Flannelly KJ, Blanchard RJ, Muraoka MY, Flannelly L (1982) Copulation increases offensive attack in male rats. Physiol Behav 29:381-385.

Fuxjager MJ, Forbes-Lorman RM, Coss DJ, Auger CJ, Auger AP, Marler CA (2010) Winning territorial disputes selectively enhances androgen sensitivity in neural pathways related to motivation and social aggression. Proc Natl Acad Sci USA 107:12393-12398.

Gobrogge KL, Liu Y, Jia X, Wang Z (2007) Anterior hypothalamic neural activation and neurochemical associations with aggression in pairbonded male prairie voles. J Comp Neurol 502:1109-1122.

Golden SA, Heshmati M, Flanigan M, Christoffel DJ, Guise K, Pfau ML, Aleyasin H, Menard C, Zhang H, Hodes GE, Bregman D, Khibnik L, Tai J, Rebusi N, Krawitz B, Chaudhury D, Walsh JJ, Han MH, Shapiro ML, Russo SJ (2016) Basal forebrain projections to the lateral habenula modulate aggression reward. Nature 534:688-692.

Golding NL, Staff NP, Spruston N (2002) Dendritic spikes as a mechanism for cooperative long-term potentiation. Nature 418:326-331.

Gomez DM, Newman SW (1992) Differential projections of the anterior and posterior regions of the medial amygdaloid nucleus in the Syrian hamster. J Comp Neurol 317:195-218.

Grover LM, Kim E, Cooke JD, Holmes WR (2009) LTP in hippocampal area CA1 is induced by burst stimulation over a broad frequency range centered around delta. Learn Mem 16:69-81.

Haller J, Toth M, Halasz J, De Boer SF (2006) Patterns of violent aggressioninduced brain c-fos expression in male mice selected for aggressiveness. Physiol Behav 88:173-182.

Hashikawa K, Hashikawa Y, Falkner A, Lin D (2016) The neural circuits of mating and fighting in male mice. Curr Opin Neurobiol 38:27-37.

Heeb MM, Yahr P (1996) c-Fos immunoreactivity in the sexually dimorphic area of the hypothalamus and related brain regions of male gerbils after exposure to sex-related stimuli or performance of specific sexual behaviors. Neuroscience 72:1049-1071.

Hong W, Kim DW, Anderson DJ (2014) Antagonistic control of social vs repetitive self-grooming behaviors by separable amygdala neuronal subsets. Cell 158:1348-1361.

Hoopfer ED (2016) Neural control of aggression in Drosophila. Curr Opin Neurobiol 38:109-118.

Hsu Y, Earley RL, Wolf LL (2006) Modulation of aggressive behaviour by fighting experience: mechanisms and contest outcomes. Biol Rev Camb Philos Soc 81:33-74.

Ishii KK, Osakada T, Mori H, Miyasaka N, Yoshihara Y, Miyamichi K, Touhara K (2017) A labeled-line neural circuit for pheromone-mediated sexual behaviors in mice. Neuron 95:123-137.e8.
Jiao Y, Sun Z, Lee T, Fusco FR, Kimble TD, Meade CA, Cuthbertson S, Reiner A (1999) A simple and sensitive antigen retrieval method for freefloating and slide-mounted tissue sections. J Neurosci Methods 93:149162.

Jones EG, Huntley GW, Benson DL (1994) Alpha calcium/calmodulin-dependent protein kinase II selectively expressed in a subpopulation of excitatory neurons in monkey sensory-motor cortex: comparison with GAD67 expression. J Neurosci 14:611-629.

Kaidanovich-Beilin O, Lipina T, Vukobradovic I, Roder J, Woodgett JR (2011) Assessment of social interaction behaviors. J Vis Exp 48:2473.

Keshavarzi S, Sullivan RK, Ianno DJ, Sah P (2014) Functional properties and projections of neurons in the medial amygdala. J Neurosci 34:8699-8715.

Kessler RC, Berglund P, Demler O, Jin R, Merikangas KR, Walters EE (2005) Lifetime prevalence and age-of-onset distributions of DSM-IV disorders in the National Comorbidity Survey Replication. Arch Gen Psychiatry 62:593-602.

Kim WB, Cho JH (2017) Encoding of discriminative fear memory by inputspecific LTP in the amygdala. Neuron 95:1129-1146.e1125.

Kim Y, Venkataraju KU, Pradhan K, Mende C, Taranda J, Turaga SC, Arganda-Carreras I, Ng L, Hawrylycz MJ, Rockland KS, Seung HS, Osten P (2015) Mapping social behavior-induced brain activation at cellular resolution in the mouse. Cell Rep 10:292-305.

Koenigs M, Grafman J (2009) Posttraumatic stress disorder: the role of medial prefrontal cortex and amygdala. Neuroscientist 15:540-548.

Kollack-Walker S, Newman SW (1995) Mating and agonistic behavior produce different patterns of Fos immunolabeling in the male Syrian hamster brain. Neuroscience 66:721-736.

Kollack-Walker S, Newman SW (1997) Mating-induced expression of c-fos in the male Syrian hamster brain: role of experience, pheromones, and ejaculations. J Neurobiol 32:481-501.

Koolhaas JM, Coppens CM, de Boer SF, Buwalda B, Meerlo P, Timmermans PJ (2013) The resident-intruder paradigm: a standardized test for aggression, violence and social stress. J Vis Exp 77:e4367.

Kruk MR (2014) Hypothalamic attack: a wonderful artifact or a useful perspective on escalation and pathology in aggression? A viewpoint. Curr Top Behav Neurosci 17:143-188.

Kruk MR, Van der Poel AM, Meelis W, Hermans J, Mostert PG, Mos J, Lohman AH (1983) Discriminant analysis of the localization of aggression-inducing electrode placements in the hypothalamus of male rats. Brain Res 260:61-79.

Lammers JH, Kruk MR, Meelis W, van der Poel AM (1988) Hypothalamic substrates for brain stimulation-induced attack, teeth-chattering and social grooming in the rat. Brain Res 449:311-327.

Langevin JP, Chen JW, Koek RJ, Sultzer DL, Mandelkern MA, Schwartz HN, Krahl SE (2016) Deep brain stimulation of the basolateral amygdala: targeting technique and electrodiagnostic findings. Brain Sci 6:28.

Largerspetz K (1969) Aggression and aggressiveness in laboratory mice. New York: Wiley.

Lee H, Kim DW, Remedios R, Anthony TE, Chang A, Madisen L, Zeng H, Anderson DJ (2014) Scalable control of mounting and attack by Esr $1^{+}$ neurons in the ventromedial hypothalamus. Nature 509:627-632.

Lin D, Boyle MP, Dollar P, Lee H, Lein ES, Perona P, Anderson DJ (2011) Functional identification of an aggression locus in the mouse hypothalamus. Nature 470:221-226.

Liu XB, Jones EG (1996) Localization of alpha type II calcium calmodulin-dependent protein kinase at glutamatergic but not gamma-aminobutyric acid (GABAergic) synapses in thalamus and cerebral cortex. Proc Natl Acad Sci USA 93:7332-7336.

Mackintosh JH, Grant EC (1963) A comparison of the social postures of some common laboratory rodents. Behavior 21:246-259.

Masugi-Tokita M, Flor PJ, Kawata M (2016) Metabotropic glutamate receptor subtype 7 in the bed nucleus of the stria terminalis is essential for intermale aggression. Neuropsychopharmacology 41:726-735.

McHugh T, Forbes D, Bates G, Hopwood M, Creamer M (2012) Anger in PTSD: Is there a need for a concept of PTSD-related posttraumatic anger? Clinical Psychology Review 32:93-104

Miczek KA, O'Donnell JM (1978) Intruder-evoked aggression in isolated and nonisolated mice: effects of psychomotor stimulants and L-dopa. Psychopharmacology (Berl) 57:47-55.

Miczek KA, Maxson SC, Fish EW, Faccidomo S (2001) Aggressive behavioral phenotypes in mice. Behav Brain Res 125:167-181. 
Miczek KA, de Boer SF, Haller J (2013) Excessive aggression as model of violence: a critical evaluation of current preclinical methods. Psychopharmacology (Berl) 226:445-458

Miller SM, Marcotulli D, Shen A, Zweifel LS (2019) Divergent medial amygdala projections regulate approach-avoidance conflict behavior. Nat Neurosci 22:565-575.

Morgan JI, Cohen DR, Hempstead JL, Curran T (1987) Mapping patterns of c-fos expression in the central nervous system after seizure. Science 237:192-197.

Mpakopoulou M, Gatos H, Brotis A, Paterakis KN, Fountas KN (2008) Stereotactic amygdalotomy in the management of severe aggressive behavioral disorders. Neurosurg Focus 25:E6.

Nabavi S, Fox R, Proulx CD, Lin JY, Tsien RY, Malinow R (2014) Engineering a memory with LTD and LTP. Nature 511:348-352.

Nathanson JL, Yanagawa Y, Obata K, Callaway EM (2009) Preferential labeling of inhibitory and excitatory cortical neurons by endogenous tropism of adeno-associated virus and lentivirus vectors. Neuroscience 161:441450 .

Nelson RJ, Trainor BC (2007) Neural mechanisms of aggression. Nat Rev Neurosci 8:536-546.

Nguyen PV, Kandel ER (1997) Brief theta-burst stimulation induces a transcription-dependent late phase of LTP requiring cAMP in area CA1 of the mouse hippocampus. Learn Mem 4:230-243.

Olivier B (1981) Selective anti-aggressive properties of Du-27725: ethological analyses of intermale and territorial aggression in the male rat. Pharmacol Biochem Behav 14:61-77.

Olivier B, van Dalen D (1982) Social behaviour in rats and mice: an ethologically based model for differentiating psychoactive drugs. Aggr Behav $8: 163-168$

Olson VG, Rockett HR, Reh RK, Redila VA, Tran PM, Venkov HA, Defino MC, Hague C, Peskind ER, Szot P, Raskind MA (2011) The role of norepinephrine in differential response to stress in an animal model of posttraumatic stress disorder. Biol Psychiatry 70:441-448.

Padilla SL, Qiu J, Soden ME, Sanz E, Nestor CC, Barker FD, Quintana A, Zweifel LS, Ronnekleiv OK, Kelly MJ, Palmiter RD (2016) Agouti-related peptide neural circuits mediate adaptive behaviors in the starved state. Nat Neurosci 19:734-741.

Pan Y, Zhu Q, Xu T, Zhang Z, Wang Z (2020) Aggressive behavior and brain neuronal activation in sexually naive male Mongolian gerbils. Behav Brain Res 378:112276.

Pardo-Bellver C, Cádiz-Moretti B, Novejarque A, Martínez-García F, Lanuza E (2012) Differential efferent projections of the anterior, posteroventral, and posterodorsal subdivisions of the medial amygdala in mice. Front Neuroanat 6:33.

Potegal M (1992) Time course of aggressive arousal in female hamsters and male rats. Behav Neural Biol 58:120-124.

Potegal M, Popken J (1985) The time course of attack priming effects in female golden hamsters. Behav Processes 11:199-208.

Potegal M, Hebert M, DeCoster M, Meyerhoff JL (1996a) Brief, high-frequency stimulation of the corticomedial amygdala induces a delayed and prolonged increase of aggressiveness in male Syrian golden hamsters. Behav Neurosci 110:401-412.

Potegal M, Ferris CF, Hebert M, Meyerhoff J, Skaredoff L (1996b) Attack priming in female Syrian golden hamsters is associated with a c-fos-coupled process within the corticomedial amygdala. Neuroscience 75:869-880.

Pynoos RS, Ritzmann RF, Steinberg AM, Goenjian A, Prisecaru I (1996) A behavioral animal model of posttraumatic stress disorder featuring repeated exposure to situational reminders. Biol Psychiatry 39:129-134.

Rau V, DeCola JP, Fanselow MS (2005) Stress-induced enhancement of fear learning: an animal model of posttraumatic stress disorder. Neurosci Biobehav Rev 29:1207-1223.

Remedios R, Kennedy A, Zelikowsky M, Grewe BF, Schnitzer MJ, Anderson DJ (2017) Social behaviour shapes hypothalamic neural ensemble representations of conspecific sex. Nature 550:388-392.

Remy S, Spruston N (2007) Dendritic spikes induce single-burst long-term potentiation. Proc Natl Acad Sci USA 104:17192-17197.

Roubertoux PL, Roy IL Mortaud S, Perez-Diaz F, Tordjman S (1999) Measuring aggression in the mouse. In: Techniques in the behavioral and neural sciences. Amsterdam: Elsevier.
Shaikh MB, Brutus M, Siegel HE, Siegel A (1986) Regulation of feline aggression by the bed nucleus of stria terminalis. Brain Res Bull 16:179-182.

Shindou T, Watanabe S, Yamamoto K, Nakanishi H (1993) NMDA receptordependent formation of long-term potentiation in the rat medial amygdala neuron in an in vitro slice preparation. Brain Res Bull 31:667-672.

Silva BA, Mattucci C, Krzywkowski P, Murana E, Illarionova A, Grinevich V, Canteras NS, Ragozzino D, Gross CT (2013) Independent hypothalamic circuits for social and predator fear. Nat Neurosci 16:1731-1733.

Silverman JL, Yang M, Lord C, Crawley JN (2010) Behavioural phenotyping assays for mouse models of autism. Nat Rev Neurosci 11:490-502.

Simon NG, Gandelman R (1981) Threat postures signal impending attack in mice. Behav Neural Biol 33:509-513.

Smerin S, Chen A, Li H (2016) Neurophysiology of aggression in posttraumatic stress disorder. Journal of Psychiatry 19:1-11.

Stevens JS, Kim YJ, Galatzer-Levy IR, Reddy R, Ely TD, Nemeroff CB, Hudak LA, Jovanovic T, Rothbaum BO, Ressler KJ (2017) Amygdala Reactivity and Anterior Cingulate Habituation Predict Posttraumatic Stress Disorder Symptom Maintenance After Acute Civilian Trauma. Biol Psychiatry 81:1023-1029.

Stevenson PA, Rillich J (2012) The decision to fight or flee: insights into underlying mechanism in crickets. Front Neurosci 6:118.

Taft CT, Creech SK, Kachadourian L (2012) Assessment and treatment of posttraumatic anger and aggression: a review. J Rehabil Res Dev 49:777788 .

Taft CT, Creech SK, Murphy CM (2017) Anger and aggression in PTSD. Curr Opin Psychol 14:67-71.

Tellegen A, Horn JM (1972) Primary aggressive motivation in three inbred strains of mice. J Comp Physiol Psychol 78:297-304.

Unger EK, Burke KJ Jr, Yang CF, Bender KJ, Fuller PM, Shah NM (2015) Medial amygdalar aromatase neurons regulate aggression in both sexes. Cell Rep 10:453-462.

Valzelli L (1973) The "isolation syndrome" in mice. Psychopharmacologia 31:305-320.

Van Voorhees EE, Dennis PA, Neal LC, Hicks TA, Calhoun PS, Beckham JC, Elbogen EB (2016) Posttraumatic stress disorder, hostile cognitions, and aggression in Iraq/Afghanistan era veterans. Psychiatry 79:70-84.

Wallen-Mackenzie A, Nordenankar K, Fejgin K, Lagerstrom MC, Emilsson L, Fredriksson R, Wass C, Andersson D, Egecioglu E, Andersson M, Strandberg J, Lindhe O, Schioth HB, Chergui K, Hanse E, Langstrom B, Fredriksson A, Svensson L, Roman E, Kullander K (2009) Restricted cortical and amygdaloid removal of vesicular glutamate transporter 2 in preadolescent mice impacts dopaminergic activity and neuronal circuitry of higher brain function. J Neurosci 29:2238-2251.

Wang L, Chen IZ, Lin D (2015) Collateral pathways from the ventromedial hypothalamus mediate defensive behaviors. Neuron 85:1344-1358.

Wang XJ, Zhang CZ, Szabo G, Sun QQ (2013) Distribution of CaMKII alpha expression in the brain in vivo, studied by CaMKII alpha-GFP mice. Brain Res 1518:9-25.

Watanabe Y, Ikegaya Y, Saito H, Abe K (1995) Roles of GABAA, NMDA and muscarinic receptors in induction of long-term potentiation in the medial and lateral amygdala in vitro. Neurosci Res 21:317-322.

Watanabe Y, Ikegaya Y, Saito H, Abe K (1996) Opposite regulation by the beta-adrenoceptor-cyclic AMP system of synaptic plasticity in the medial and lateral amygdala in vitro. Neuroscience 71:1031-1035

Xiong Q, Znamenskiy P, Zador AM (2015) Selective corticostriatal plasticity during acquisition of an auditory discrimination task. Nature 521:348351.

Yang CF, Chiang MC, Gray DC, Prabhakaran M, Alvarado M, Juntti SA, Unger EK, Wells JA, Shah NM (2013) Sexually dimorphic neurons in the ventromedial hypothalamus govern mating in both sexes and aggression in males. Cell 153:896-909.

Yang T, Yang CF, Chizari MD, Maheswaranathan N, Burke KJ Jr, Borius M, Inoue S, Chiang MC, Bender KJ, Ganguli S, Shah NM (2017) Social control of hypothalamus-mediated male aggression. Neuron 95:955-970. e954.

Zhou T, Zhu H, Fan Z, Wang F, Chen Y, Liang H, Yang Z, Zhang L, Lin L, Zhan Y, Wang Z, Hu H (2017) History of winning remodels thalamoPFC circuit to reinforce social dominance. Science 357:162-168.

Zhu Y, Wienecke CF, Nachtrab G, Chen X (2016) A thalamic input to the nucleus accumbens mediates opiate dependence. Nature 530:219-222. 